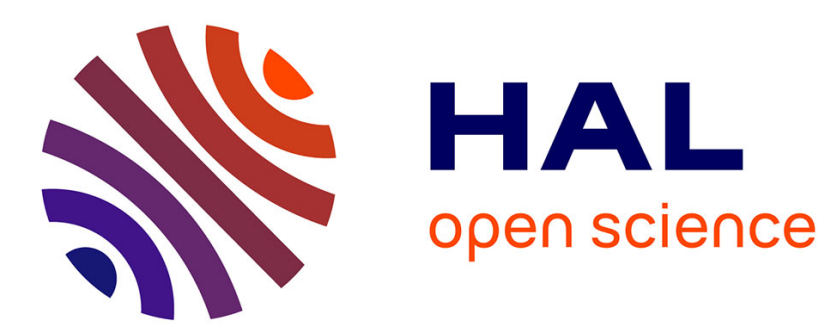

\title{
Quantitative analysis of metastability in reversible diffusion processes via a Witten complex approach: the case with boundary.
}

Francis Nier, Bernard Helffer

\section{- To cite this version:}

Francis Nier, Bernard Helffer. Quantitative analysis of metastability in reversible diffusion processes via a Witten complex approach: the case with boundary.. 2004. hal-00002744

HAL Id: hal-00002744

https://hal.science/hal-00002744

Preprint submitted on 1 Sep 2004

HAL is a multi-disciplinary open access archive for the deposit and dissemination of scientific research documents, whether they are published or not. The documents may come from teaching and research institutions in France or abroad, or from public or private research centers.
L'archive ouverte pluridisciplinaire HAL, est destinée au dépôt et à la diffusion de documents scientifiques de niveau recherche, publiés ou non, émanant des établissements d'enseignement et de recherche français ou étrangers, des laboratoires publics ou privés. 


\title{
Quantitative analysis of metastability in reversible diffusion processes via a Witten complex approach: the case with boundary
}

\author{
Bernard Helffer* and Francis Nier ${ }^{\dagger}$
}

August 30, 2004

\begin{abstract}
This article is a continuation of previous works by Bovier-EckhoffGayrard-Klein, Bovier-Gayrard-Klein and Helffer-Klein-Nier. The main object is the analysis of the small eigenvalues (as $h \rightarrow 0$ ) of the Laplacian attached to the quadratic form

$$
C_{0}^{\infty}(\Omega) \ni v \mapsto h^{2} \int_{\Omega}|\nabla v(x)|^{2} e^{-2 f(x) / h} d x
$$

where $\Omega$ is a bounded connected open set with $C^{\infty}$-boundary and $f$ is a Morse function on $M=\bar{\Omega}$. The previous works were devoted to the case of a manifold $M$ which is compact but without boundary or $\mathbb{R}^{n}$. Our aim is here to analyze the case with boundary. After the introduction of a Witten cohomology complex adapted to the case with boundary, we give a very accurate asymptotics for the exponentially small eigenvalues. In particular, we analyze the effect of the boundary in the asymptotics.
\end{abstract}

*Laboratoire de Mathématiques, UMR CNRS 8628, Université Paris-Sud, Bâtiment 425, F-91405 Orsay, France.

${ }^{\dagger}$ IRMAR, UMR CNRS 6625, Université de Rennes 1, Campus de Beaulieu, F-35042 Rennes Cedex, France. 


\section{Introduction}

We are interested in the exponentially small eigenvalues of the Dirichlet realization of the semiclassical Witten Laplacian on 0-forms

$$
\Delta_{f, h}^{(0)}=-h^{2} \Delta+|\nabla f(x)|^{2}-h \Delta f(x) .
$$

Our aim is to extend to the case of a regular bounded open set $\Omega$, or more generally a compact manifold with boundary, results which were previously obtained in the case when $\Omega$ is a compact manifold or the case of $\mathbb{R}^{n}$. We shall analyze the Dirichlet realization of this operator.

The function $f$ is assumed to be a Morse function on $\bar{\Omega}$ (with no critical points at the boundary). It is known (see [Sim2], [Wit], [CFKS], [HelSj4] and more recently [CL]) that, like in the case without boundary, there are exactly $m_{0}$ eigenvalues in some interval $\left[0, C h^{\frac{6}{5}}\right]$ for $h>0$ small enough, where $m_{0}$ is the number of local minima in $\Omega$. This is strongly due to the fact that the Dirichlet case is concerned. These eigenvalues are actually exponentially small as $h \rightarrow 0$.

Moreover this can be extended (see [CL]) to Laplacians on $p$-forms, $p>1$, but this time some critical points of the restriction of the Morse function to the boundary (which will be assumed to be a Morse function) will play a role.

Our purpose is to derive with the same accuracy as in [HKN] asymptotic formulas for the $m_{0}$ first eigenvalues of the Dirichlet realization of $\Delta_{f, h}^{(0)}$. A similar problem was considered by many authors via a probabilistic approach in [FrWe], [HolKusStr], [Mic], [Kol]. More recently, in the case of $\mathbb{R}^{n}$, A. Bovier, M. Eckhoff, V. Gayrard and M. Klein obtained in [BEGK] and [BoGayKl], accurate asymptotic forms of the exponentially small eigenvalues. These results were improved and extended to the case of a compact manifold in [HKN].

The Witten Laplacian is associated to the Dirichlet form

$$
C_{0}^{\infty}(\Omega) \ni u \mapsto \int_{\Omega}|(h \nabla+\nabla f) u(x)|^{2} d x
$$

Note that the probabilists look equivalently at:

$$
C_{0}^{\infty}(\Omega) \ni v \mapsto h^{2} \int_{\Omega}|\nabla v(x)|^{2} e^{-2 f(x) / h} d x
$$


Bovier, Eckhoff, Gayrard and Klein considered this problem via a probabilistic approach. They obtained, in the case of $\mathbb{R}^{n}$ and under additional conditions on $f$ and $\nabla f$ at $\infty$, the following asymptotic behavior for the first eigenvalues $\lambda_{k}(h), k \in\left\{2, \ldots, m_{0}\right\}$, with $\lambda_{1}(h)=0$, of $\Delta_{f, h}^{(0)}$ :

$$
\begin{aligned}
\lambda_{k}(h)=\frac{h}{\pi}\left|\widehat{\lambda}_{1}\left(U_{j(k)}^{(1)}\right)\right| \sqrt{\frac{\left|\operatorname{det}\left(\operatorname{Hess} f\left(U_{k}^{(0)}\right)\right)\right|}{\left|\operatorname{det}\left(\operatorname{Hess} f\left(U_{j(k)}^{(1)}\right)\right)\right|}} \\
\quad \times \exp -\frac{2}{h}\left(f\left(U_{j(k)}^{(1)}\right)-f\left(U_{k}^{(0)}\right)\right) \times\left(1+\mathcal{O}\left(h^{\frac{1}{2}}|\log h|\right)\right),
\end{aligned}
$$

where the $U_{k}^{(0)}$ denote the local minima of $f$ ordered in some specific way, the $U_{j(k)}^{(1)}$ are "saddle points" attached in a specific way to the $U_{k}^{(0)}$ (which appear to be critical points of index 1) and $\widehat{\lambda}_{1}\left(U_{j(k)}^{(1)}\right)$ is the negative eigenvalue of Hess $f\left(U_{j(k)}^{(1)}\right)$.

Their article belongs to a family of works done by probabilists starting at least from Freidlin and Wentzel (See [FrWe] for a presentation). The first papers were only giving the asymptotic behavior of the logarithm of the eigenvalues. The main contribution of [BoGayKl] and [BEGK] was to determine the main term in the prefactor. The later [HKN] gave a complete asymptotics in (1.1) and extended the results to more general geometries, including cases when $\lambda_{1}(h) \neq 0$.

In the case with boundary, we observe that the function $\exp -\frac{f}{h}$ does not satisfy the Dirichlet condition, so the smallest eigenvalue can not be 0. For this case, we can mention as starting reference Theorem 7.4 in [FrWe], which says (in particular) that, if $f$ has a unique non degenerate local minimum $x_{\text {min }}$, then the lowest eigenvalue $\lambda_{1}(h)$ of the Dirichlet realization $\Delta_{f, h}^{(0)}$ in $\Omega$ satisfies :

$$
\lim _{h \rightarrow 0}-h \log \lambda_{1}(h)=\inf _{x \in \partial \Omega}\left(f(x)-f\left(x_{\text {min }}\right)\right) .
$$

Other results are given in the case of many local minima but they are limited to the determination of logarithmic equivalents (see Theorems 7.3 and 7.4 in $[\mathrm{FrWe}])$.

The approach given in [HKN] intensively uses, together with the techniques of [HelSj4], the two facts that the Witten Laplacian is associated to 
a cohomology complex and that the function $x \mapsto \exp -\frac{f(x)}{h}$ is a distribution solution in the kernel of the Witten Laplacian on 0 -forms permitting to construct very efficiently quasimodes. We recall that the Witten Laplacian is defined as

$$
\Delta_{f, h}=d_{f, h} d_{f, h}^{*}+d_{f, h}^{*} d_{f, h},
$$

where $d_{f, h}$ is the distorted differential

$$
d_{f, h}:=e^{-f(x) / h}\left(h d_{x}\right) e^{f(x) / h},
$$

and where $d_{f, h}^{*}$ is its adjoint for the $L^{2}$-scalar product canonically associated to the Riemannian structure. The restriction of $d_{f, h}$ to $p$-forms is denoted by $d_{f, h}^{(p)}$. With these notations, the Witten Laplacian on functions is

$$
\Delta_{f, h}^{(0)}=d_{f, h}^{(0) *} d_{f, h}^{(0)} .
$$

In the Witten-complex spirit and due to the relation

$$
d_{f, h}^{(0)} \Delta_{f, h}^{(0)}=\Delta_{f, h}^{(1)} d_{f, h}^{(1)},
$$

it is more convenient to consider the singular values of the restricted differential $d_{f, h}^{(0)}: F^{(0)} \rightarrow F^{(1)}$. The space $F^{(\ell)}$ is the $m_{\ell^{-}}$dimensional spectral subspace of $\Delta_{f, h}^{(\ell)}, \ell \in\{0,1\}$,

$$
F^{(\ell)}=\operatorname{Ran} 1_{I(h)}\left(\Delta_{f, h}^{(\ell)}\right)
$$

with $I(h)=\left[0, C h^{\frac{6}{5}}\right]$ and the property ${ }^{1}$

$$
1_{I(h)}\left(\Delta_{f, h}^{(1)}\right) d_{f, h}^{(0)}=d_{f, h} 1_{I(h)}^{(0)}\left(\Delta_{f, h}^{(0)}\right) .
$$

The restriction $\left.d_{f, h}\right|_{F^{(\ell)}}$ will be more shortly denoted by $\beta_{f, h}^{(\ell)}$

$$
\beta_{f, h}^{(\ell)}:=\left(d_{f, h}^{(\ell)}\right)_{/ F^{(\ell)}} .
$$

We will mainly concentrate on the case $\ell=0$.

In order to exploit all the information which can be extracted from well chosen quasimodes, working with singular values of $\beta_{f, h}^{(0)}$ happens to be more efficient than considering their squares, the eigenvalues of $\Delta_{f, h}^{(0)}$. Those quantities agree better with the underlying Witten complex structure.

\footnotetext{
${ }^{1}$ The right end $a(h)=C h^{\frac{6}{5}}$ of the interval $I(h)=[0, a(h)]$ is suitable for technical reasons. What is important is that $a(h)=o(h)$. The value of $C>0$ does not play any role.
} 


\section{The main result}

Let us describe the result. We shall show that under a suitable generic assumption (see Assumption 5.7), one can label the $m_{0}$ local minima and introduce an injective map $j$ from the set of the local minima into the set of the $m_{1}$ generalized critical points with index 1 of the Morse functions in $\bar{\Omega}$. At a generalized critical point $U$ with index 1 , we can associate the Hessians Hess $f(U)$, if $U \in \Omega$, or $\left(\left.\operatorname{Hess} f\right|_{\partial \Omega}\right)(U)$, if $U \in \partial \Omega$. When $U \in \Omega, \widehat{\lambda}_{1}(U)$ denotes the negative eigenvalue of $\operatorname{Hess} f(U)$.

\section{Theorem 1.1.}

Under Assumption (5.7), there exists $h_{0}$ such that, for $h \in\left(0, h_{0}\right]$, the spectrum in $\left[0, h^{\frac{3}{2}}\right)$ of the Dirichlet realization of $\Delta_{f, h}^{(0)}$ in $\Omega$, consists of $m_{0}$ eigenvalues $\lambda_{1}(h)<\ldots<\lambda_{m_{0}}(h)$ of multiplicity 1 , which are exponentially small and admit the following asymptotic expansions :

$$
\begin{aligned}
\lambda_{k}(h)=\frac{h}{\pi}\left|\widehat{\lambda}_{1}\left(U_{j(k)}^{(1)}\right)\right| \sqrt{\frac{\left|\operatorname{det}\left(\operatorname{Hess} f\left(U_{k}^{(0)}\right)\right)\right|}{\mid \operatorname{det}\left(\operatorname{Hess} f\left(U_{j(k)}^{(1)}\right) \mid\right.}}\left(1+h c_{k}^{1}(h)\right) \times \\
\quad \times \exp -\frac{2}{h}\left(f\left(U_{j(k)}^{(1)}\right)-f\left(U_{k}^{(0)}\right)\right), \quad \text { if } U_{j(k)}^{(1)} \in \Omega,
\end{aligned}
$$

and

$$
\begin{aligned}
\lambda_{k}(h)=\frac{2 h^{1 / 2}\left|\nabla f\left(U_{j(k)}^{(1)}\right)\right|}{\pi^{1 / 2}} & \sqrt{\frac{\left|\operatorname{det}\left(\operatorname{Hess} f\left(U_{k}^{(0)}\right)\right)\right|}{\left|\operatorname{det}\left(\left.\operatorname{Hess} f\right|_{\partial \Omega}\left(U_{j(k)}^{(1)}\right)\right)\right|}}\left(1+h c_{k}^{1}(h)\right) \times \\
\times & \exp -\frac{2}{h}\left(f\left(U_{j(k)}^{(1)}\right)-f\left(U_{k}^{(0)}\right)\right), \quad \text { if } U_{j(k)}^{(1)} \in \partial \Omega,
\end{aligned}
$$

where $c_{k}^{1}(h)$ admits a complete expansion : $c_{k}^{1}(h) \sim \sum_{m=0}^{\infty} h^{m} c_{k, m}$.

This theorem extends to the case with boundary the previous result of [BoGayKl] and its improvement in [HKN] (see also non rigorous formal computations of [KolMa], who look also to cases with symmetry and the books $[\mathrm{FrWe}]$ and $[\mathrm{Kol}]$ and references therein).

\section{About the proof}

As in $[\mathrm{HelSj} 4]$ and $[\mathrm{HKN}]$, the proof will be deeply connected with the analysis 
of the small eigenvalues of a suitable realization (which is not the Dirichlet realization) of the Laplacian on the 1 -forms. In order to follow the same strategy as in the boundaryless case, three main points have to be explained. The first point was to find the right substitute for the Witten complex. Our starting problem being the analysis of the Dirichlet realization of the Witten Laplacian, we were led to find the right realization of the Witten Laplacian on 1-forms in the case with boundary in order to keep the commutation relation (1.6). The answer was present in the literature ([Schw], $[\mathrm{Gu}]$ and [CL]) in connection with the analysis of the relative cohomology.

The second point was to get the "rough" localization of the spectrum of this Laplacian on 1-forms. The analysis was performed in [CL], in the spirit of Witten's idea, extending the so called harmonic approximation. But these authors, interested in the Morse theory, simplified the problem in the sense that they use the possibility (inherent to Morse theory) to choose a right metric and a right Morse function in order to simplify the analysis at the boundary. We emphasize that we treat here the general case.

The third point is the construction of WKB solutions for the critical points of the restriction of the Morse function at the boundary. For simplicity, we restrict our attention to the case of 1 -forms which is the only one needed for our problem.

\section{Structure of the paper}

The paper is organized as follows. In the second section, we analyze in detail the boundary complex adapted to our analysis. The third section is devoted to the proof of rough estimates replacing the harmonic oscillator approximation in the case without boundary (leading in particular to the proof of the weak Morse Inequalities). In the fourth section, we give the WKB construction for a solution of the Witten Laplacian on 1-forms localized at a critical point of the boundary. The fifth section is devoted to the Morse theory together with the right definition of saddle sets in the present case with boundary. This permits us in particular to explain our main assumptions. The sixth section is devoted to the construction of quasimodes and the proof of the main theorem is given in the seventh section. Finally, we have given in the appendix a partially independent treatment of the one-dimensional case, which can be seen as a warm-up. 


\section{Some self-adjoint realization of Witten Lapla- cians with boundary.}

\section{$2.1 \quad$ Introduction}

We work here on a $\mathcal{C}^{\infty}$ connected compact oriented Riemannian manifold $\bar{\Omega}$ with boundary $\partial \Omega$ and $\Omega$ will denote its interior. After fixing basic notations we specify the self-adjoint realization of the Witten Laplacian on which we will focus and we assume in all the paper that the function $f$ is a $\mathcal{C}^{\infty}$ real valued function on $\bar{\Omega}$.

\subsection{Distorted differentials and associated Witten Lapla- cians.}

The cotangent (resp. tangent) bundle on $\Omega$ is denoted by $T^{*} \Omega$ (resp. $T \Omega$ ) and the exterior fiber bundle by $\Lambda T^{*} \Omega=\oplus_{p=0}^{n} \Lambda^{p} T^{*} \Omega\left(\operatorname{resp} . \Lambda T \Omega=\oplus_{p=0}^{n} \Lambda^{p} T \Omega\right)$. The fiber bundles $\Lambda T \partial \Omega=\oplus_{p=0}^{n-1} \Lambda^{p} T \partial \Omega$ and $\Lambda T^{*} \partial \Omega=\oplus_{p=0}^{n-1} \Lambda^{p} T^{*} \partial \Omega$ are defined similarly. The space of $\mathcal{C}^{\infty}, \mathcal{C}_{0}^{\infty}, L^{2}, H^{s} \ldots$ sections in any of these fiber bundles, $E$, on $O=\Omega$ or $O=\partial \Omega$, will be denoted respectively by $\mathcal{C}^{\infty}(O ; E), \mathcal{C}_{0}^{\infty}(O ; E), L^{2}(O ; E), H^{s}(O ; E) \ldots$ When no confusion is possible we will simply use the short notations $\Lambda^{p} \mathcal{C}^{\infty}, \Lambda^{p} \mathcal{C}_{0}^{\infty}, \Lambda^{p} L^{2}$ and $\Lambda^{p} H^{s}$ for $E=\Lambda^{p} T^{*} \Omega$ or $E=\Lambda^{p} T^{*} \partial \Omega$. Note that the $L^{2}$ spaces are those associated with the unit volume form for the Riemannian structure on $\Omega$ or $\partial \Omega(\Omega$ and $\partial \Omega$ are oriented $)$. The notation $\mathcal{C}^{\infty}(\bar{\Omega} ; E)$ is used for the set of $\mathcal{C}^{\infty}$ sections up to the boundary. Finally since $\partial \Omega$ is $\mathcal{C}^{\infty}, \mathcal{C}^{\infty}(\bar{\Omega} ; E)$ is dense in $H^{s}(\Omega ; E)$ for $s \geq 0$ and the trace operator $\left.\omega \rightarrow \omega\right|_{\partial \Omega}$ extends to a surjective operator from $H^{s}(\Omega ; E)$ onto $H^{s-1 / 2}(\partial \Omega ; E)$ as soon as $s>1 / 2$.

The differential on $\mathcal{C}_{0}^{\infty}\left(\Omega ; \Lambda T^{*} \Omega\right)$ will be denoted by $d$ and more precisely

$$
d^{(p)}: \mathcal{C}_{0}^{\infty}\left(\Omega ; \Lambda^{p} T^{*} \Omega\right) \rightarrow \mathcal{C}_{0}^{\infty}\left(\Omega ; \Lambda^{p+1} T^{*} \Omega\right) .
$$

Its formal adjoint with respect the $L^{2}$-scalar product inherited from the Riemannian structure is denoted by $d^{*}$ with

$$
d^{(p), *}: \mathcal{C}_{0}^{\infty}\left(\Omega ; \Lambda^{p+1} T^{*} \Omega\right) \rightarrow \mathcal{C}_{0}^{\infty}\left(\Omega ; \Lambda^{p} T^{*} \Omega\right)
$$

Those differential $d$ and codifferential $d^{*}$ are well defined on $\mathcal{C}^{\infty}\left(\bar{\Omega} ; \Lambda T^{*} \Omega\right)$ and satisfy $d d=d^{*} d^{*}=0$. 
For a function $f \in \mathcal{C}^{\infty}(\bar{\Omega} ; \mathbb{R})$ and $h>0$, we set

$$
d_{f, h}=e^{-f(x) / h}(h d) e^{f(x) / h} \quad \text { and } \quad d_{f, h}^{*}=e^{f(x) / h}\left(h d^{*}\right) e^{-f(x) / h} .
$$

The Witten Laplacian is the differential operator defined on $\mathcal{C}^{\infty}\left(\bar{\Omega} ; \Lambda T^{*} \Omega\right)$

$$
\Delta_{f, h}=d_{f, h}^{*} d_{f, h}+d_{f, h} d_{f, h}^{*}=\left(d_{f, h}+d_{f, h}^{*}\right)^{2}
$$

which means, by restriction to the $p$-forms in $\mathcal{C}^{\infty}\left(\bar{\Omega} ; \Lambda^{p} T^{*} \Omega\right)$,

$$
\Delta_{f, h}^{(p)}=d_{f, h}^{(p), *} d_{f, h}^{(p)}+d_{f, h}^{(p-1)} d_{f, h}^{(p-1), *}
$$

Note that $d_{f, h} d_{f, h}=0$, and $d_{f, h}^{*} d_{f, h}^{*}=0$ respectively, imply that, for all $u$ in $\mathcal{C}^{\infty}\left(\bar{\Omega} ; \Lambda^{p} T^{*} \Omega\right)$,

$$
\begin{gathered}
\Delta_{f, h}^{(p+1)} d_{f, h}^{(p)} u=d_{f, h}^{(p)} \Delta_{f, h}^{(p)} u \\
\text { and } \\
\Delta_{f, h}^{(p-1)} d_{f, h}^{(p-1), *} u=d_{f, h}^{(p-1), *} \Delta_{f, h}^{(p)} u .
\end{gathered}
$$

Here are other relations with exterior and interior products and Lie derivatives which will be useful :

$$
\begin{gathered}
d_{f, h}=h d+d f \wedge ; \\
d_{f, h}^{*}=h d^{*}+\mathbf{i}_{\nabla f} ; \\
d \circ \mathbf{i}_{X}+\mathbf{i}_{X} d=\mathcal{L}_{X} ; \\
\Delta_{f, h}=h^{2}\left(d+d^{*}\right)^{2}+|\nabla f|^{2}+h\left(\mathcal{L}_{\nabla f}+\mathcal{L}_{\nabla f}^{*}\right) .
\end{gathered}
$$

\subsection{Stokes formulas.}

Before writing the distorted Stokes formula, we recall some notations which are convenient for boundary problems even with the euclidean metric on $\Omega \subset \mathbb{R}^{n}$. We refer the reader to [Schw] for details.

For any $\omega \in \mathcal{C}^{\infty}\left(\bar{\Omega} ; \Lambda^{p} T^{*} \Omega\right)$, the form $\mathbf{t} \omega$ is the element of $\mathcal{C}^{\infty}\left(\partial \Omega ; \Lambda^{p} T^{*} \Omega\right)$ defined by

$$
(\mathbf{t} \omega)_{\sigma}\left(X_{1}, \ldots, X_{p}\right)=\omega_{\sigma}\left(X_{1}^{T}, \ldots, X_{p}^{T}\right), \forall \sigma \in \partial \Omega
$$


with the decomposition into the tangential and normal components to $\partial \Omega$ at $\sigma: X_{i}=X_{i}^{T} \oplus X_{i}^{\perp} n_{\sigma}$.

If $n_{\sigma}^{*}$ denotes the 1-form which is dual to the outgoing normal $n_{\sigma}$ at $\sigma$ for the Riemannian scalar product, we have

$$
(\mathbf{t} \omega)_{\sigma}=\mathbf{i}_{n_{\sigma}}\left(n_{\sigma}^{*} \wedge \omega_{\sigma}\right) .
$$

Note that $\mathbf{t} \omega$ can be identified with $j^{*} \omega \in \mathcal{C}^{\infty}\left(\partial \Omega ; \Lambda^{p} T^{*} \partial \Omega\right)$ where $j: \partial \Omega \rightarrow \Omega$ is the canonical injection.

The non tangential part of $\omega$ on $\partial \Omega$ is defined by

$$
\mathbf{n} \omega=\left.\omega\right|_{\partial \Omega}-\mathbf{t} \omega \quad \in \mathcal{C}^{\infty}\left(\partial \Omega ; \Lambda^{p} T^{*} \Omega\right) .
$$

If necessary $\mathbf{t} \omega$ and $\mathbf{n} \omega$ can be considered as elements of $\mathcal{C}^{\infty}\left(\bar{\Omega} ; \Lambda^{p} T^{*} \Omega\right)$ as follows. A variant of the Collar Theorem which provides a diffeomorphism between a neighborhood of $\partial \Omega$ and $\partial \Omega \times[0, \delta[, \delta>0$ small enough, can be written by taking for the normal coordinate the geodesic distance to $\partial \Omega$, $x_{n}=d_{\Omega}(x, \partial \Omega) \in\left[0, \delta\left[\right.\right.$. Any form $\eta \in \mathcal{C}^{\infty}\left(\partial \Omega ; \Lambda T^{*} \Omega\right)$ is then extended by using the equation $\partial_{x_{n}} \eta=0$ to $\partial \Omega \times[0, \delta[$. After multiplication by a cut-off function, this gives a form on $\Omega$, which does not depend on $x_{n}$ in a neighborhood of $\partial \Omega$.

The Hodge operator $\star$ is locally defined in a local orthonormal frame $\left(E_{1}, \ldots, E_{n}\right)$ by

$$
\left(\star \omega_{x}\right)\left(E_{\sigma(p+1)}, \ldots, E_{\sigma(n)}\right)=\varepsilon(\sigma) \omega_{x}\left(E_{\sigma(1)}, \ldots, E_{\sigma(p)}\right),
$$

for $\omega_{x} \in \Lambda^{p} T_{x}^{*} \Omega$ and with $\sigma \in \Sigma(n)$ preserving $\{1, \ldots, p\}$.

We recall the formulas

$$
\begin{gathered}
\star\left(\star \omega_{x}\right)=(-1)^{p(n-p)} \omega_{x}, \quad \forall \omega_{x} \in \Lambda^{p} T_{x}^{*} \Omega, \\
\left\langle\omega_{1} \mid \omega_{2}\right\rangle_{\Lambda^{p} L^{2}}=\int_{\Omega} \omega_{1} \wedge \star \overline{\omega_{2}}, \quad \forall \omega_{1}, \omega_{1} \in \Lambda^{p} L^{2},
\end{gathered}
$$

and

$$
\begin{aligned}
\star d^{*,(p-1)}=(-1)^{p} d^{(n-p)} \star, & \star d^{(p)}=(-1)^{p+1} d^{*,(n-p-1)} \star, \\
\star \mathbf{n}=\mathbf{t} \star, & \star \mathbf{t}=\mathbf{n} \star, \\
\mathbf{t} d=d \mathbf{t}, & \mathbf{n} d^{*}=d^{*} \mathbf{n} .
\end{aligned}
$$

These formulas, combined with the Stokes formula,

$$
\forall \omega \in \mathcal{C}^{\infty}\left(\bar{\Omega} ; \Lambda^{p} T^{*} \Omega\right), \quad \int_{\Omega} d \omega=\int_{\partial \Omega} j^{*} \omega=\int_{\partial \Omega} \mathbf{t} \omega,
$$

lead to the Green formula. 


\section{Lemma 2.1.}

For all $\omega \in \Lambda^{p} H^{2}$ and $\eta \in \Lambda^{p} H^{1}$, we have

$$
\begin{aligned}
& \left\langle d_{f, h} \omega \mid d_{f, h} \eta\right\rangle_{\Lambda^{p+1} L^{2}}+\left\langle d_{f, h}^{*} \omega \mid d_{f, h}^{*} \eta\right\rangle_{\Lambda^{p-1} L^{2}} \\
& =\left\langle\Delta_{f, h} \omega \mid \eta\right\rangle_{\Lambda^{p} L^{2}}+h \int_{\partial \Omega}(\mathbf{t} \bar{\eta}) \wedge\left(\star \mathbf{n} d_{f, h} \omega\right)-h \int_{\partial \Omega}\left(\mathbf{t} d_{f, h}^{*} \omega\right) \wedge(\star \mathbf{n} \bar{\eta}) .
\end{aligned}
$$

Proof.

Since $\mathcal{C}^{\infty}\left(\bar{\Omega} ; \Lambda T^{*} \Omega\right)$ is dense in $\Lambda H^{s}$, while both terms of the identity are bilinearly continuous on $\Lambda^{p} H^{2} \times \Lambda^{p} H^{1}$, the forms $\omega$ and $\eta$ can be assumed $\mathcal{C}^{\infty}$ up to the boundary.

We write

$$
\begin{aligned}
& \left\langle d_{f, h} \omega \mid d_{f, h} \eta\right\rangle+\left\langle d_{f, h}^{*} \omega \mid d_{f, h}^{*} \eta\right\rangle=\langle h d \omega \mid h d \eta\rangle+\left\langle h d^{*} \omega \mid h d^{*} \eta\right\rangle \\
& +\langle d f \wedge \omega \mid h d \eta\rangle+\langle h d \omega \mid d f \wedge \eta\rangle+\langle d f \wedge \omega \mid d f \wedge \eta\rangle \\
& +\left\langle\mathbf{i}_{\nabla f} \omega \mid h d^{*} \eta\right\rangle+\left\langle h d^{*} \omega \mid \mathbf{i}_{\nabla f} \eta\right\rangle+\left\langle\mathbf{i}_{\nabla f} \omega \mid \mathbf{i}_{\nabla f} \eta\right\rangle .
\end{aligned}
$$

Let us first compute

$$
\begin{aligned}
\langle d f \wedge \omega \mid d f \wedge \eta\rangle+\left\langle\mathbf{i}_{\nabla f} \omega \mid \mathbf{i}_{\nabla f} \eta\right\rangle=\left\langle\mathbf{i}_{\nabla f}(d f\right. & \wedge \omega)+d f \wedge\left(\mathbf{i}_{\nabla f} \omega\right)|\eta\rangle \\
& =\left\langle\left(\mathbf{i}_{\nabla f} d f\right) \omega \mid \eta\right\rangle=\left\langle|\nabla f|^{2} \omega \mid \eta\right\rangle
\end{aligned}
$$

according to the identity

$$
\mathbf{i}_{X}(\alpha \wedge \beta)=\left(\mathbf{i}_{X} \alpha\right) \wedge \beta+(-1)^{\operatorname{deg} \alpha} \alpha \wedge\left(\mathbf{i}_{X} \beta\right) .
$$

The Stokes formula, combined with

$$
\left\langle\theta_{1} \mid d^{*} \theta_{2}\right\rangle_{x} d x_{1} \wedge \ldots \wedge d x_{n}=\theta_{1} \wedge \star d^{*} \overline{\theta_{2}}=\theta_{1} \wedge(-1)^{\operatorname{deg} \theta_{2}} d\left(\star \overline{\theta_{2}}\right)
$$

and

$$
d\left(\theta_{2} \wedge \star \overline{\theta_{2}}\right)=d \theta_{1} \wedge\left(\star \overline{\theta_{2}}\right)+(-1)^{\operatorname{deg} \theta_{1}} \theta_{1} \wedge d\left(\star \overline{\theta_{2}}\right)
$$

where $\left(d x_{1}, \ldots, d x_{n}\right)$ is orthonormal with a positive orientation, yields for $\operatorname{deg} \theta_{1}=\operatorname{deg} \theta_{2} \mp 1$ :

$$
\int_{\partial \Omega} \mathbf{t}\left[\theta_{1} \wedge\left(\star \overline{\theta_{2}}\right)\right]=\left\langle d \theta_{1} \mid \theta_{2}\right\rangle-\left\langle\theta_{1} \mid d^{*} \theta_{2}\right\rangle
$$

and

$$
\int_{\partial \Omega} \mathbf{t}\left[\overline{\theta_{2}} \wedge\left(\star \theta_{1}\right)\right]=\left\langle\theta_{1} \mid d \theta_{2}\right\rangle-\left\langle d^{*} \theta_{1} \mid \theta_{2}\right\rangle
$$


From the first identity we deduce :

$$
\begin{aligned}
\left\langle h d^{*} \omega \mid h d^{*} \eta\right\rangle+\left\langle\mathbf{i}_{\nabla f} \omega\right| & \left.h d^{*} \eta\right\rangle=\left\langle h^{2} d d^{*} \omega \mid \eta\right\rangle+\left\langle h d \mathbf{i}_{\nabla f} \omega \mid \eta\right\rangle \\
& -h \int_{\partial \Omega} \mathbf{t}\left[\left(h d^{*} \omega+\mathbf{i}_{\nabla f} \omega\right) \wedge \star \bar{\eta}\right] \\
= & \left\langle h^{2} d d^{*} \omega \mid \eta\right\rangle+\left\langle h d \mathbf{i}_{\nabla f} \omega \mid \eta\right\rangle-h \int_{\partial \Omega}\left(\mathbf{t} d_{f, h}^{*} \omega\right) \wedge(\star \mathbf{n} \bar{\eta}) .
\end{aligned}
$$

From the second one we get :

$$
\begin{gathered}
\langle h d \omega \mid h d \eta\rangle+\langle d f \wedge \omega \mid h d \eta\rangle=\left\langle h^{2} d^{*} d \omega \mid \eta\right\rangle+\left\langle h d^{*}(d f \wedge \omega) \mid \eta\right\rangle \\
+h \int_{\partial \Omega} \mathbf{t}[\bar{\eta} \wedge \star(h d \omega+d f \wedge \omega)] \\
=\left\langle h^{2} d^{*} d \omega \mid \eta\right\rangle+\left\langle h d^{*}(d f \wedge \omega) \mid \eta\right\rangle+h \int_{\partial \Omega}(\mathbf{t} \bar{\eta}) \wedge\left(\star \mathbf{n} d_{f, h} \omega\right) .
\end{gathered}
$$

Finally the relations (cf $(2.3),(2.4))$

$$
\mathbf{i}_{\nabla f} \circ d+d \circ \mathbf{i}_{\nabla f}=\mathcal{L}_{\nabla f} \quad \text { and } \quad d^{*} \circ(d f \wedge)+(d f \wedge) \circ d^{*}=\mathcal{L}_{\nabla f}^{*}
$$

lead to

$$
\begin{aligned}
\left\langle d_{f, h} \omega \mid d_{f, h} \eta\right\rangle_{\Lambda^{p+1} L^{2}} & \left\langle\left\langle d_{f, h}^{*} \omega \mid d_{f, h}^{*} \eta\right\rangle_{\Lambda^{p-1} L^{2}}\right. \\
=\left\langle h^{2}(d+\right. & \left.d^{*}\right)^{2}+|\nabla f|^{2}+h\left(\mathcal{L}_{\nabla f}+\mathcal{L}_{\nabla f}^{*}\right) \omega|\eta\rangle_{\Lambda^{p} L^{2}} \\
& \quad+h \int_{\partial \Omega}(\mathbf{t} \bar{\eta}) \wedge\left(\star \mathbf{n} d_{f, h} \omega\right)-h \int_{\partial \Omega}\left(\mathbf{t} d_{f, h}^{*} \omega\right) \wedge(\star \mathbf{n} \bar{\eta}),
\end{aligned}
$$

where the differential operator $h^{2}\left(d+d^{*}\right)^{2}+|\nabla f|^{2}+h\left(\mathcal{L}_{\nabla f}+\mathcal{L}_{\nabla f}^{*}\right)$ is nothing but $\Delta_{f, h}$.

Note that this writing does not depend on the choice of an orientation. If $\mu$ and $\mu_{\partial \Omega}$ denote the volume form in $\Omega$ and $\partial \Omega$ and if the normal vector $n_{\sigma}$ is chosen according to $\left(\mu_{\partial \Omega}\right)_{\sigma}\left(X_{1}, \ldots, X_{n-1}\right)=\mu_{\sigma}\left(n_{\sigma}, X_{1}, \ldots, X_{n-1}\right)$, a simple computation in normal frames leads to

$$
\mathbf{t} \omega_{1} \wedge \star \mathbf{n} \overline{\omega_{2}}=\left\langle\omega_{1} \mid \mathbf{i}_{n_{\sigma}} \omega_{2}\right\rangle_{\Lambda^{p} T_{\sigma}^{*} \Omega} d \mu_{\partial \Omega},
$$


for $\omega_{1} \in \mathcal{C}^{\infty}\left(\bar{\Omega} ; \Lambda^{p} T^{*} \Omega\right)$ and $\omega_{2} \in C^{\infty}\left(\bar{\Omega} ; \Lambda^{p+1} T^{*} \Omega\right)$.

After choosing for $n_{\sigma}$ the outgoing normal vector, (2.12) is equivalent to

$$
\begin{aligned}
& \left\langle\Delta_{f, h} \omega, \eta\right\rangle_{\Lambda^{p} L^{2}}=\left\langle d_{f, h} \omega \mid d_{f, h} \eta\right\rangle_{\Lambda^{p+1} L^{2}}+\left\langle d_{f, h}^{*} \omega \mid d_{f, h}^{*} \eta\right\rangle_{\Lambda^{p-1} L^{2}} \\
& -h \int_{\partial \Omega}\left\langle\mathbf{i}_{n_{\sigma}} d_{f, h} \omega \mid \eta\right\rangle_{\Lambda^{p} T_{\sigma}^{*} \Omega}(\sigma) d \mu_{\partial \Omega}+h \int_{\partial \Omega}\left\langle n_{\sigma}^{*} \wedge d_{f, h}^{*} \omega \mid \eta\right\rangle_{\Lambda^{p} T_{\sigma}^{*} \Omega}(\sigma) d \mu_{\partial \Omega},
\end{aligned}
$$

which was used in [HelSj4] (see Lemma 1.1, p. 255, with the inward normal vector).

As a consequence of (2.13) we get the following useful decomposition formula.

\section{Lemma 2.2.}

If $n_{\sigma}$ denotes the exterior normal vector at $\sigma \in \partial \Omega$, and $(\partial f / \partial n)(\sigma)=$ $n_{\sigma} \cdot \nabla f(\sigma)$ is the normal derivative of $f$ at $\sigma$, then the identity

$$
\begin{gathered}
\left\|d_{f, h} \omega\right\|_{\Lambda^{p+1} L^{2}}^{2}+\left\|d_{f, h}^{*} \omega\right\|_{\Lambda^{p-1} L^{2}}^{2}=h^{2}\|d \omega\|_{\Lambda^{p+1} L^{2}}^{2}+h^{2}\left\|d^{*} \omega\right\|_{\Lambda^{p-1} L^{2}}^{2}+\||\nabla f| \omega\|_{\Lambda^{p} L^{2}}^{2} \\
+h\left\langle\left(\mathcal{L}_{\nabla f}+\mathcal{L}_{\nabla f}^{*}\right) \omega \mid \omega\right\rangle_{\Lambda^{p} L^{2}}-h \int_{\partial \Omega}\langle\omega \mid \omega\rangle_{\Lambda^{p} T_{\sigma}^{*} \Omega}\left(\frac{\partial f}{\partial n}\right)(\sigma) d \mu_{\partial \Omega} \quad(2.15)
\end{gathered}
$$

holds for any $\omega \in \Lambda^{p} H^{1}$ such that $\mathbf{t} \omega=0$.

Proof.

Again both sides of the identity are continuous on $\Lambda^{p} H^{1}$ and we can assume $\omega \in \mathcal{C}^{\infty}\left(\bar{\Omega} ; \Lambda^{p} T^{*} \Omega\right)$.

We use the relation (2.12) with $f$ replaced by $0, d_{0, h}=h d$ and $d_{0, h}^{*}=h d^{*}$.

We obtain

$$
\begin{aligned}
& \left\|d_{f, h} \omega\right\|_{\Lambda^{p+1} L^{2}}^{2}+\left\|d_{f, h}^{*} \omega\right\|_{\Lambda^{p-1} L^{2}}^{2}-h^{2}\|d \omega\|_{\Lambda^{p+1} L^{2}}^{2}-h^{2}\left\|d_{f, h}^{*} \omega\right\|_{\Lambda^{p-1} L^{2}}^{2}= \\
& \left\langle\left(\Delta_{f, h}-\Delta_{0, h}\right) \omega \mid \omega\right\rangle_{\Lambda^{p} L^{2}}+h \int_{\partial \Omega}(\mathbf{t} \bar{\omega}) \wedge \star \mathbf{n}(d f \wedge \omega)-h \int_{\partial \Omega}\left(\mathbf{t i}_{\nabla f} \omega\right) \wedge(\star \mathbf{n} \bar{\omega}) \\
& =\left\langle\left(\Delta_{f, h}-\Delta_{0, h}\right) \omega \mid \omega\right\rangle_{\Lambda^{p} L^{2}}-h \int_{\partial \Omega}\left\langle\mathbf{i}_{\nabla f} \omega \mid \mathbf{i}_{n_{\sigma}} \omega\right\rangle_{\Lambda T_{\sigma}^{*} \Omega} d \mu_{\partial \Omega} .
\end{aligned}
$$

The first term of the right-hand side equals

$$
\left\langle\left(\Delta_{f, h}-\Delta_{0, h}\right) \omega \mid \omega\right\rangle_{\Lambda^{p} L^{2}}=\||\nabla f| \omega\|_{\Lambda^{p} L^{2}}^{2}+h\left\langle\left(\mathcal{L}_{\nabla f}+\mathcal{L}_{\nabla f}^{*}\right) \omega \mid \omega\right\rangle_{\Lambda^{p} L^{2}} .
$$

For the integral term, we write

$$
\mathbf{i}_{\nabla f} \omega=\frac{\partial f}{\partial n}(\sigma) \mathbf{i}_{n_{\sigma}} \omega+\mathbf{i}_{\nabla_{T} f} \omega
$$


where $\nabla_{T} f$ denotes the tangential part of the gradient. The equality

$$
\mathbf{t i}_{\nabla_{T} f} \omega=\mathbf{i}_{\nabla_{T} f} \mathbf{t} \omega=0
$$

implies

$$
\left\langle\mathbf{i}_{\nabla_{T} f} \omega \mid \mathbf{i}_{n_{\sigma}} \omega\right\rangle_{\Lambda T_{\sigma}^{*} \Omega}=0
$$

The condition $\mathbf{t} \omega=0$ also gives

$$
\left\langle\mathbf{i}_{n_{\sigma}} \omega \mid \mathbf{i}_{n_{\sigma}} \omega\right\rangle=\langle\omega \mid \omega\rangle,
$$

which yields the result.

\section{Remark 2.3.}

If instead of the condition, $\mathbf{t} \omega=0$, we assume $\mathbf{n} \omega=0$, then the integral term on $\partial \Omega$ in formula (2.15) comes with the sign + .

\subsection{Tangential Dirichlet realization.}

In this subsection, we specify the self-adjoint realization of $\Delta_{f, h}^{(0)}$ in which we are interested. When $f=0$, it is known as the relative problem (see $[\mathrm{Gu}]$ and references therein). The good property of this self-adjoint realization, denoted by $\Delta_{f, h}^{D T}$ is that it coincides with the Dirichlet realization on 0 -forms and preserves the complex structure :

$$
\left(1+\Delta_{f, h}^{D T,(p+1)}\right)^{-1} d_{f, h}^{(p)}=d_{f, h}^{(p)}\left(1+\Delta_{f, h}^{D T,(p)}\right)^{-1}
$$

and

$$
\left(1+\Delta_{f, h}^{D T,(p-1)}\right)^{-1} d_{f, h}^{(p-1), *}=d_{f, h}^{(p-1), *}\left(1+\Delta_{f, h}^{D T,(p)}\right)^{-1},
$$

on the form domain of $\Delta_{f, h}^{D T,(p)}$.

The simplest self-adjoint realization is the Friedrichs extension $\Delta_{f, h}^{D}$, starting from $\mathcal{C}_{0}^{\infty}\left(\Omega ; \Lambda T^{*} \Omega\right)$, which leads, when $\Omega$ is regular, with the elliptic regularity property, to the domain $D\left(\Delta_{f, h}^{D}\right)=H_{0}^{1}\left(\Omega ; \Lambda T^{*} \Omega\right) \cap H^{2}\left(\Omega ; \Lambda T^{*} \Omega\right)$. The problem is that $d_{f, h}$ does not preserve this domain. We will see that it is more natural for our problem to impose Dirichlet boundary conditions only on the tangential components, while completing these conditions with conditions on the codifferential. Other classical self-adjoint extensions are 
possible with the same properties and we refer the reader to [Schw] and [CL] for details.

We introduce the space

$$
\Lambda^{p} H_{0, T}^{1}=H_{0, T}^{1}\left(\Omega ; \Lambda^{p} T^{*} \Omega\right)=\left\{\omega \in H^{1}\left(\Omega ; \Lambda^{p} T^{*} \Omega\right) ; \mathbf{t} \omega=0\right\} .
$$

In the case $p=0$, it coincides with the standard space $H_{0}^{1}(\Omega)$, while for $p \geq 1$ the condition says only that the form vanishes on $\partial \Omega$ when applied to tangential $p$-vectors. Since the boundary $\partial \Omega$ is assumed to be regular the space

$$
\Lambda^{p} \mathcal{C}_{0, T}^{\infty}=\mathcal{C}_{0, T}^{\infty}\left(\Omega ; \Lambda^{p} T^{*} \Omega\right)=\left\{\omega \in \mathcal{C}^{\infty}\left(\bar{\Omega}, \Lambda^{p} T^{*} \Omega\right) ; \mathbf{t} \omega=0\right\}
$$

is dense in $\Lambda^{p} H_{0, T}^{1}$. The next construction is a variant of known results in the case $f=0$ (see $[\mathrm{Schw}]$ ). We will use the notations

$$
\mathcal{D}_{f, h}(\omega, \eta)=\left\langle d_{f, h} \omega \mid d_{f, h} \eta\right\rangle_{\Lambda^{p+1} L^{2}}+\left\langle d_{f, h}^{*} \omega \mid d_{f, h}^{*} \eta\right\rangle_{\Lambda^{p-1} L^{2}}
$$

and

$$
\mathcal{D}_{f, h}(\omega)=\mathcal{D}_{f, h}(\omega, \omega)=\left\|d_{f, h} \omega\right\|_{\Lambda^{p+1} L^{2}}^{2}+\left\|d_{f, h}^{*} \omega\right\|_{\Lambda^{p-1} L^{2}}^{2}
$$

\section{Proposition 2.4.}

The non negative quadratic form $\omega \rightarrow \mathcal{D}_{f, h}(\omega)$ is closed on $\Lambda^{p} H_{0, T}^{1}$. The associated Friedrichs (self-adjoint) extension is denoted by $\Delta_{f, h}^{D T,(p)}$. Its domain is

$$
D\left(\Delta_{f, h}^{D T,(p)}\right)=\left\{u \in \Lambda^{p} H^{2} ; \quad \mathbf{t} \omega=0 \text { and } \mathbf{t} d_{f, h}^{*} \omega=0\right\},
$$

and we have

$$
\forall \omega \in D\left(\Delta_{f, h}^{D T,(p)}\right), \quad \Delta_{f, h}^{D T,(p)} \omega=\Delta_{f, h}^{(p)} \omega
$$

Proof.

First we observe that the space $\Lambda^{p} H_{0, T}^{1}$ is isomorphic to the direct sum

$$
\Lambda^{p} H_{0}^{1} \oplus \mathbf{n} \Lambda^{p} H^{1 / 2}\left(\partial \Omega ; \Lambda^{p} T^{*} \Omega\right)
$$

with continuous embedding. Since $\partial \Omega$ is regular, one can indeed construct a right inverse $R$ to the trace operator $\gamma_{0}: \Lambda^{p} H^{1} \rightarrow \Lambda^{p} H^{1 / 2}\left(\partial \Omega ; \Lambda^{p} T^{*} \Omega\right)$, so that any $u \in \Lambda^{p} H^{1}$ can be written as the sum

$$
u=\left(u-R \gamma_{0} u\right)+R \gamma_{0} u
$$


with $\left(u-R \gamma_{0} u\right) \in \Lambda^{p} H_{0}^{1}$. Once the operator $R$ is chosen, the previous decomposition gives an isomorphism $u \rightarrow\left(u-R \gamma_{0} u, \gamma_{0} u\right)$ from $\Lambda^{p} H_{0, T}^{1}$ to $\Lambda^{p} H_{0}^{1} \oplus \mathbf{n} \Lambda^{p} H^{1 / 2}\left(\partial \Omega ; \Lambda^{p} T^{*} \Omega\right)$. Hence its dual is the direct sum of $\Lambda^{p} H^{-1}$ and $\mathbf{n} \Lambda^{p} H^{-1 / 2}\left(\partial \Omega ; \Lambda^{p} T^{*} \Omega\right)$ :

$$
\left(\Lambda^{p} H_{0, T}^{1}\right)^{\prime}=\Lambda^{p} H^{-1} \oplus \mathbf{n} \Lambda^{p} H^{-1 / 2}\left(\partial \Omega ; \Lambda^{p} T^{*} \Omega\right) .
$$

We have to check that $\omega \mapsto \mathcal{D}_{f, h}^{(p)}(\omega)+C\|\omega\|_{\Lambda^{p} L^{2}}^{2}$ is equivalent to the square of the $\Lambda^{p} H^{1}$ norm on $\Lambda^{p} H_{0, T}^{1}$. By (2.3)-(2.6) this is equivalent to the same result for $f=0$ and $h=1$. This last case is known as Gaffney's inequality which is a consequence of the Weitzenböck formula (see [Schw], Corollary 2.1.6). Hence the identity

$$
\forall \eta \in \Lambda^{p} H_{0, T}^{1}, \quad \mathcal{D}_{f, h}^{(p)}(\eta, \omega)=\left\langle\eta, A^{(p)} \omega\right\rangle
$$

defines an isomorphism $A^{(p)}: \Lambda^{p} H_{0, T}^{1} \rightarrow\left(\Lambda^{p} H_{0, T}^{1}\right)^{\prime}$. The self-adjoint Friedrichs extension $\Delta_{f, h}^{D T,(p)}$ is then defined as the operator

$$
D\left(\Delta_{f, h}^{D T,(p)}\right)=\left\{\omega \in \Lambda^{p} H_{0, T}^{1}, A^{(p)} \omega \in \Lambda^{p} L^{2}\right\}, \quad \Delta_{f, h}^{D T,(p)} \omega=A^{(p)} \omega .
$$

It remains to identify this domain and the explicit action of $A^{(p)}$. If $\eta$ belongs to $D\left(\Delta_{f, h}^{D T,(p)}\right)$, we use first the Green formula (2.12) in order to get

$$
\forall \omega \in \Lambda^{p} \mathcal{C}_{0}^{\infty}, \quad\left\langle A^{(p)} \omega \mid \eta\right\rangle=\mathcal{D}_{f, h}^{(p)}(\omega, \eta)=\left\langle\Delta_{f, h}^{(p)} \omega \mid \eta\right\rangle
$$

The inequality

$$
\left|\mathcal{D}_{f, h}^{(p)}(\omega, \eta)\right| \leq C\|\omega\|_{\Lambda^{p} H^{1}}\|\eta\|_{\Lambda^{p} H^{1}}
$$

together with the density of $\Lambda^{p} \mathcal{C}_{0}^{\infty}$ in $\Lambda^{p} H_{0}^{1}$ implies that $\Delta_{f, h}^{(p)} \eta \in \mathcal{D}^{\prime}\left(\Omega ; \Lambda^{p} T^{*} \Omega\right)$ is indeed the $\Lambda^{p} H^{-1}$ component of $A^{(p)} \eta$.

Assume that $\omega$ belongs to $\Lambda^{p} H_{0, T}^{1} \cap \Lambda^{p} H^{2}$; then the Green formula (2.12) gives

$$
h \int_{\partial \Omega}\left(\mathbf{t} d_{f, h}^{(p-1), *} \omega\right) \wedge \star \mathbf{n} \bar{\eta}=\mathcal{D}^{(p)}(\omega, \eta)-\left\langle\Delta_{f, h}^{(p)} \omega \mid \eta\right\rangle_{\Lambda^{p} L^{2}}, \forall \eta \in \Lambda^{p} H_{0, T}^{1} .
$$

By density, one can define, for any $\omega$ in $\Lambda^{p} H_{0, T}^{1}$ such that $\Delta_{f, h}^{(p)} \omega \in \Lambda^{p} L^{2}$, a trace of $\mathbf{t} d_{f, h}^{*} \omega$ by the previous identity, observing that the right-hand side 
defines an antilinear continuous form with respect to $\eta$. With this generalized definition of $\mathbf{t} d_{f, h}^{(p)} \omega$ we obtain

$$
D\left(A^{(p)}\right)=\left\{\omega \in \Lambda_{0, T}^{p} H^{1}, \Delta_{f, h}^{(p)} \omega \in \Lambda^{p} L^{2} \text { and } \mathbf{t} d_{f, h}^{(p-1), *} \omega=0\right\} .
$$

The last point consists in observing that the boundary value problem

$$
\Delta_{f, h}^{(p)} u=g, \quad \mathbf{t} u=g_{1}, \quad \mathbf{t} d_{f, h}^{(p-1), *} u=g_{2}
$$

satisfies the Lopatinski-Shapiro conditions. At the principal symbol level ( $h>0$ fixed), these conditions are indeed the same as for

$$
\left(d d^{*}+d^{*} d\right)^{(p)} u=g, \quad \mathbf{t} u=g_{1}, \quad \mathbf{t} d^{(p-1), *} u=g_{2} .
$$

This is checked in $[\mathrm{Schw}]$. Hence any solution to (2.17) with $g \in \Lambda^{p} L^{2}$, $g_{1}=g_{2}=0$ belongs to $\Lambda^{p} H^{2}$.

\section{Proposition 2.5.}

For any $p \in\{0, \ldots, n\}$, the self-adjoint unbounded operator of domain $D\left(\Delta_{f, h}^{D T,(p)}\right)=\left\{\omega \in \Lambda H^{2}, \mathbf{t} \omega=0, \mathbf{t} d_{f, h}^{(p-1), *} \omega=0\right\}$ and defined by

$$
\Delta_{f, h}^{D T,(p)} \omega=\Delta_{f, h}^{(p)} \omega, \forall \omega \in D\left(\Delta_{f, h}^{D T,(p)}\right),
$$

has a compact resolvent.

Moreover, if $z \in \mathbb{C} \backslash \mathbb{R}_{+}$, the commutation relations

$$
\begin{gathered}
\left(z-\Delta_{f, h}^{D T,(p+1)}\right)^{-1} d_{f, h}^{(p)} v=d_{f, h}^{(p)}\left(z-\Delta_{f, h}^{D T,(p)}\right)^{-1} v, \\
\text { and } \\
\left(z-\Delta_{f, h}^{D T,(p-1)}\right)^{-1} d_{f, h}^{(p-1), *} v=d_{f, h}^{(p-1), *}\left(z-\Delta_{f, h}^{D T,(p)}\right)^{-1} v,
\end{gathered}
$$

hold for any $v \in \Lambda^{p} H_{0, T}^{1}$.

Proof.

The domain of the operator is contained in $\Lambda^{p} H^{2}$, which is compactly embedded in $\Lambda^{p} L^{2}$. This yields the first statement.

Since $\Lambda^{p} \mathcal{C}_{0, T}^{\infty}$ is dense in $\Lambda^{p} H_{0, T}^{1}$, it is sufficient to consider the case when $v \in \Lambda^{p} \mathcal{C}_{0, T}^{\infty}$. For such a $v$ and for $z \in \mathbb{C} \backslash \mathbb{R}_{+}$, we set

$$
u=\left(z-\Delta_{f, h}^{D T,(p)}\right)^{-1} v
$$


Due to the ellipticity of the associated boundary problem (the LopatinskiShapiro conditions are verified) $u$ belongs to $\mathcal{C}^{\infty}\left(\bar{\Omega} ; \Lambda^{p} T^{*} \Omega\right)$. The commutation relations $(2.1)$ and $(2.2)$ can be applied since here $f \in \mathcal{C}^{\infty}(\bar{\Omega} ; \mathbb{R})$ :

$$
\left(z-\Delta_{f, h}^{(p+1)}\right) d_{f, h}^{(p)} u=d_{f, h}^{(p)}\left(z-\Delta_{f, h}^{(p)}\right) u=d_{f, h}^{(p)} v
$$

and

$$
\left(z-\Delta_{f, h}^{(p-1)}\right) d_{f, h}^{(p-1), *} u=d_{f, h}^{(p-1), *}\left(z-\Delta_{f, h}^{(p)}\right) u=d_{f, h}^{(p-1), *} v .
$$

Since $u \in D\left(\Delta_{f, h}^{D T,(p)}\right)$, we have $\mathbf{t} u=0$ and $\mathbf{t} d_{f, h}^{*} u=0$. Since $\mathbf{t}$ commute with the differential, we get

$$
\mathbf{t} d_{f, h} u=h d \mathbf{t} u+(\mathbf{t} d f) \wedge(\mathbf{t} u)=0 .
$$

For the tangential trace of the codifferential, we write

$$
\mathbf{t} d_{f, h}^{*}\left(d_{f, h} u\right)=z \mathbf{t} u-\mathbf{t} v-\mathbf{t} d_{f, h} d_{f, h}^{*} u=z \mathbf{t} u-\mathbf{t} v-d_{f, h} \mathbf{t} d_{f, h}^{*} u=0 .
$$

Hence $d_{f, h}^{(p)} u$ belongs to $D\left(\Delta_{f, h}^{D T,(p+1)}\right)$ and the identity (2.18) yields

$$
d_{f, h}^{(p)}\left(z-\Delta_{f, h}^{D T,(p)}\right)^{-1} v=d_{f, h} u=\left(z-\Delta_{f, h}^{D T,(p+1)}\right)^{-1} d_{f, h} v,
$$

which proves the first announced commutation relation.

For the second one, the verification that $d_{f, h}^{(p-1), *} u$ belongs to $D\left(\Delta_{f, h}^{D T,(p-1)}\right)$ is even simpler. First the property, $\mathbf{t} d_{f, h}^{(p-1){ }^{*} *} u=0$, is given by $u \in D\left(\Delta_{f, h}^{D T,(p)}\right)$; then $\mathbf{t} d_{f, h}^{*}\left(d_{f, h}^{*} u\right)=\mathbf{t} 0=0$. We obtain

$$
d_{f, h}^{(p-1), *}\left(z-\Delta_{f, h}^{D T,(p)}\right)^{-1} v=d_{f, h}^{(p-1), *} u=\left(z-\Delta_{f, h}^{D T,(p-1)}\right)^{-1} d_{f, h}^{(p-1), *} v .
$$

\section{Remark 2.6.}

Note that the above commutation relations cannot be extended to $v \in \Lambda^{p} H^{1}$. Assume for example that $v$ is $\mathcal{C}^{\infty}$ up to the boundary. Starting from the identity

$$
v=\left(z-\Delta_{f, h}^{D T,(p)}\right)\left(z-\Delta_{f, h}^{D T,(p)}\right)^{-1} v
$$

we can write

$$
\begin{aligned}
v= & z\left(z-\Delta_{f, h}^{D T,(p)}\right)^{-1} v-d_{f, h}^{(p), *} d_{f, h}^{(p)}\left(z-\Delta_{f, h}^{D T,(p)}\right)^{-1} v \\
& -d_{f, h}^{(p-1)} d_{f, h}^{(p-1), *}\left(z-\Delta_{f, h}^{D T,(p)}\right)^{-1} v \\
= & z u_{1}-d_{f, h}^{(p), *} u_{2}-d_{f, h}^{(p-1)} u_{3}
\end{aligned}
$$


with the relations $u_{1} \in D\left(\Delta_{f, h}^{D T,(p)}\right), \quad u_{2}=d_{f, h}^{(p)}\left(z-\Delta_{f, h}^{D T,(p)}\right)^{-1} v$, and $u_{3}=d_{f, h}^{(p-1), *}\left(z-\Delta_{f, h}^{D T,(p)}\right)^{-1} v$.

Now the commutation relations would imply $u_{2} \in D\left(\Delta_{f, h}^{D T,(p+1)}\right)$ and $u_{3} \in D\left(\Delta_{f, h}^{D T,(p-1)}\right)$.

The form $v$ should then satisfy on the boundary

$$
\mathbf{t} v=z \mathbf{t} u_{1}-\mathbf{t} d_{f, h}^{(p), *} u_{2}-d_{f, h}^{(p-1)} \mathbf{t} u_{3}=0 .
$$

From Proposition 2.5 and Stone's Formula we deduce the

\section{Corollary 2.7.}

For any Borel subset $E \subset \mathbb{R}$, the identities

$$
1_{E}\left(\Delta_{f, h}^{D T,(p+1)}\right) d_{f, h}^{(p)} v=d_{f, h}^{(p)} 1_{E}\left(\Delta_{f, h}^{D T,(p)}\right) v
$$

and

$$
1_{E}\left(\Delta_{f, h}^{D T,(p-1)}\right) d_{f, h}^{(p-1), *} v=d_{f, h}^{(p-1), *} 1_{E}\left(\Delta_{f, h}^{D T,(p)}\right) v
$$

hold for all $v \in \Lambda^{p} H_{0, T}^{1}$.

In particular, if $v$ is an eigenvector of $\Delta_{f, h}^{D T,(p)}$ corresponding to the eigenvalue $\lambda$, then $d_{f, h}^{(p)} v\left(\right.$ resp. $d_{f, h}^{(p-1), *} v$ ) belongs to the spectral subspace $\operatorname{Ran} 1_{\{\lambda\}}\left(\Delta_{f, h}^{D T,(p+1)}\right)$ (resp. Ran $\left.1_{\{\lambda\}}\left(\Delta_{f, h}^{D T,(p-1)}\right)\right)$.

Proposition 2.5 and Corollary 2.7 were stated for $p$-forms $v \in \Lambda^{p} H_{0, T}^{1}(\Omega)$, belonging to the form domain of $\Delta_{f, h}^{D T,(p)}$. It is convenient to work in this framework because the multiplication by any cut-off function preserves $\Lambda H_{0, T}^{1}(\Omega)$ :

$$
\left(\omega \in \Lambda H_{0, T}^{1}(\Omega), \quad \chi \in \mathcal{C}^{\infty}(\bar{\Omega})\right) \Rightarrow\left(\chi \omega \in \Lambda H_{0, T}^{1}(\Omega)\right),
$$

while this property is no more true for $D\left(\Delta_{f, h}^{D T}\right)$. In this spirit, we will often refer to the next easy consequence of the spectral theorem.

\section{Lemma 2.8.}

Let $A$ be a nonnegative self-adjoint operator on a Hilbert space $\mathcal{H}$ given as the Friedrichs extension of a closed quadratic form $q_{A}$ with form domain $Q(A)$. Then for any $a, b \in(0,+\infty)$, the implication

$$
\left(q_{A}(u) \leq a\right) \Rightarrow\left(\left\|1_{[b,+\infty)}(A) u\right\|^{2} \leq \frac{a}{b}\right)
$$

holds for any $u \in Q(A)$. 


\subsection{Boundary reduced Witten complex}

We end this section with the introduction of the reduced complex which is standard in the boundaryless case since Witten (see [Wit], [Sim2], [CFKS], [HelSj4] and the book [Zh]). This will motivate the preliminary analysis given in the next section.

Let us assume that the dimension $m_{p}^{\bar{\Omega}}$ of $F^{(p)}=\operatorname{Ran} 1_{\left[0, h^{3 / 2}\right)}\left(\Delta_{f, h}^{D T,(p)}\right)$ is independent of $h \in\left(0, h_{0}\right)$ for $h_{0}>0$ small enough. The previous proposition says that $\beta_{f, h}^{(p)}=\left.d_{f, h}^{(p)}\right|_{F^{(p)}}$ and $\beta_{f, h}^{(p), *}=\left.d_{f, h}^{(p-1), *}\right|_{F^{(p)}}(p=0, \ldots, n)$ define two complexes of finite dimensional spaces :

$$
\begin{array}{ll}
0 \longrightarrow F^{(0)} \stackrel{\beta_{f, h}^{(0)}}{\longrightarrow} F^{(1)} \stackrel{\beta_{f, h}^{(1)}}{\longrightarrow} \ldots & \stackrel{\beta_{f, h}^{(n-1)}}{\longrightarrow} F^{(n)} \rightarrow 0 \\
0 \leftarrow F^{(0)} \stackrel{\beta_{f, h}^{(0), *}}{\longleftarrow} F^{(1)} \stackrel{\beta_{f, h}^{(1), *}}{\longleftarrow} \ldots & \stackrel{\beta_{f, h}^{(n-1), *}}{\longleftarrow} F^{(n)} \leftarrow 0 .
\end{array}
$$

If $b_{p}^{\bar{\Omega}}, p \in\{0, \ldots, n\}$, denote the Betti numbers of the $\beta_{f, h}^{(p)}$ complex, then the polynomials,

$$
M(X)=\sum_{p=0}^{n} m_{p}^{\bar{\Omega}} X^{p} \quad \text { and } \quad B(X)=\sum_{p=0}^{n} b_{p}^{\bar{\Omega}} X^{p}
$$

satisfy

$$
M(X)-B(X)=(1+X) Q(X),
$$

where the polynomial $Q(X)$ has non negative coefficients.

In the boundaryless case, the numbers $m_{p}$ are exactly the number of critical points with index $p$ and this is the core of Witten's approach to Morse inequalities. In the boundary case, it is no more true. The next section explains the role of the boundary conditions on the spaces $F^{(p)}$.

\section{First localization of the spectrum.}

\subsection{Introduction}

In this section, we check that the number of eigenvalues of $\Delta_{f, h}^{D T,(p)}$ smaller than $h^{3 / 2}$ equals a Morse index $m_{p}^{\bar{\Omega}}$ which involves in its definition the boundary condition. For this we need a first localization of the eigenvectors. Although the results presented here are closely related to those of [CL], we need 
additional information and technical analysis for the following reason :

When one is interested only in the Morse theory the metric plays no relevant role and it is possible, without loss of generality, to assume that it has a simple form at the critical points. This simplification, which leads to a much easier analysis, was used by many authors [CFKS], [CL], [Bis], [Bur], and [He1]. Since we are interested in quantitative results with a prescribed metric from the beginning, the dependence with respect to the metric has to be analyzed carefully. One difficulty comes from the fact that the boundary condition and therefore the domain of $\Delta_{f, h}^{(p)}$ depends on the metric $g$.

\subsection{Morse-Witten theory for boundary value problems.}

In order to make the connection between the tangential Dirichlet realization of the Witten Laplacian $\Delta_{f, h}^{D T}$ and the Morse theory, we assume additional properties for the function $f$ up to the boundary $\partial \Omega$.

\section{Assumption 3.1.}

The real valued function $f \in \mathcal{C}^{\infty}(\bar{\Omega})$ is a Morse function on $\Omega$ with no critical points in $\partial \Omega$. In addition its restriction $\left.f\right|_{\partial \Omega}$ is a Morse function on $\partial \Omega$.

With this assumption, the function $f$ admits a finite number $m_{p}^{\Omega}$ of critical points with index $p$ in $\Omega$. Those numbers have to be modified for the boundary problem according to [CL] in order to take into account eigenvectors which possibly concentrate $($ as $h \rightarrow 0$ ) on $\partial \Omega$. Note first that the assumption that there is no critical point on $\partial \Omega$ implies that the outgoing normal derivative $\partial_{n} f(U)$ is not 0 , if $U$ is a critical point of $\left.f\right|_{\partial \Omega}$.

\section{Definition 3.2.}

For $\ell \in\{0, \ldots, n-1\}$, the integer $m_{\ell,+}^{\partial \Omega}$ is the number of critical points $U$ of $\left.f\right|_{\partial \Omega}$ with index $\ell$ such that $\partial_{n} f(U)>0$ (with the additional convention $\left.m_{-1,+}^{\partial \Omega}=0\right)$.

For $p \in\{0, \ldots, n\}$, the integer $m_{p}^{\bar{\Omega}}$ is defined as

$$
m_{p}^{\bar{\Omega}}=m_{p}^{\Omega}+m_{p-1,+}^{\partial \Omega} .
$$

We will prove the

\section{Theorem 3.3.}

Under Assumption 3.1, there exists $h_{0}>0$, such that the tangential Dirichlet 
realization of the Witten Laplacian $\Delta_{f, h}^{D T}$ introduced in Subsection 2.4 has, for $h \in\left(0, h_{0}\right]$, the following property :

For any $p \in\{0, \ldots, n\}$, the spectral subspace $F^{(p)}=\operatorname{Ran}_{\left[0, h^{3 / 2}\right)}\left(\Delta_{f, h}^{D T,(p)}\right)$ has rank : $\operatorname{dim} F^{(p)}=m_{p}^{\bar{\Omega}}$.

Moreover the Betti numbers $b_{p}^{\bar{\Omega}}$ are homotopy invariants and satisfy the homological relations $(2.22)$.

\section{Remark 3.4.}

a) The role of the condition $\partial_{n} f(U)>0$ can be easily understood by considering the one-dimensional problem with $f(x)=x$ on the interval $[0,1]$. On 0 -forms, $\Delta_{f, h}^{D T,(0)}$ corresponds to a Dirichlet realization, while $\Delta_{f, h}^{D T,(1)}$ corresponds to a realization with an $h$-dependent Robin boundary condition $h \partial_{x} u-\left(\partial_{x} f\right) u=0$, where the function $u(x)$ has to be identified with the 1-form $u(x) d x$.

b) With the normal boundary conditions $\mathbf{n} \omega=0$ and $\mathbf{n} d_{f, h} \omega=0$, the number $m_{p-1,+}^{\partial \Omega}$ has to be replaced by $m_{p-1,-}^{\partial \Omega}$, which corresponds to the condition $\partial_{n} f(U)<0($ see $[C L])$.

We shall use a similar technique to the one presented in [Sim2], [CFKS] and $[\mathrm{CL}]$ by making rather rough estimates in terms of quadratic forms. We first consider a model half-space problem which permits, after a careful treatment of the metric, to separate tangential and normal coordinates. The localization process and the proof of Theorem 3.3 will be achieved in Subsection 3.4.

\subsection{A model half-space problem.}

We consider in this subsection a half-space model problem which will be used in the localization of the eigenvectors of $\Delta_{f, h}^{D T}$ on $\bar{\Omega}$ and will provide quasimodes.

We start first with some results on $\mathbb{R}^{k}$, which will be applied later with $k=n-1$.

\subsubsection{Witten Laplacian on $\mathbb{R}^{k}$ with one low-lying eigenvalue.}

The metric $g$ on $\mathbb{R}^{k}$ is a $\mathcal{C}^{\infty}$ metric which equals the euclidean metric outside a compact set $K$. 


\section{Assumption 3.5.}

The function $f$ is a Morse $\mathcal{C}^{\infty}$ real valued function and there exist $C_{1}>0$ and a compact $K$ such that :

$$
\forall x \in \mathbb{R}^{k} \backslash K, \quad|\nabla f(x)| \geq C_{1}^{-1} \quad \text { and } \quad|\operatorname{Hess} f(x)| \leq C_{1}|\nabla f(x)|^{2} .
$$

Note that the above assumption ensures that $f$ admits a finite number of critical points and $m_{p}$ will denote the number of critical points with index $p$.

\section{Proposition 3.6.}

Under Assumption 3.5, there exist $h_{0}>0, c_{0}>0$ and $c_{1}>0$ such that the following properties are satisfied for any $h \in\left(0, h_{0}\right]$ :

i) The Witten Laplacians $\Delta_{f, h}$ as an unbounded operator on $L^{2}\left(\mathbb{R}^{k} ; \Lambda T^{*} \mathbb{R}^{k}\right)$ is essentially self-adjoint on $\mathcal{C}_{0}^{\infty}\left(\mathbb{R}^{k} ; \Lambda T^{*} \mathbb{R}^{k}\right)$.

ii) For any Borel subset $E$ in $\mathbb{R}$, the identities,

$$
\text { and } \begin{aligned}
1_{E}\left(\Delta_{f, h}^{(p+1)}\right) d_{f, h}^{(p)} u & =d_{f, h}^{(p)} 1_{E}\left(\Delta_{f, h}^{(p)}\right) u, \\
1_{E}\left(\Delta_{f, h}^{(p-1)}\right) d_{f, h}^{(p), *} u & =d_{f, h}^{(p), *} 1_{E}\left(\Delta_{f, h}^{(p)}\right) u
\end{aligned}
$$

hold, for any $u$ belonging to the form domain of $\Delta_{f, h}^{(p)}$.

In particular, if $v$ is an eigenvector of $\Delta_{f, h}^{(p)}$ associated with the eigenvalue $\lambda$, then $d_{f, h}^{(p)} v$ (resp. $d_{f, h}^{(p-1), *} v$ ) belongs to the spectral subspace $\operatorname{Ran} 1_{\{\lambda\}}\left(\Delta_{f, h}^{(p+1)}\right)$ (resp. Ran $1_{\{\lambda\}}\left(\Delta_{f, h}^{(p-1)}\right)$ ).

iii) The essential spectrum $\sigma_{\text {ess }}\left(\Delta_{f, h}^{(p)}\right)$ is contained in $\left[c_{1},+\infty\right)$.

$i v)$ The range of the spectral projection $1_{\left[0, c_{0} h\right)}\left(\Delta_{f, h}^{(p)}\right)$ has dimension $m_{p}$, for all $h \in\left(0, h_{0}\right]$.

Proof.

We give the proof for the sake of completeness (see also [Jo]).

i) The operator

$$
\Delta_{f, h}=-h^{2} \Delta+\Psi(x)=d_{f, h} d_{f, h}^{*}+d_{f, h}^{*} d_{f, h}
$$

is non-negative on $\mathcal{C}_{0}^{\infty}\left(\mathbb{R}^{k} ; \Lambda T^{*} \mathbb{R}^{k}\right)$ and the matrix-valued function $\Psi(x)$ is $\mathcal{C}^{\infty}$. By Simader's result (see [Sima], [He3]), $\Delta_{f, h}$ is essentially self-adjoint on $\mathcal{C}_{0}^{\infty}\left(\mathbb{R}^{n-1} ; \Lambda T^{*} \mathbb{R}^{k}\right)$.

ii) The proof is the same as in Proposition 2.5 and Corollary 2.7 with 
$\Lambda^{p} \mathcal{C}_{0, T}^{\infty}(\Omega)$ replaced by $\Lambda^{p} \mathcal{C}_{0}^{\infty}\left(\mathbb{R}^{k}\right)$. By i $), \Lambda^{p} \mathcal{C}_{0}^{\infty}\left(\mathbb{R}^{k}\right)$ is dense in $D\left(\Delta_{f, h}^{(p)}\right)$ and therefore in the form domain of $\Delta_{f, h}^{(p)}$.

iii) The localization of the essential spectrum is a consequence of our assumptions which imply the existence of $C>0$ such that, for all $u \in \Lambda^{p} \mathcal{C}_{0}^{\infty}(\complement K)$,

$$
\left\langle u \mid \Delta_{f, h}^{(p)} u\right\rangle \geq\left\langle u \mid \Delta_{0, h}^{(p)} u\right\rangle+\frac{1}{C}\|u\|^{2}-C h\|u\|^{2} .
$$

When $h<h_{0}$, with $h_{0}=\frac{1}{2 C_{1}^{2}}$, we get

$$
\left\langle u \mid \Delta_{f, h}^{(p)} u\right\rangle \geq \frac{1}{2 C}\|u\|^{2}, \forall u \in \Lambda^{p} C_{0}^{\infty}(\complement K),
$$

and iii) by using Persson's Lemma.

iv) The previous inequality combined with a simple partition of unity argument shows that any normalized eigenvector $\psi_{h}$ associated with an eigenvalue $\lambda_{h}$ in $\left[0, c_{0} h\right)$ of $\Delta_{f, h}^{(p)}$ is localized in a neighborhood of $K$. Take indeed $\chi_{i} \in \mathcal{C}^{\infty}\left(\mathbb{R}^{k}\right), i=1,2$, such that $\chi_{1} \in \mathcal{C}_{0}^{\infty}\left(\mathbb{R}^{k}\right), \chi_{1}=1$ in a neighborhood of $K, \chi_{1}^{2}+\chi_{2}^{2}=1$, and write :

$$
\lambda_{h}|| \psi_{h}\left\|^{2}=\left\langle\chi_{1} \psi_{h} \mid \Delta_{f, h}^{(p)} \chi_{1} \psi_{h}\right\rangle+\left\langle\chi_{2} \psi_{h} \mid \Delta_{f, h}^{(p)} \chi_{2} \psi_{h}\right\rangle-h^{2} \sum_{i=1,2}\right\| \nabla \chi_{i} \psi_{h} \|^{2} .
$$

This leads, for $h$ small enough, to

$$
\begin{aligned}
& \left\|\chi_{2} \psi_{h}\right\|^{2} \leq 2 C \lambda_{h}+2 C\left(\sum_{i=1,2} \max _{x \in K}\left|\nabla \chi_{i}(x)\right|^{2}\right) h^{2} \leq 4 C c_{0} h, \\
& 1=\left\|\psi_{h}\right\| \leq\left(1+C^{\prime} h\right)\left\|\chi_{1} \psi_{h}\right\|,
\end{aligned}
$$

and

$$
\left\langle\chi_{1} \psi_{h} \mid \Delta_{f, h}^{(p)} \chi_{1} \psi_{h}\right\rangle \leq C^{\prime \prime} h^{2}+c_{0} h \leq 2 c_{0} h\left\|\psi_{h}\right\|^{2} \leq C^{\prime \prime \prime} c_{0} h\left\|\chi_{1} \psi_{h}\right\|^{2} .
$$

Hence the problem is reduced to the case of a boundaryless compact manifold presented in $[\mathrm{CFKS}]$ and $[\mathrm{HelSj} 4]$. With $c_{0}>0$ small enough, their related results, which rely here on harmonic approximations around the critical points of $f$, and the two previous estimates imply that $\psi_{h}$ has to lie within a distance less than $1 / 2$ from a finite dimensional space with dimension $m_{p}$. This yields

$$
\operatorname{dim} \operatorname{Ran} 1_{\left[0, c_{0} h\right)}\left(\Delta_{f, h}^{(p)}\right)=m_{p}
$$


We will need the following version of those results in the specific case when $f$ admits a unique critical point with index $p_{0}$.

\section{Proposition 3.7.}

If the Morse function $f$ satisfies Assumption 3.5 and admits a unique critical point at $x=0$ with index $p_{0}$, so $m_{p}=\delta_{p, p_{0}}$, then there exist $h_{0}>0$ and $c_{0}>0$, such that the following properties hold for $h \in\left(0, h_{0}\right]$ :

i) For $p \neq p_{0}, \Delta_{f, h}^{(p)} \geq c_{0} h \mathrm{Id}$.

ii) If $\psi_{p_{0}}^{h}$ is a normalized eigenvector of the one dimensional spectral subspace $\operatorname{Ran} 1_{\left[0, c_{0} h\right)}\left(\Delta_{f, h}^{\left(p_{0}\right)}\right)$, it satisfies

$$
d_{f, h} \psi_{p_{0}}^{h}=0, \quad d_{f, h}^{\left(p_{0}-1\right), *} \psi_{p_{0}}^{h}=0 \quad \text { and } \quad \Delta_{f, h}^{\left(p_{0}\right)} \psi_{p_{0}}^{h}=0,
$$

so that $\operatorname{Ran} 1_{\left[0, c_{0} h\right)}\left(\Delta_{f, h}^{\left(p_{0}\right)}\right)=\operatorname{Ker} \Delta_{f, h}^{\left(p_{0}\right)}$. Moreover

$$
\sigma\left(\Delta_{f, h}^{\left(p_{0}\right)}\right) \backslash\{0\} \subset\left[c_{0} h, \infty\right) .
$$

iii) If $\chi \in \mathcal{C}_{0}^{\infty}\left(\mathbb{R}^{k}\right)$ satisfies $\chi=1$ in a neighborhood of 0 , then there exists $C_{\chi} \geq 1$, such that, for all $h \in\left(0, h_{0} / C_{\chi}\right)$, the inequality,

$$
(1-\chi) \Delta_{f, h}^{(p)}(1-\chi) \geq C_{\chi}^{-1}[1-\chi]^{2}
$$

holds in the sense of quadratic form on $\Lambda^{p} H^{1}\left(\mathbb{R}^{k}\right)$.

Proof.

One uses first for i) the property that : dim $\operatorname{Ran} 1_{\left[0, c_{0} h\right)}\left(\Delta_{f, h}^{(p)}\right)=m_{p}$. Let us now show ii). Assume that $\psi_{p_{0}}^{h}$ is an eigenvector of $\Delta_{f, h}^{\left(p_{0}\right)}$ with eigenvalue $\lambda_{h} \in\left[0, c_{0} h\right)$. If $d_{f, h}^{\left(p_{0}\right)} \psi_{p_{0}}^{h}$ was not 0 , it would be an eigenvector of $\Delta_{f, h}^{\left(p_{0}+1\right)}$ with eigenvalue $\lambda_{h} \in\left[0, c_{0} h\right)$. Hence $d_{f, h} \psi_{p_{0}}^{h}=0$ and similarly $d_{f, h}^{\left(p_{0}-1\right), *} \psi_{p_{0}}^{h}=0$. This implies $\lambda_{h}=0$.

For iii), we note that

$$
\Delta_{f, h}^{(p)} \geq|\nabla f(x)|^{2}(1-C h),
$$

with $|\nabla f(x)|^{2} \geq c_{\chi}>0$ for $x \notin \operatorname{supp} \chi$.

This implies

$$
(1-\chi) \Delta_{f, h}^{(p)}(1-\chi) \geq c_{\chi}(1-C h)_{+}[1-\chi]^{2},
$$

for $h \in\left(0, h_{0}\right)$ and the result holds for $C_{\chi}>0$ large enough and $h_{0}$ small enough. 


\subsubsection{Small eigenvalues of the half space problems.}

We work here on $\mathbb{R}_{-}^{n}=\mathbb{R}^{n-1} \times(-\infty, 0)$. We assume that there are coordinates $\left(x^{\prime}, x_{n}\right)$ such that the metric $g=\sum_{i, j=1}^{n} g_{i j}(x) d x_{i} d x_{j}$ satisfies

$$
g_{i, n}=g_{n, i}=0 \quad \text { for } i<n
$$

and

$$
\forall x \in \overline{\mathbb{R}_{-}^{n}} \backslash K_{1}, \quad \partial_{x} g_{i j}(x)=0,
$$

for some compact set $K_{1} \subset \overline{\mathbb{R}_{-}^{n}}$. In this paragraph, the coordinates $\left(x, x_{n}\right)$ are fixed while different metrics on $\overline{\mathbb{R}_{-}^{n}}$ are considered. The notation $G(\cdot)$ will be used for the matrix valued map $x \mapsto G(x)={ }^{t} G(x)=\left(g_{i j}(x)\right)_{i, j} \in G L^{n}(\mathbb{R})$, which is assumed to be a $\mathcal{C}^{\infty}$ function. According to the standard notation, the coefficients of $G(x)^{-1}$ are written $g^{i j}(x)$.

We also consider a function $f$ which has a specific form in the same coordinates $\left(x^{\prime}, x_{n}\right)$.

\section{Assumption 3.8.}

The function $f \in \mathcal{C}^{\infty}\left(\overline{\mathbb{R}_{-}^{n}}\right)$ satisfies :

i) The estimates $|\nabla f(x)| \geq C^{-1} \quad$ and $\quad\left|\partial_{x}^{\alpha} f(x)\right| \leq C_{\alpha}$ hold, for all $x \in \overline{\mathbb{R}_{-}^{n}}$ and all $\alpha \in \mathbb{N}^{d}, \alpha \neq 0$.

ii) The function $f$ is the sum $f\left(x^{\prime}, x_{n}\right)=-\frac{1}{2} f_{+}\left(x_{n}\right)+\frac{1}{2} f_{-}\left(x^{\prime}\right)$, where there exists $C_{1}>0$ such that:

$$
C_{1}^{-1} \leq\left|\partial_{x_{n}} f_{+}\left(x_{n}\right)\right| \leq C_{1}
$$

and where $f_{-}$is a Morse function on $\mathbb{R}^{n-1}$, which satisfies Assumption 3.5 for the metric $\sum_{i, j=1}^{n-1} g_{i j}\left(x^{\prime}, 0\right) d x_{i} d x_{j}$ and admits a unique critical point at $x^{\prime}=0$ with index $p_{0}$.

The boundedness of $\left|\partial_{x}^{\alpha} f\right|, 1 \leq|\alpha| \leq 2$, avoids any subtle questions about the domains.

\section{Proposition 3.9.}

Under Assumption 3.8-i), the unbounded operator $\Delta_{f, h}^{D T}$ on $L^{2}\left(\mathbb{R}_{-}^{n} ; \Lambda T^{*} \mathbb{R}_{-}^{n}\right)$, with domain

$$
D\left(\Delta_{f, h}^{D T}\right)=\left\{\omega \in \Lambda H^{2}\left(\mathbb{R}_{-}^{n}\right), \quad \mathbf{t} \omega=0, \quad \mathbf{t} d_{f, h}^{*} \omega=0\right\},
$$


is self-adjoint.

If $E$ is any Borel subset of $\mathbb{R}$, the relations

$$
\text { and } \begin{aligned}
1_{E}\left(\Delta_{f, h}^{D T,(p+1)}\right) d_{f, h}^{(p)} u & =d_{f, h}^{(p)} 1_{E}\left(\Delta_{f, h}^{D T,(p)}\right) u \\
1_{E}\left(\Delta_{f, h}^{D T,(p-1)}\right) d_{f, h}^{(p), *} u & =d_{f, h}^{(p), *} 1_{E}\left(\Delta_{f, h}^{D T,(p)}\right) u
\end{aligned}
$$

hold for any $u \in \Lambda^{p} H_{0, T}^{1}\left(\mathbb{R}_{-}^{n}\right)$.

Proof.

The uniform estimate on $\nabla f$ permits the same proof as for Proposition 2.5 and Corollary 2.7 (Here $\mathcal{C}_{0, T}^{\infty}$ denotes the space of $\mathcal{C}^{\infty}$ compactly supported functions in $\overline{\mathbb{R}_{-}^{n}}$ with a vanishing tangential component on $\left\{x_{n}=0\right\}$ ).

We are looking for a result similar to Proposition 3.7 for the boundaryless case. One difficulty here comes from the metric which, although diagonal in the coordinates $\left(x^{\prime}, x_{n}\right)$, is not constant. The general case can be reduced to a simpler situation where $g_{i j}(x)=g_{i j}\left(x^{\prime}\right)$ with $g_{n n}=1$ after several steps. We need some notations.

\section{Definition 3.10.}

For a metric $g$ which satisfies (3.5), the corresponding $H^{s}$-norm on $\Lambda^{p} H^{s}\left(\overline{\mathbb{R}_{-}^{n}}\right)$ is denoted by \|\|$_{\Lambda^{p} H^{s}, g}$ and the notation \|\|$_{\Lambda^{p} H^{s}}$ is kept for the euclidean metric $g_{e}=\sum_{i=1}^{n} d x_{i}^{2}$.

Similarly, the quadratic form associated with $\Delta_{f, h}^{D T,(p)}$ is written

$$
\mathcal{D}_{g, f, h}(\omega)=\left\|d_{f, h}^{*, g} \omega\right\|_{\Lambda^{p-1} L^{2}, g}^{2}+\left\|d_{f, h} \omega\right\|_{\Lambda^{p+1} L^{2}, g}^{2}, \quad \forall \omega \in \Lambda^{p} H_{0, T}^{1}\left(\mathbb{R}_{-}^{n}\right)
$$

where the codifferential $d_{f, h}^{*, g}$ also depends on $g$.

$A \mathcal{K}$-set is a set of metrics $g$ which satisfy the conditions (3.5) and which is compact for the $\mathcal{C}^{\infty}\left(K_{1}\right)$-topology.

A $\mathcal{K}$-set is a set of metrics $g$ which satisfy the conditions (3.5) and so that $G(x)$ and $G(x)^{-1}$ are bounded in the $\mathcal{C}^{\infty}\left(K_{1}\right)$-topology. Note that, when the metric $g$ lies in a fixed $\mathcal{K}$-set $(h=1)$, the $H^{s}$-norms are uniformly equivalent to the norm associated with the euclidean metric $g_{e}$. The required accuracy while comparing the quadratic forms $\mathcal{D}_{g, f, h}$ needs some care.

The first result provides a reduction to the case $\partial_{x_{n}} G=0$. 


\section{Lemma 3.11.}

Let $g_{1}$ and $g_{2}$ be two metrics which satisfy (3.5) and coincide on $\left\{x_{n}=0\right\}$. Let $f$ be a function satisfying Assumption 3.8. There exist constants $C_{12} \geq 1$ and $h_{0}>0$ such that the inequality,

$$
\mathcal{D}_{g_{2}, f, h}(\omega) \geq\left(1-C_{12} h^{2 / 5}\right) \mathcal{D}_{g_{1}, f, h}(\omega)-C_{12} h^{7 / 5}\|\omega\|_{\Lambda^{p} L^{2}, g_{1}}^{2}
$$

holds for $\omega \in \Lambda^{p} H_{0, T}^{1}\left(\mathbb{R}_{-}^{n}\right)$, with $p \in\{0, \ldots, n\}$ and $h \in\left(0, h_{0}\right)$, as soon as $\operatorname{supp} \omega \subset\left\{x_{n} \geq-C_{0} h^{2 / 5}\right\}$.

Proof.

The matrices $G_{1}(x)$ and $G_{2}(x)$ associated with $g_{1}$ and $g_{2}$ in the coordinates $\left(x^{\prime}, x_{n}\right)$ satisfy the estimates

$$
\left|G_{1}(x)^{-1} G_{2}(x)-\operatorname{Id}_{\mathbb{R}^{n}}\right| \leq C h^{2 / 5}
$$

for all $x \in\left\{x_{n} \geq-C_{0} h^{2 / 5}\right\}$. Hence, for any differential form $\eta \in \Lambda L^{2}$ supported in $\left\{x_{n} \geq-C_{0} h^{2 / 5}\right\}$, the two $L^{2}$-norms differ by

$$
\left|\|\eta\|_{\Lambda L^{2}, g_{1}}^{2}-\|\eta\|_{\Lambda L^{2}, g_{2}}^{2}\right| \leq C h^{2 / 5} \min \left\{\|\eta\|_{\Lambda L^{2}, g_{i}}^{2}, i=1,2\right\} .
$$

The relative error term has the right order, so that any $L^{2}$-norm can be used. Except for the conclusion, any of the two $L^{2}$-norm is simply denoted by \|\| . The comparison of $\mathcal{D}_{g_{1}, f, h}(\omega)$ and $\mathcal{D}_{g_{2}, f, h}(\omega)$ amounts to finding a good estimate for $\left\|\left(d_{f, h}^{*, g_{1}}-d_{f, h}^{*, g_{2}}\right) \omega\right\|$. Let $\omega=\sum_{I} \omega_{I} d x^{I}$ be a $p$-form supported in $\left\{x_{n} \geq-C_{0} h^{2 / 5}\right\}$.

The first point is to observe the inequality

$$
\left\|\left(d_{f, h}^{*, g_{1}}-d_{f, h}^{*, g_{2}}\right) \omega\right\|^{2} \leq C\left(h^{2}\left(\sum_{\ell, I}\left\|\partial_{x_{\ell}}\left(x_{n} \omega_{I}\right)\right\|^{2}\right)+\left\|x_{n}|\nabla f| \omega\right\|^{2}+h^{2}\|\omega\|^{2}\right) .
$$

The second point is to use the Dirichlet realization of $\Delta_{f, h}^{(p)}$, corresponding to Dirichlet boundary conditions on all components. The Weitzenböck formula (actually we only need the structure of the Laplacian and not the detailed intrinsic expression) gives :

$$
\Delta_{f, h}^{(p)}=-h^{2} \sum_{i, j} \nabla_{i} g^{i j}(x) \nabla_{j}+h^{2} R_{(4)}+|\nabla f(x)|^{2}+h\left(\mathcal{L}_{\nabla f}+\mathcal{L}_{\nabla f}^{*}\right) .
$$


The first term is $h^{2}$ times the Bochner Laplacian, while the Ricci curvature $R_{(4)}$ and the term $\left(\mathcal{L}_{\nabla f}+\mathcal{L}_{\nabla f}^{*}\right)$ are tensors with bounded coefficients. We remind that the covariant derivative $\nabla_{i}$ on forms is expressed in terms of the partial derivative $\partial_{x_{j}}$, the Christoffel symbols $\Gamma_{i, \ell}^{j}$ and of the gradient $\nabla x_{m}$ of the coordinate function $x_{m}$ :

$$
\nabla_{i}=\partial_{x_{i}}-\sum_{j, m, \ell} \Gamma_{i, \ell}^{j} g_{j m} d x_{\ell} \wedge\left(\mathbf{i}_{\nabla x_{m}} .\right)
$$

By writing the two sides of (3.9) as quadratic forms on $\Lambda^{p} H_{0}^{1}\left(\mathbb{R}_{-}^{n}\right)$, we get, for any $p$-form $\tilde{\omega}$ such that $\left.\tilde{\omega}\right|_{\left\{x_{n}=0\right\}}=0$, the estimate

$$
\left(h^{2}\left(\sum_{\ell, I}\left\|\partial_{\ell}\left(\tilde{\omega}_{I}\right)\right\|^{2}\right)+\||\nabla f| \tilde{\omega}\|^{2}\right) \leq C\left(\left\|d_{f, h} \tilde{\omega}\right\|^{2}+\left\|d_{f, h}^{*, g .} \tilde{\omega}\right\|^{2}+h\|\tilde{\omega}\|^{2}\right) .
$$

We apply this inequality with $\tilde{\omega}=x_{n} \omega$, which satisfies the full Dirichlet condition. With (3.8), this leads to the inequality :

$$
\left\|\left(d_{f, h}^{*, g_{1}}-d_{f, h}^{*, g_{2}}\right) \omega\right\|^{2} \leq C\left(\left\|d_{f, h} x_{n} \omega\right\|^{2}+\left\|d_{f, h}^{*, g \cdot} x_{n} \omega\right\|^{2}+h\left\|x_{n} \omega\right\|^{2}+h^{2}\|\omega\|^{2}\right) .
$$

It remains to commute $x_{n}$ and $d_{f, h}$ or $d_{f, h}^{*, g}$ and we get, using also our assumption that $\left|x_{n}\right| \leq C h^{\frac{2}{5}}$ in $\operatorname{supp} \omega$,

$$
\left\|\left(d_{f, h}^{*, g_{1}}-d_{f, h}^{*, g_{2}}\right) \omega\right\|^{2} \leq C\left(h^{\frac{4}{5}}\left\|d_{f, h} \omega\right\|^{2}+h^{\frac{4}{5}}\left\|d_{f, h}^{*, g .} \omega\right\|^{2}+h^{\frac{9}{5}}\|\omega\|^{2}\right) .
$$

We conclude with

$$
\begin{aligned}
(1- & \left.C h^{2 / 5}\right)^{-1} \mathcal{D}_{g_{2}, f, h}(\omega)=\left(1-C h^{2 / 5}\right)^{-1}\left[\left\|d_{f, h}^{*, g_{2}} \omega\right\|_{\Lambda L^{2}, g_{2}}^{2}+\left\|d_{f, h} \omega\right\|_{\Lambda L^{2}, g_{2}}^{2}\right] \\
& \geq\left\|d_{f, h}^{*, g_{2}} \omega\right\|_{\Lambda L^{2}, g_{1}}^{2}+\left\|d_{f, h} \omega\right\|_{\Lambda L^{2}, g_{1}}^{2} \\
& \geq\left(\left\|d_{f, h}^{*, g_{1}} \omega\right\|_{\Lambda L^{2}, g_{1}}-\left\|\left(d_{f, h}^{*, g_{2}}-d_{f, h}^{*, g_{1}}\right) \omega\right\|_{\Lambda L^{2}, g_{2}}\right)^{2}+\left\|d_{f, h} \omega\right\|_{\Lambda L^{2}, g_{1}}^{2} \\
& \geq\left(1-h^{2 / 5}\right) \mathcal{D}_{g_{1}, f, h}(\omega)+\left(1-\frac{1}{h^{2 / 5}}\right)\left\|\left(d_{f, h}^{*, g_{2}}-d_{f, h}^{*, g_{1}}\right) \omega\right\|_{\Lambda L^{2}, g_{1}}^{2}
\end{aligned}
$$

and estimate (3.13).

The second result permits to consider again a simpler metric with $g_{n n}=1$. 


\section{Lemma 3.12.}

Let $g_{1}$ and $g_{2}$ be two conformal metrics which satisfy (3.5) and:

$$
g_{2}=e^{\varphi(x)} g_{1} .
$$

Let $f$ be a function satisfying Assumption 3.8. Then there exist constants $C_{12} \geq 1$ and $h_{0}>0$, such that the inequality,

$$
\forall \omega \in \Lambda^{p} H_{0, T}^{1}\left(\mathbb{R}_{-}^{n}\right), \quad \mathcal{D}_{g_{2}, f, h}(\omega) \geq C_{12}^{-1} \mathcal{D}_{g_{1}, f, h}(\omega)-C_{12} h^{2}\|\omega\|_{\Lambda^{p} L^{2}, g_{1}}^{2}
$$

holds, for all $p \in\{0, \ldots, n\}$ and all $h \in\left(0, h_{0}\right)$.

Proof.

For a given metric $g=\sum_{i, j=1}^{n} g_{i j} d x_{i} d x_{j}$, which satisfies property (3.5) and $G=\left(g_{i j}\right)_{1 \leq i, j \leq n}$, the normalized volume form equals

$$
V_{g}(d x)=(\operatorname{det} G(x))^{1 / 2} d x_{1} \wedge \ldots \wedge d x_{n},
$$

the pointwise scalar product of two $p$-forms equals

$$
\langle\omega \mid \eta\rangle_{g}(x)=\sum_{\# I=p} \omega_{I}\left(\Gamma_{p}\left(G^{-1}\right) \bar{\eta}\right)_{I}(x),
$$

with $\Gamma_{p}(A)=A \otimes \cdots \otimes A$, and the Hodge operator is given by

$$
\omega \wedge\left(\star_{g} \bar{\eta}\right)(x)=\langle\omega \mid \eta\rangle_{g}(x) V_{g}(d x)
$$

The term which requires some care in the conformal change of metric in

$$
\mathcal{D}_{g, f, h}(\omega)=\left\|d_{f, h}^{*, g} \omega\right\|_{\Lambda^{p-1} L^{2}, g}^{2}+\left\|d_{f, h} \omega\right\|_{\Lambda^{p+1} L^{2}, g}^{2}
$$

is the first one, because $d_{f, h}^{*, g}$ depends on $g$. We have indeed

$$
d_{f, h}^{*, g} \omega=e^{f / h}(-1)^{p} \star_{g} d \star_{g} e^{-f / h} \omega, \quad \forall \omega \in \Lambda^{p} H^{1} .
$$

Let $g_{1}$ and $g_{2}$ be as above. Our assumptions imply the uniform estimate

$$
\sup _{x \in \overline{\mathbb{R}_{-}^{n}}}|\varphi(x)|<+\infty \text {. }
$$

The previous identities give, for two $p$-forms $\omega$ and $\eta$, the pointwise relations :

$$
\langle\omega \mid \eta\rangle_{g_{2}}(x)=e^{-p \varphi(x)}\langle\omega \mid \eta\rangle_{g_{1}}(x)
$$


and

$$
\begin{aligned}
\omega \wedge\left(\star_{g_{2}} \eta\right) & =\langle\omega \mid \bar{\eta}\rangle_{g_{2}} V_{g_{2}}(d x) \\
& =e^{-p \varphi(x)}\langle\omega \mid \bar{\eta}\rangle_{g_{1}} e^{\frac{n \varphi(x)}{2}} V_{g_{1}}(d x)=e^{\left(-p+\frac{n}{2}\right) \varphi(x)} \omega \wedge\left(\star_{g_{1}} \eta\right)
\end{aligned}
$$

which yields

$$
\star_{g_{2}} \eta=e^{\left(-p+\frac{n}{2}\right) \varphi(x)} \star_{g_{1}} \eta .
$$

Let us compute first with $f=0$ and $h=1$, the pointwise scalar product

$$
\begin{aligned}
& \left\langle d^{*, g_{2}} \omega \mid d^{*, g_{2}} \eta\right\rangle_{g_{2}}(x)=e^{-(p-1) \varphi(x)}\left\langle d^{*, g_{2}} \omega \mid d^{*, g_{2}} \eta\right\rangle_{g_{1}}(x) \\
& \quad=e^{-(p-1) \varphi(x)}\left\langle\star_{g_{2}} d \star_{g_{2}} \omega \mid \star_{g_{2}} d \star_{g_{2}} \eta\right\rangle_{g_{1}}(x) \\
& \quad=e^{-(p-1) \varphi(x)}\left\langle\star_{g_{2}} d \star_{g_{1}}\left(e^{(p+n / 2) \varphi(x)} \omega\right) \mid \star_{g_{2}} d \star_{g_{1}}\left(e^{(p+n / 2) \varphi(x)} \eta\right)\right\rangle_{g_{1}}(x) \\
& =e^{-(p-1) \varphi(x)} e^{2(p-1-n / 2) \varphi(x)}\left\langle d^{*, g_{1}}\left(e^{(p+n / 2) \varphi(x)} \omega\right) \mid d^{*, g_{1}}\left(e^{(p+n / 2) \varphi(x)} \eta\right)\right\rangle_{g_{1}}(x) .
\end{aligned}
$$

Hence we get

$$
\begin{aligned}
& \left\langle d^{*, g_{2}} \omega \mid d^{*, g_{2}} \eta\right\rangle_{g_{2}}(x) V_{g_{2}}(d x) \\
& \quad=e^{(p-1) \varphi(x)}\left\langle d^{*, g_{1}}\left(e^{(p+n / 2) \varphi(x)} \omega\right) \mid d^{*, g_{1}}\left(e^{(p+n / 2) \varphi(x)} \eta\right)\right\rangle_{g_{1}}(x) V_{g_{1}}(d x) .
\end{aligned}
$$

and

$$
\left\|d^{*, g_{2}} \omega\right\|_{\Lambda L^{2}, g_{2}}^{2} \geq e^{-(p-1) \mu}\left\|d^{*, g_{1}} \omega\right\|_{\Lambda L^{2}, g_{1}}^{2} .
$$

With $f$ and $h \in\left(0, h_{0}\right)$, this gives the existence of $C>0$ such that :

$$
\begin{aligned}
\left\|e^{f / h} h d^{*, g_{2}}\left(e^{-f / h} \omega\right)\right\|_{\Lambda L^{2}, g_{2}}^{2} & \geq e^{-(p-1) \mu}\left\|e^{f / h} h d^{*, g_{1}}\left(e^{-f / h+(p+n / 2) \varphi} \omega\right)\right\|_{\Lambda L^{2}, g_{1}}^{2} \\
& \geq e^{-(3 p-1+n) \mu}\left\|\left(d_{f, h}^{*, g_{1}}+h \mathbf{i}_{\nabla(p+n / 2) \varphi}\right) \omega\right\|_{\Lambda L^{2}, g_{1}}^{2} \\
& \geq C^{-1}\left\|d_{f, h}^{*, g_{1}} \omega\right\|_{\Lambda L^{2}, g_{1}}^{2}-C h^{2}\|\omega\|_{\Lambda L^{2}, g_{1}}^{2} .
\end{aligned}
$$

\section{Proposition 3.13.}

Assume that the metric $g$ satisfies (3.4) and (3.5) and let $f$ be a Morse function satisfying Assumption 3.8. Then there exist constants $h_{0}>0, c_{0}>0$ and $c_{1}>0$, such that the self-adjoint operator $\Delta_{f, h}^{D T}$ satisfies the following properties for $h \in\left(0, h_{0}\right]$ :

i) The essential spectrum $\sigma_{\text {ess }}\left(\Delta_{f, h}^{D T,(p)}\right)$ is contained in $\left[c_{1},+\infty\right)$. 
ii) For $p \in\{0, \ldots, n\}$, the range of the spectral projection $1_{\left[0, c_{0} h\right)}\left(\Delta_{f, h}^{D T,(p)}\right)$ has dimension

$$
\left\{\begin{array}{lll}
\delta_{p, p_{0}+1} & \text { if } & \partial_{x_{n}} f(0)=-\frac{1}{2} \partial_{x_{n}} f_{+}(0)>0 \\
0 & \text { if } & \partial_{x_{n}} f(0)=-\frac{1}{2} \partial_{x_{n}} f_{+}(0)<0
\end{array} .\right.
$$

iii) In the case when $\partial_{x_{n}} f(0)=-\frac{1}{2} \partial_{x_{n}} f_{+}(0)>0$, the spectral subspace associated with the small eigenvalues of $\Delta_{f, h}^{D T,\left(p_{0}+1\right)}$ equals :

$$
\operatorname{Ran} 1_{\left[0, c_{0} h\right)}\left(\Delta_{f, h}^{D T,\left(p_{0}+1\right)}\right)=\operatorname{Ker} \Delta_{f, h}^{D T,\left(p_{0}+1\right)}=\mathbb{C} \varphi^{h},
$$

where

$$
\left\|\varphi^{h}-\psi_{p_{0}}^{h} \wedge\left(e^{-f_{+}\left(x_{n}\right) / 2 h} d x_{n}\right)\right\|_{\Lambda^{p} L^{2}}=\mathcal{O}\left(h^{1 / 10}\right),
$$

and $\psi_{p_{0}}^{h}$ belongs to the kernel of an $(n-1)$-dimensional Witten Laplacian $\Delta_{\tilde{g}^{\prime}, f_{-} / 2, h}^{\left(p_{0}\right)}$ in a metric $\tilde{g}^{\prime}$, which is conformal to $g^{\prime}=\sum_{i, j=1}^{n-1} g_{i j}\left(x^{\prime}, 0\right) d x_{i} d x_{j}$ on $\mathbb{R}^{n-1}$.

iv) For any $\chi \in \mathcal{C}_{0}^{\infty}\left(\overline{\mathbb{R}_{-}^{n}}\right)$ such that $\chi=1$ in a neighborhood of 0 , there exists $C_{\chi}>0$ such that the lower bounds

$$
(1-\chi) \Delta_{f, h}^{D T,(p)}(1-\chi) \geq C_{\chi}^{-1}[1-\chi]^{2}, 0 \leq p \leq n
$$

hold, for any $h \in\left(0, h_{0} / C_{\chi}\right)$, in the sense of quadratic form on $\Lambda^{p} H_{0, T}^{1}\left(\mathbb{R}_{-}^{n}\right)$.

Proof.

The clue of this result is an accurate lower bound for the quadratic form $\mathcal{D}_{g, f, h}(\eta)$, when evaluated for $\eta$ such that $\operatorname{supp} \eta \subset\left\{x_{n} \geq-C_{0} h^{2 / 5}\right\}$. By Lemmas 3.11 and 3.12, one can find a metric $\tilde{g}$, which satisfies (3.4) and (3.5), with $\tilde{G}(x)=\tilde{G}\left(x^{\prime}\right)$ independent of the $x_{n}$-coordinate, $\tilde{g}_{n n}=1$ and a constant $C>1$ such that

$$
\mathcal{D}_{g, f, h}(\eta) \geq C^{-1} \mathcal{D}_{\tilde{g}, f, h}(\eta)-C h^{7 / 5}\|\eta\|_{\Lambda L^{2}, \tilde{g}}^{2}
$$

Take two cut-off functions $\tilde{\chi}_{i} \in \mathcal{C}^{\infty}(\mathbb{R})$, such that $\tilde{\chi}_{1} \in \mathcal{C}_{0}^{\infty}(\mathbb{R}), \tilde{\chi}_{1}=1$ in a neighborhood of 0 such that $\tilde{\chi}_{1}^{2}+\tilde{\chi}_{2}^{2}=1$. This partition of unity gives, for any $\omega \in \Lambda H_{0, T}^{1}\left(\mathbb{R}_{-}^{n}\right)$,

$$
\mathcal{D}_{g, f, h}(\omega) \geq \mathcal{D}_{g, f, h}\left(\tilde{\chi}_{1}\left(h^{-2 / 5} x_{n}\right) \omega\right)+\mathcal{D}_{g, f, h}\left(\tilde{\chi}_{2}\left(h^{-2 / 5} x_{n}\right) \omega\right)-C h^{6 / 5}\|\omega\|_{\Lambda L^{2}, g}^{2}
$$


Since $|\nabla f(x)|^{2} \geq C^{-1}$ on $\mathbb{R}_{-}^{n}$, the second term is bounded from below by $\left.\| \tilde{\chi}_{2}\left(h^{-2 / 5} x_{n}\right)\right) \omega \|_{\Lambda L^{2}, g}^{2}$. Hence we get

$$
\begin{aligned}
\mathcal{D}_{g, f, h}(\omega) \geq \mathcal{D}_{g, f, h}\left(\tilde{\chi}_{1}\left(h^{-2 / 5} x_{n}\right) \omega\right)-C h^{6 / 5} & \left\|\tilde{\chi}_{1}\left(h^{-2 / 5} x_{n}\right) \omega\right\|_{\Lambda L^{2}, g}^{2} \\
& +\frac{C^{-1}}{2}\left\|\tilde{\chi}_{2}\left(h^{-2 / 5} x_{n}\right) \omega\right\|_{\Lambda L^{2}, g}^{2} .
\end{aligned}
$$

Finally after changing the constant $C \geq 1$, the inequality (3.15) yields

$$
\begin{aligned}
\mathcal{D}_{g, f, h}(\omega) \geq C^{-1} \mathcal{D}_{\tilde{g}, f, h}\left(\tilde{\chi}_{1}\left(h^{-2 / 5} x_{n}\right) \omega\right)-C h^{6 / 5}\left\|\tilde{\chi}_{1}\left(h^{-2 / 5} x_{n}\right) \omega\right\|^{2} \\
+C^{-1}\left\|\tilde{\chi}_{2}\left(h^{-2 / 5} x_{n}\right) \omega\right\|^{2}
\end{aligned}
$$

where the $L^{2}$-norms in the right hand side can be computed with the metric $g$ or $\tilde{g}$ while possibly adapting the constant $C$. Here and in the sequel, we omit the subscript $\left(\Lambda L^{2}, g\right)$ for $L^{2}$-norms.

Now the problem is reduced to the analysis of $\mathcal{D}_{\tilde{g}, f, h}$ with the metric $\tilde{g}$.

(a) The case $n=1$.

We have $x=x_{n} \in \mathbb{R}_{-}, f(x)=-\frac{1}{2} f_{+}\left(x_{n}\right)$. Here the metric is $\tilde{g}=d x_{n}^{2}$. We keep the reference to the index $n$ for the later application.

The space $\Lambda^{0} H_{0, T}^{1}\left(\mathbb{R}_{-}\right)$is simply $H_{0}^{1}\left(\mathbb{R}_{-}\right)$, while

$$
\Lambda^{1} H_{0, T}^{1}=\left\{\alpha\left(x_{n}\right) d x_{n}, \alpha \in H^{1}\left(\mathbb{R}_{-}\right)\right\} .
$$

The identity (2.15) reads :

$\forall \beta \in H_{0}^{1}\left(\mathbb{R}_{-}\right), \quad \mathcal{D}_{\tilde{g},-f_{+} / 2, h}(\beta)=h^{2}\left\|\partial_{x_{n}} \beta\right\|^{2}+\frac{h^{2}}{4}\left\|\partial_{x_{n}} f_{+} \beta\right\|^{2}+\frac{h}{2}\left\langle\partial_{x_{n}}^{2} f_{+}\left(x_{n}\right) \beta \mid \beta\right\rangle$,

for the 0 -forms and for the 1-forms :

$$
\begin{aligned}
\forall \alpha \in H^{1}\left(\mathbb{R}_{-}\right), \quad \mathcal{D}_{\tilde{g},-f_{+} / 2, h}(\alpha & \left.d x_{n}\right)=h^{2}\left\|\partial_{x_{n}} \alpha\right\|^{2}+\frac{h^{2}}{4}\left\|\partial_{x_{n}} f_{+} \alpha\right\|^{2} \\
& +h\left\langle\partial_{x_{n}}^{2} f_{+}\left(x_{n}\right) \alpha \mid \alpha\right\rangle+\frac{h}{2} \partial_{x_{n}} f_{+}(0)|\alpha(0)|^{2} .
\end{aligned}
$$

On 0-forms, we get

$$
\forall \beta \in \Lambda^{0} H_{0, T}^{1}, \quad \mathcal{D}_{\tilde{g},-f_{+} / 2, h}(\beta) \geq\left(C^{-2}-h C\right)\|\beta\|^{2},
$$


and deduce that there exist $c_{1}>0$ and $h_{0}>0$ such that, for all $h \in\left(0, h_{0}\right]$,

$$
\Delta_{\tilde{g},-f_{+} / 2, h}^{D T,(0)} \geq c_{1} \mathrm{Id} .
$$

On 1-forms, there are two subcases :

(a1) Subcase $\partial_{x_{n}} f_{+}(0)>0$ :

The inequality,

$$
\forall \alpha \in H_{0, T}^{1}, \quad \mathcal{D}_{\tilde{g},-f_{+} / 2, h}\left(\alpha d x_{n}\right) \geq\left(C^{-2}-h C\right)\|\alpha\|^{2}
$$

implies the existence of $h_{0}>0$ such that

$$
\Delta_{-f_{+} / 2, h}^{D T,(1)} \geq c_{1} \mathrm{Id}, \forall h \in\left(0, h_{0}\right] \text {. }
$$

(a2) Subcase $\partial_{\mathbf{x}_{\mathbf{n}}} f_{+}(0)<0$ :

If $\Delta_{-f_{+} / 2, h}^{D T,(1)}\left(\alpha d x_{n}\right)=\lambda^{h} \alpha d x_{n}$, with $\lambda_{h}<c_{1}$, Proposition 3.9 implies

$$
d_{-f_{+} / 2, h}^{*}\left(\alpha d x_{n}\right)=0
$$

which means

$$
\alpha\left(x_{n}\right)=C e^{-f_{+}\left(x_{n}\right) / 2 h} .
$$

The 1 -form $e^{-f_{+}\left(x_{n}\right) / 2 h} d x_{n}$ belongs to $\operatorname{Ker}\left(\Delta_{-f_{+} / 2, h}^{D T,(1)}\right)$.

(b) The case $n>1$.

First note that any $\omega \in \Lambda^{p} H_{0, T}^{1}\left(\mathbb{R}_{-}^{n}\right)$ is a sum

$$
\omega=\sum_{\# I=p-1} \alpha_{I}(x) d x^{\prime I} \wedge d x_{n}+\sum_{\# J=p} \beta_{J}(x) d x^{\prime J}=: \alpha \wedge d x_{n}+\beta,
$$

with $\alpha_{I}, \beta_{J} \in H^{1}\left(\mathbb{R}_{-}^{n}\right), \beta_{J}\left(x^{\prime}, 0\right)=0$, while $d x^{\prime I}=d x_{i_{1}}^{\prime} \wedge \cdots \wedge d x_{i_{\# I}}^{\prime}$, $I=\left\{i_{1}<\ldots<i_{\# I}\right\} \subset\{1, \ldots, n-1\}$.

If in addition $\omega \in \Lambda^{p} H^{2}\left(\mathbb{R}_{-}^{n}\right)$, the condition $\mathbf{t} d^{*} \omega=0$ reads $\partial_{x_{n}} \alpha\left(x^{\prime}, 0\right)=0$ (for the metric $\tilde{g}$ ). Hence the variables $\left(x^{\prime}, x_{n}\right)$ can be separated and $\mathcal{D}_{\tilde{g}, f, h}(\omega)$ equals

$$
\begin{aligned}
& \int_{\mathbb{R}^{n-1}}\left[\sum_{\# I=p-1} \mathcal{D}_{-f_{+} / 2, h}^{n}\left(\alpha_{I}\left(x^{\prime}, .\right) d x_{n}\right)+\sum_{\# J=p} \mathcal{D}_{-f_{+} / 2, h}^{n}\left(\beta_{J}\left(x^{\prime}, .\right)\right)\right] d \lambda\left(x^{\prime}\right) \\
& +\int_{-\infty}^{0} \mathcal{D}_{f_{-} / 2, h}^{\prime}\left(\alpha\left(., x_{n}\right)\right)+\mathcal{D}_{f_{-} / 2, h}^{\prime}\left(\beta\left(., x_{n}\right)\right) d x_{n},
\end{aligned}
$$


where we used the notations $\mathcal{D}_{f_{-} / 2, h}^{\prime}$ for the quadratic form of the Witten Laplacian on $\mathbb{R}^{n-1}$ and $\mathcal{D}_{-f_{+} / 2, h}^{n}$ for the quadratic form of the 1-dimensional Witten Laplacian on $\mathbb{R}_{-}$with boundary conditions. The measure $d \lambda\left(x^{\prime}\right)$ simply equals $\left(\operatorname{det} \tilde{G}\left(x^{\prime}\right)\right)^{1 / 2} d x^{\prime}$.

Again there are two subcases.

(b1) Subcase $\partial_{x_{n}} f_{+}(0)>0$ :

The analysis of the one dimensional problem implies

and

$$
\mathcal{D}_{-f_{+} / 2, h}^{n}\left(\alpha_{I}\left(x^{\prime}, .\right) d x_{n}\right) \geq c_{1}\left\|\alpha_{I}\left(x^{\prime}, .\right)\right\|^{2}
$$

$$
\mathcal{D}_{-f_{+} / 2, h}^{n}\left(\beta_{J}\left(x^{\prime}, .\right)\right) \geq c_{1}\left\|\beta_{J}\left(x^{\prime}, .\right)\right\|^{2} .
$$

Hence we get

$$
\forall \omega \in \Lambda^{p} H_{0, T}^{1}, \quad \mathcal{D}_{\tilde{g}, f, h}(\omega) \geq c_{1}\|\omega\|^{2}
$$

and there exists $c_{1}>0$ such that

$$
\Delta_{f, h}^{D T,(p)} \geq c_{1} \mathrm{Id}, \forall p \in\{0, \ldots, n\} .
$$

(b2) Subcase $\partial_{x_{n}} f_{+}(0)<0$ :

Then there exists $c_{1}>0$ such that

$$
\begin{aligned}
\mathcal{D}_{\tilde{g}, f, h}(\omega) \geq \int_{\mathbb{R}^{n-1}} \sum_{\# I=p-1} \mathcal{D}_{-f_{+} / 2, h}^{n}\left(\alpha_{I}\left(x^{\prime}, .\right) d x_{n}\right) d \lambda\left(x^{\prime}\right) \\
\quad+\int_{-\infty}^{0} \mathcal{D}_{f_{-} / 2, h}^{\prime}\left(\alpha\left(., x_{n}\right)\right) d x_{n}+c_{1}\|\beta\|^{2} .
\end{aligned}
$$

If $\omega$ is a $p$-form with $p \neq p_{0}+1$, the lower bound

$$
\mathcal{D}_{f_{-} / 2, h}^{\prime}(\alpha) \geq C_{1}^{-1} h\|\alpha\|^{2},
$$

which was given in Proposition 3.7, yields :

$$
\mathcal{D}_{\tilde{g}, f, h}(\omega) \geq C^{-1} h\|\omega\|^{2},
$$

while the equality $\mathcal{D}_{\tilde{g}, f, h}(\omega)=0$ implies that $p=p_{0}+1$ and that $\omega=c \psi_{p_{0}}^{h} \wedge\left(e^{-f_{+}\left(x_{n}\right) / 2 h} d x_{n}\right)$, where $\psi_{p_{0}}$ belongs to the kernel of the $(n-1)$ dimensional Witten Laplacian associated with the metric

$$
\tilde{g^{\prime}}=\sum_{i, j=1}^{n-1} \tilde{g}_{i, j}\left(x^{\prime}, 0\right) d x_{i} d x_{i} \text {. }
$$


We have now all the ingredients to check every statement for a general metric $g$. We focus on the subcase $\partial_{x_{n}} f_{+}(0)<0$, which covers all possibilities.

\section{Statements i) and iv)}

Statement i) is a consequence of iv) together with Persson's Lemma. It is sufficient to check that, for all $R>0$, there exists $c_{R}>0$, such that, for all $\omega \in \Lambda^{p} H_{0, T}^{1}\left(\mathbb{R}^{n_{-}}\right)$supported in $\left\{\min \left(\left|x^{\prime}\right|,\left|x_{n}\right|\right)>R\right\}$, one has

$$
\mathcal{D}_{g, f, h}(\omega) \geq c_{R}\|\omega\|^{2}
$$

The inequalities (3.16) and (3.17), together with the estimate

$$
\mathcal{D}_{f_{-} / 2, h}^{\prime}\left(\alpha_{I}\left(\cdot, x_{n}\right)\right) \geq c_{R}^{\prime}\left\|\alpha\left(\cdot, x_{n}\right)\right\|^{2} \quad \text { if } \operatorname{supp} \omega \subset\left\{\left|x^{\prime}\right|>R\right\},
$$

provided by Proposition 3.7-iii), yield the result.

Statements ii) and iii)

If $p \neq p_{0}+1$ the inequalities (3.16), (3.17) and the inequality,

$$
\mathcal{D}_{f_{-} / 2, h}^{\prime}\left(\alpha\left(., x_{n}\right)\right) \geq C^{-1} h\left\|\alpha\left(., x_{n}\right)\right\|^{2},
$$

imply

$$
\mathcal{D}_{g, f, h}(\omega) \geq c_{0} h\|\omega\|^{2}
$$

and

$$
\Delta_{f, h}^{D T,(p)} \geq c_{0} h \mathrm{Id}
$$

By Proposition 3.9, the only possibility, for $\lambda_{h} \in\left[0, c_{0} h\right)$, to be an eigenvalue of $\Delta_{f, h}^{D T,\left(p_{0}+1\right)}$ is $\lambda_{h}=0$. When $g=\tilde{g}$ the corresponding spectral subspace is one dimensional and equals $\mathbb{C} \psi_{p_{0}} \wedge\left(e^{-f_{+}\left(x_{n}\right) / 2 h} d x_{n}\right)$. For a general metric $g$, the equation $\Delta_{g, f, h}^{D T,\left(p_{0}+1\right)} \omega=0,\|\omega\|=1$, which implies $\mathcal{D}_{g, f, h}(\omega)=0$, and the inequality (3.16) leads to

$$
C^{2} h^{6 / 5}\left\|\tilde{\chi}_{1}\left(h^{-2 / 5} x_{n}\right) \omega\right\|^{2} \geq \mathcal{D}_{\tilde{g}, f, h}\left(\tilde{\chi}_{1}\left(h^{-2 / 5} x_{n}\right) \omega\right)+\left\|\tilde{\chi}_{2}\left(h^{-2 / 5} x_{n}\right) \omega\right\|^{2} .
$$

Without the last term, Lemma 2.8 implies

$$
\text { dist }{ }_{L^{2}}\left(\tilde{\chi}\left(h^{-2 / 5} x_{n}\right) \omega, \mathbb{C} \psi_{p_{0}} \wedge\left(e^{-f_{+}\left(x_{n}\right) / 2 h} d x_{n}\right)\right) \leq C h^{1 / 10} \text {. }
$$

The upper bound of the last term,

$$
\left\|\tilde{\chi}_{2}\left(h^{-2 / 5} x_{n}\right) \omega\right\|^{2} \leq C h^{6 / 5}
$$


implies

$$
\operatorname{dist} L^{2}\left(\omega, \mathbb{C} \psi_{p_{0}} \wedge\left(e^{-f_{+}\left(x_{n}\right) / 2 h} d x_{n}\right)\right)=\mathcal{O}\left(h^{1 / 10}\right) .
$$

It remains to check that $\operatorname{Ker} \Delta_{f, h}^{D T,\left(p_{0}+1\right)}$ is not reduced to $\{0\}$. The statements of Lemma 3.11 and Lemma 3.12 are symmetric with respect to the choice of the metric. Hence the reverse inequality of (3.16) (with exchange of $g$ and $\tilde{g}$ ),

$$
\begin{aligned}
\mathcal{D}_{\tilde{g}, f, h}(\omega) \geq C^{-1} \mathcal{D}_{g, f, h}\left(\tilde{\chi}_{1}\left(h^{-2 / 5} x_{n}\right) \omega\right)-C h^{6 / 5}\left\|\tilde{\chi}_{1}\left(h^{-2 / 5} x_{n}\right) \omega\right\|^{2} \\
+C^{-1}\left\|\tilde{\chi}_{2}\left(h^{-2 / 5} x_{n}\right) \omega\right\|^{2},
\end{aligned}
$$

also holds for any $\omega \in \Lambda H_{0, T}^{1}\left(\mathbb{R}_{-}^{n}\right)$. We apply it with $\left.\omega=\psi_{p_{0}} \wedge\left(e^{-f_{+}\left(x_{n}\right) / 2 h} d x_{n}\right)\right)$ and this leads to

$$
\mathcal{D}_{g, f, h}\left(\tilde{\chi}\left(h^{-2 / 5} x_{n}\right) \omega^{h}\right) \leq C h^{6 / 5} .
$$

The Min-Max principle then says that $\Delta_{f, h}^{D T,\left(p_{0}+1\right)}$ admits an eigenvalue smaller than $C h^{6 / 5}$. It has to be 0 due to the previous supersymmetric argument.

\subsection{Reduction to the local half-space problem.}

We end here the proof of Theorem 3.3 by introducing, after a partition of unity, the right coordinates which permit the comparison with the model half-space problem.

Proof of Theorem 3.3.

Let $\left\{U_{k}, 1 \leq k \leq K\right\}$ denote the union of the critical points of $f$ and $\left.f\right|_{\Omega}$. Consider a partition of unity $\sum_{k=1}^{N} \chi_{k}^{2}=1$ such that the $\mathcal{C}_{0}^{\infty}(\bar{\Omega})$ function $\chi_{k}$ identically equals 1 in a neighborhood of $U_{k}$ when $1 \leq k \leq K$. The refinement of this partition of unity will be specified later by the local construction of adapted coordinates.

We recall that the operator $\Delta_{f, h}^{D T}$ is the Friedrichs extension associated with the quadratic form :

$$
\mathcal{D}_{g, f, h}(\omega)=\left\|d_{f, h} \omega\right\|_{\Lambda L^{2}, g}^{2}+\left\|d_{f, h}^{*, g} \omega\right\|_{\Lambda L^{2}, g}^{2}
$$

on $\Lambda H_{0, T}^{1}(\Omega)$. The standard IMS localization formula $([\mathrm{CFKS}])$ gives

$$
\mathcal{D}_{g, f, h}(\omega)=\sum_{k=1}^{N} \mathcal{D}_{g, f, h}\left(\chi_{k} \omega\right)-h^{2}\left\|\left|\nabla \chi_{k}\right| \omega\right\|_{\Lambda L^{2}, g}^{2}
$$


for any $\omega \in D\left(\Delta_{f, h}^{D T}\right)$ and by density for any $\omega \in \Lambda H_{0, T}^{1}$.

If $\operatorname{supp} \chi_{k}$ does not meet the boundary, the term $\mathcal{D}_{g, f, h}\left(\chi_{k} \omega\right)$ behaves like in the boundaryless case :

- If $k>K$, then we have

$$
\mathcal{D}_{g, f, h}\left(\chi_{k} \omega\right) \geq C^{-1}\|\omega\|_{\Lambda L^{2}, g}^{2} .
$$

- If $k \leq K, \omega \in \Lambda^{p} H_{0, T}^{1}$ and $U_{k}$ is a critical point of $f$ with index $p_{k} \neq p$, then $\mathcal{D}_{g, f, h}\left(\chi_{k} \omega\right) \geq C^{-1} h$.

- If $k \leq K, \omega \in \Lambda^{p} H_{0, T}^{1}$ and $U_{k}$ is a critical point of $f$ with index $p_{k}=p$, then there exists a fixed 1-dimensional space $F_{k}^{(p)}$ determined by Hess $f\left(U_{k}\right)$ such that,

$$
\mathcal{D}_{g, f, h}\left(\chi_{k} \omega\right) \leq C^{-1} h^{6 / 5}\|\omega\|_{\Lambda^{p} L^{2}, g}^{2}
$$

implies

$$
\operatorname{dist}\left(\chi_{k} \omega, F_{k}^{(p)}\right) \leq C h^{1 / 10}\|\omega\|_{\Lambda^{p} L^{2}, g} .
$$

Again like in the proof of Proposition 3.13-iii), this last statement refers to Lemma 2.8 at the level of quadratic forms.

Consider now the case when $\operatorname{supp} \chi_{k} \cap \partial \Omega \neq \emptyset$, with the support of $\chi_{k}$ centered around a point $U_{0} \in \partial \Omega$. There are two subcases :

1) $\left(\frac{\partial f}{\partial n}\right)\left(U_{0}\right)<\left|\nabla f\left(U_{0}\right)\right|$.

Then the cut-off $\chi_{k}$ is chosen so that, in a neighborhood $\mathcal{V}$ of $\operatorname{supp} \chi_{k}$,

$$
\forall x \in \mathcal{V} \cap \partial \Omega, \quad\left(\frac{\partial f}{\partial n}\right)(x)<(1-\delta)|\nabla f(x)|,
$$

with $\delta>0$. Locally it is possible to construct a function $\hat{f}$ such that $\partial_{n} \hat{f}=|\nabla \hat{f}|$ in $\mathcal{V} \cap \partial \Omega$ and $|\nabla \hat{f}|=|\nabla f|$ in $\mathcal{V}$. By setting $\tilde{\omega}=\chi_{k} \omega$, the Green formula $(2.15)$ and the inequality $\mathcal{D}_{g, \hat{f}, h}(\tilde{\omega}) \geq 0$ imply

$$
\begin{aligned}
& h \int_{\partial \Omega}\langle\tilde{\omega} \mid \tilde{\omega}\rangle_{\Lambda^{p} T_{\sigma}^{*} \Omega}\left(\frac{\partial f}{\partial n}\right)(\sigma) d \sigma \leq(1-\delta) h \int_{\partial \Omega}\langle\tilde{\omega} \mid \tilde{\omega}\rangle_{\Lambda^{p} T_{\sigma}^{*} \Omega}\left(\frac{\partial \hat{f}}{\partial n}\right)(\sigma) d \sigma \\
& \leq(1-\delta)\left[h^{2}\|d \tilde{\omega}\|_{\Lambda^{p+1} L^{2}}^{2}+h^{2}\left\|d^{*} \tilde{\omega}\right\|_{\Lambda^{p-1} L^{2}}^{2}+\||\nabla f| \tilde{\omega}\|_{\Lambda^{p} L^{2}}^{2}+C_{1} h\|\tilde{\omega}\|_{\Lambda^{p} L^{2}}^{2}\right] .
\end{aligned}
$$


With (2.15), we obtain

$$
\mathcal{D}_{g, f, h}\left(\chi_{k} \omega\right)=\mathcal{D}_{g, f, h}(\tilde{\omega}) \geq \frac{\delta}{2}\||\nabla f| \tilde{\omega}\|_{\Lambda^{p} L^{2}}^{2} \geq C_{\mathcal{V}}^{-1}\left\|\chi_{k} \omega\right\|_{\Lambda^{p} L^{2}}^{2}
$$

2) $\partial_{n} f\left(U_{0}\right)=\left|\nabla f\left(U_{0}\right)\right|$.

Then $U_{0} \in \partial \Omega$ is a critical point of $\left.f\right|_{\partial \Omega}$ with $\partial_{n} f>0$. Around $U_{0}$, we now introduce adapted local coordinates. Due to the condition $\partial_{n} f\left(U_{0}\right) \neq 0$, the eikonal equation :

$$
\left|\partial_{n} \Phi\right|^{2}+\left|\nabla_{T} \Phi\right|^{2}=|\nabla \Phi|^{2}=|\nabla f|^{2}
$$

with the boundary condition

$$
\left.\Phi\right|_{\partial \Omega, U_{0}}=\left.f\right|_{\partial \Omega, U_{0}}
$$

admits a second local solution which satisfies

$$
\left.\partial_{n} \Phi\right|_{\partial \Omega, U_{0}}=-\left.\partial_{n} f\right|_{\partial \Omega, U_{0}}
$$

Like in [HelSj4], we set

$$
f_{+}=\Phi-f \quad \text { and } \quad f_{-}=\Phi+f .
$$

We have the relations

$$
\begin{array}{ll} 
& f=-\frac{1}{2} f_{+}+\frac{1}{2} f_{-}, \quad \Phi=\frac{1}{2} f_{+}+\frac{1}{2} f_{-}, \\
& \nabla f_{+} \cdot \nabla f_{-}=0, \\
& \left.f_{+}\right|_{\partial \Omega, U_{0}}=0, \quad-\left.\partial_{n} f_{+}\right|_{\partial \Omega, U_{0}}=\left.2 \partial_{n} f\right|_{\partial \Omega, U_{0}} \neq 0, \\
\text { and } \quad & \left.f_{-}\right|_{\partial \Omega, U_{0}}=\left.2 f\right|_{\partial \Omega, U_{0}},\left.\quad \partial_{n} f_{-}\right|_{\partial \Omega, U_{0}}=0 .
\end{array}
$$

Let $\left(x_{1}, \ldots, x_{n-1}\right)=x^{\prime}$ denote a set of coordinates on $\partial \Omega$ in a neighborhood of $U_{0}$ and such that $x_{j}\left(U_{0}\right)=0$. We extend them in a neighborhood of $U_{0}$ in $\bar{\Omega}$ as constant along the integral curve of the vector field $\nabla f_{+}$. Then we take $x_{n}=-\frac{1}{2} f_{+}(x)$ for the last coordinate. In these coordinates the function $f$ and the metric $g$ have the form

$$
f(x)=x_{n}+\frac{1}{2} f_{-}\left(x^{\prime}\right) \quad \text { and } \quad g=g_{n n}(x) d x_{n}^{2}+\sum_{i, j=1}^{n-1} g_{i j}(x) d x_{i} d x_{j} .
$$


The boundary $\partial \Omega$ is locally defined by $\left\{x_{n}=0\right\}$ and $\Omega$ corresponds to $\left\{x_{n}<0\right\}$. In order to apply Proposition 3.13, it remains to check that the function $f$ can be extended to $\mathbb{R}_{-}^{n}$, so that it satisfies Assumption 3.8 when $U_{0}$ is a critical point $\left.f\right|_{\partial \Omega}$. Indeed the additional assumption does not depend on the metric $g$ and we can assume that the $g_{i j}$ are constant in the coordinates $\left(x^{\prime}, x_{n}\right)$. It suffices to choose, in a small enough neighborhood of $U_{0}=(0, \ldots, 0)$, Morse coordinates $\left(x_{1}, \ldots, x_{n}\right)$ for $f_{-}$in the metric $\sum_{i, j<n} g_{i j} d x_{i} d x_{j}$ :

$$
f(x)=x_{n}+\sum_{j=1}^{n-1} \lambda_{j} x_{j}^{2}
$$

Then this function is extended to $\mathbb{R}_{-}^{n}$ by :

$$
\tilde{f}(x)=x_{n}+\left[\chi\left(x^{\prime}\right)+\frac{1-\chi\left(x^{\prime}\right)}{\left|x^{\prime}\right|}\right]\left[\sum_{j=1}^{n-1} \lambda_{j} x_{j}^{2}\right],
$$

for some cut-off function $\chi$ supported in a neighborhood of $x^{\prime}=0$.

With this choice of coordinates, the quantity $\mathcal{D}_{g, f, h}\left(\chi_{k} \omega\right)$ take the form discussed in Proposition 3.13.

We can now discuss the lower bound of $\mathcal{D}_{g, f, h}\left(\chi_{k} \omega\right)$, depending on the localization by the cut-off $\chi_{k}$, such that $\operatorname{supp} \chi_{k} \cap \partial \Omega \neq \emptyset$.

- If $k>K$, we are in case 1) and

$$
\mathcal{D}_{g, f, h}\left(\chi_{k} \omega\right) \geq C^{-1}\left\|\chi_{k} \omega\right\|_{\Lambda L^{2}, g}^{2} .
$$

- If $k \leq K$, the origin of the coordinate system is $U_{0}=U_{k}$. If $\omega \in$ $\Lambda^{p} H_{0, T}^{1}$ and $U_{k}$ is not a critical point of $\left.f\right|_{\partial \Omega}$ with index $p_{k}=p-1$ and $\partial_{n} f\left(U_{k}\right)>0$, then $\mathcal{D}_{g, f, h}\left(\chi_{k} \omega\right) \geq C^{-1} h$.

- If $k \leq K, \omega \in \Lambda^{p} H_{0, T}^{1}$ and $U_{k}$ is a critical point of $\left.f\right|_{\partial \Omega}$ with index $p_{k}=p-1$ and $\partial_{n} f\left(U_{k}\right)>0$, then according to Proposition 3.13-iii) there exists a fixed 1-dimensional space $F_{k}^{(p)}$ such that the inequality,

$$
\mathcal{D}_{g, f, h}\left(\chi_{k} \omega\right) \leq C^{-1} h^{6 / 5}\left\|\chi_{k} \omega\right\|_{\Lambda^{p} L^{2}, g}^{2},
$$

implies :

$$
\operatorname{dist}\left(\chi_{k} \omega, F_{k}^{(p)}\right) \leq C h^{1 / 10}\|\omega\|_{\Lambda^{p} L^{2}, g}
$$


The partition of unity is chosen so that the previous choice of coordinates is possible in a neighborhood of any $\chi_{k}$ and the set of metrics $g=g_{n n}(x) d x_{n}{ }^{2}+\sum_{i, j<n} g_{i j} d x_{i} d x_{j}$ in the local coordinate systems form a $\mathcal{K}$-set according to Definition 3.10. Hence, the constant $C$ can be chosen uniformly. We now introduce the set $A_{p}$ of indices $k, 1 \leq k \leq K$, such that

- either $U_{k}$ is a critical point of $f$ with index $p$,

- or $U_{k}$ is a critical point of $\left.f\right|_{\partial \Omega}$ with index $p-1$ such that $\partial_{n} f\left(U_{k}\right)>0$. For $\omega \in \Lambda^{p} H_{0, T}^{1}(\Omega)$ with $\|\omega\|_{\Lambda^{p} L^{2}, g}=1$, we get

$$
\left(\mathcal{D}_{g, f, h}(\omega) \leq C^{-1} h^{6 / 5}\right) \Rightarrow\left(\operatorname{dist}\left(\omega, \sum_{k \in A_{p}} F_{k}^{(p)}\right) \leq C h^{1 / 10}\right)
$$

Hence the dimension of the spectral subspace,

$$
F^{(p)}=\operatorname{Ran}_{\left[0, h^{3 / 2}\right)}\left(\Delta_{f, h}^{D T,(p)}\right) \subset \operatorname{Ran}_{\left[0, c h^{6 / 5}\right)}\left(\Delta_{f, h}^{D T,(p)}\right),
$$

is at most $\# A_{p}=m_{p}^{\bar{\Omega}}$.

We next verify that $\operatorname{dim} F^{(p)} \geq \# A_{p}=m_{p}^{\bar{\Omega}}$. According to the Min-Max principle, it suffices to find an orthonormal set of $p$-forms $\omega_{k}^{h} \in \Lambda^{p} H_{0, T}^{1}(\Omega)$, $k \in A_{p}$, such that

$$
\mathcal{D}_{g, f, h}\left(\omega_{k}^{h}\right)=o\left(h^{3 / 2}\right) .
$$

Indeed it is enough to take a truncated element of the kernel of the local model for $\Delta_{f, h}^{D T,(p)}$ around $U_{k}, k \in A_{p}$. We give the details for the case $U_{k} \in \partial \Omega$. By taking the same cut-off $\chi_{1, k}, \chi_{2, k}, \chi_{1, k}^{2}+\chi_{2, k}^{2}=1$, and the same coordinate system as above, we write on $\mathbb{R}_{-}^{n}$

$$
\mathcal{D}_{g_{k}, f_{k}, h}(\omega) \geq \mathcal{D}_{g_{k}, f_{k}, h}\left(\chi_{1, k} \omega\right)+C^{-1}\left\|\chi_{2, k} \omega\right\|^{2}-C h^{2} \sum_{i=1,2}\left\|\left|\nabla \chi_{i, k}\right| \omega\right\|^{2},
$$

where $g_{k}$ and $f_{k}$ are defined on $\mathbb{R}_{-}^{n}$ according to the previous construction and coincide with $g$ and $f$ in a neighborhood of $\operatorname{supp} \chi_{k}$. According to Proposition 3.13, there exists $\eta_{k}^{h} \in \Lambda^{p} H_{0, T}^{1}\left(\mathbb{R}_{-}^{n}\right)$ in the domain of the associated Witten Laplacian, such that $\mathcal{D}_{g_{k}, f_{k}, h}\left(\eta_{k}^{h}\right)=0$. By taking $\omega_{k}^{h}=\left\|\chi_{1, k} \eta_{k}^{h}\right\|^{-1} \chi_{k} \eta_{k}^{h}$, 
we obtain the existence of $h_{0}>0, C^{\prime}$ and $C^{\prime \prime}$ such that:

$$
\left\|\chi_{2, k} \eta_{k}^{h}\right\|^{2} \leq C^{\prime} h^{2}\left\|\eta_{k}^{h}\right\|^{2}
$$

and

$$
\mathcal{D}_{g, f, h}\left(\omega_{k}^{h}\right) \leq C^{\prime} h^{2} \frac{\left\|\eta_{k}^{h}\right\|^{2}}{\left\|\chi_{1, k} \eta_{k}^{h}\right\|^{2}} \leq C^{\prime \prime} h^{2}
$$

for $h \in\left(0, h_{0}\right]$.

\section{Accurate WKB analysis near the boundary for $\Delta_{f, h}^{(1)}$.}

\subsection{Preliminary discussion}

We work here under Assumption 3.1 while Assumption 3.8 will be satisfied for the local half-space model.

We have seen in the previous section that, for $p \geq 1$, some quasimodes of $\Delta_{f, h}^{D T,(p)}$ being near the spectral subspace in $1_{\left[0, h^{\frac{3}{2}}\right]}\left(\Delta_{f, h}^{D T,(p)}\right)$ are localized near the boundary $\partial \Omega$ and more precisely near critical points of $\left.f\right|_{\partial \Omega}$ with index $p-1$ such that $\partial_{n} f>0$. In the boundaryless case, the WKB-analysis done in [HelSj4] says that the small eigenvalues are of order $\mathcal{O}\left(e^{-C / h}\right)$ and provides an accurate approximate basis of $\operatorname{Ran} 1_{\left[0, h^{3 / 2}\right)}\left(\Delta_{f, h}^{(p)}\right)$.

In order to get a similar result, we need an accurate WKB analysis at the boundary in the spirit of the Helffer-Sjöstrand treatment of the tunneling effect in [HelSj1] and [He2]. Here again the boundary condition and the fact that we are working with systems for $p>0$ adds some technical difficulties. In an analytic framework, this could be attacked by studying the propagation of analytic regularity for microhyperbolic boundary value problems. At the boundary one has to consider first the tangential propagation of regularity and then the propagation into the interior. Having in mind our initial motivation of analyzing the Witten Laplacian on 0-forms, we shall study this problem with arguments as simple as possible and restrict our attention to the case $p=1$. Nevertheless, this "simple" presentation agrees with the general principle.

For an accurate comparison between eigenvectors and WKB quasimodes near 
a local minimum $U_{0}$ of $\left.f\right|_{\partial \Omega}$ with $\partial_{n} f\left(U_{0}\right)>0$, we introduce another selfadjoint realization of $\Delta_{f, h}^{(1)}$ in a neighborhood $\Omega_{U_{0}, \rho}$ with mixed boundary conditions : tangential Dirichlet boundary conditions on $\partial \Omega_{U_{0}, \rho} \cap \partial \Omega$ and full Dirichlet boundary on $\partial \Omega_{U_{0}, \rho} \backslash \partial \Omega$.

\subsection{Local WKB construction.}

The next construction is done locally around a local minimum $U_{0}$ of $\left.f\right|_{\partial \Omega}$ with $\partial_{n} f\left(U_{0}\right)>0$. The function $\Phi$ is a local solution of the eikonal equation $|\nabla \Phi|^{2}=|\nabla f|^{2}$, which satisfies (3.20) and (3.21). Local coordinates $\left(x^{\prime}, x_{n}\right)$ are introduced like in Subsection 3.4 after the identities (3.22)-(3.25) and lead to

$$
f\left(x^{\prime}, x_{n}\right)=x_{n}+\frac{1}{2} f_{-}\left(x^{\prime}\right) \text { and } \Phi\left(x^{\prime}, x_{n}\right)=-x_{n}+\frac{1}{2} f_{-}\left(x^{\prime}\right)
$$

with

$$
x_{n}<0 \text { in } \Omega \text { and } x_{n}=0 \text { on } \partial \Omega,
$$

and we normalize $f$ so that $f\left(U_{0}\right)=f(0)=f_{-}(0)=0$.

We first consider a local solution $u_{0}^{w k b}$ near the point $x=0$ of

$$
e^{\frac{\Phi}{h}} \Delta_{f}^{(0)} u_{0}^{w k b}=\mathcal{O}\left(h^{\infty}\right)
$$

with $u_{0}^{w k b}$ in the form

$$
\begin{gathered}
u_{0}^{w k b}=a(x, h) e^{-\frac{\Phi}{h}}, \\
a(x, h) \sim \sum_{j} a_{j}(x) h^{j},
\end{gathered}
$$

and the condition at the boundary

$$
a(x, h) e^{-\frac{\Phi}{h}}=e^{-\frac{f}{h}} \quad \text { on } \partial \Omega
$$

which leads to the condition

$$
\left.a(x, h)\right|_{\partial \Omega}=1 .
$$

This construction of $a$, as a solution of (4.1) in $\Omega$, (which can be first formal and then realized by using a Borel summation) is standard (see for example Chapter 2 (p. 11-12) in Dimassi-Sjöstrand [DiSj]). 
In order to verify locally the boundary condition for our future $u_{1}^{w k b}$, we substract $e^{-\frac{f}{h}}$ and still obtain

$$
e^{\frac{\Phi}{h}} \Delta_{f}^{(0)}\left(u_{0}^{w k b}-e^{-\frac{f}{h}}\right)=\mathcal{O}\left(h^{\infty}\right)
$$

We now define the WKB solution $u_{1}^{w k b}$ by considering :

$$
u_{1}^{w k b}:=d_{f, h} u_{0}^{w k b}=d_{f, h}\left(u_{0}^{w k b}-e^{-\frac{f}{h}}\right) .
$$

According to (4.4) and (4.7), the 1-form $u_{1}^{w k b}=d_{f, h} u_{0}^{w k b}$ satisfies locally the tangential condition $\mathbf{t} u=0$ on the boundary.

The local $L^{2}$ norm of $u_{1}^{w k b}$ is of effective order $h^{\frac{1}{2}+\frac{n-1}{4}}$, if one has in mind the relation

$$
\begin{aligned}
e^{\frac{\Phi}{h}} d_{f, h}\left[a(x, h) e^{-\frac{\Phi}{h}}\right] & =a(d f-d \Phi)+h d a \\
& =2 a_{0}(x) d x_{n}+h b^{1}(x, h)
\end{aligned}
$$

where $b^{1}(\cdot, h)$ is a one-form admitting the expansion :

$$
b^{1}(x, h) \sim \sum_{k=0}^{+\infty} h^{k} b_{k}(x)
$$

and $a_{0}$ satisfies :

$$
a_{0}(0)=1
$$

On the other hand we have

$$
\Delta_{f, h}^{(1)} u_{1}^{w k b}=\Delta_{f, h}^{(1)} d_{f, h} u_{0}^{w k b}=d_{f, h} \Delta_{f, h}^{(0)} u_{0}^{w k b}=\mathcal{O}\left(h^{\infty}\right) e^{-\frac{\Phi}{h}}
$$

in a neighborhood of 0 .

Moreover, $u_{1}^{w k b}$ satisfies up, to $\mathcal{O}\left(h^{\infty}\right) e^{-\frac{f}{h}}$, the second boundary condition $\mathbf{t} d_{f, h}^{*} u=0$. The relation

$$
d_{f, h}^{*} u_{1}^{w k b}=\Delta_{f, h}^{(0)} u_{0}^{w k b}=\mathcal{O}\left(h^{\infty}\right) e^{-\frac{\Phi}{h}}
$$

gives indeed

$$
\left.d_{f, h}^{*} u_{1}^{w k b}\right|_{/ \partial \Omega}=\mathcal{O}\left(h^{\infty}\right) e^{-\frac{f}{h}},
$$

in the neighborhood of 0 in the boundary. 


\subsection{Another local Dirichlet realization of $\Delta_{f, h}^{(1)}$.}

Let $U_{0}$ be a local minimum of $\left.f\right|_{\partial \Omega}$ with $\partial_{n} f\left(U_{0}\right)>0$, let $x=\left(x^{\prime}, x_{n}\right)$ be the local coordinate system defined above, and let $x \mapsto|x|$ be the euclidean norm in these coordinates.

For $\rho>0$, we consider the domain

$$
\Omega_{U_{0}, \rho}=\left\{|x-(0,1)|^{2}<\rho^{2}+1, x_{n}<0\right\},
$$

which has the shape of a thin lens stuck on $\partial \Omega$ with radius $\rho$ and thickness $\mathcal{O}\left(\rho^{2}\right)$. Its boundary is split into

$$
\Gamma_{D}:=\partial \Omega_{U_{0}, \rho} \cap \Omega=\left\{|x-(0,1)|^{2}=\rho^{2}+1, x_{n} \leq 0\right\},
$$

and

$$
\Gamma_{T D}:=\partial \Omega_{U_{0}, \rho} \cap \partial \Omega=\left\{\left|x^{\prime}\right|<\rho, x_{n}=0\right\} .
$$

On this domain, we introduce the functional space

$$
\Lambda^{1} H_{0 ; 0, T}^{1}\left(\Omega_{U_{0}, \rho}\right)=\left\{u \in \Lambda^{1} H^{1}\left(\Omega_{U_{0}, \rho}\right) ;\left.\mathbf{t} u\right|_{\Gamma_{T D}}=0,\left.\quad u\right|_{\Gamma_{D}}=0\right\} .
$$

The Friedrichs extension associated with the quadratic form :

$$
\Lambda^{1} H_{0 ; 0, T}^{1}\left(\Omega_{U_{0}, \rho}\right) \ni \omega \mapsto \mathcal{D}_{g, f, h}^{D}(\omega)=\left\|d_{f, h} \omega\right\|^{2}+\left\|d_{f, h}^{*} \omega\right\|^{2}
$$

is denoted by $\Delta_{f, h}^{D, D T,(1)}$. The domain of $\Delta_{f, h}^{D, D T,(1)}$ is contained in $\Lambda^{1} H^{2}\left(\Omega_{U_{0}}, \rho^{\prime}\right)$ for any $0<\rho^{\prime}<\rho$. An element $\omega \in D\left(\Delta_{f, h}^{D, D T,(1)}\right)$ satisfies indeed :

$\forall \eta \in \Lambda^{1} H_{0 ; 0, T}^{1},\left\langle\Delta_{f, h}^{D, D T,(1)} \omega \mid \eta\right\rangle=\left\langle d_{f, h} \omega \mid d_{f, h} \eta\right\rangle+\left\langle d_{f, h}^{*} \omega \mid d_{f, h}^{*} \eta\right\rangle=: \mathcal{D}_{g, f, h}^{D}(\omega, \eta)$.

By testing with $\eta \in \mathcal{C}_{0}^{\infty}\left(\Omega_{U_{0}, \rho}\right)$, this gives $\Delta_{f, h} \omega \in \Lambda^{1} L^{2}\left(\Omega_{U_{0}, \rho}\right)$ and therefore $\omega$ admits a second trace on $\Gamma_{T D}$. By testing with any $\eta \in \mathcal{C}_{0 ; 0, T}^{\infty}\left(\Omega_{U_{0, \rho}}\right)$, we get

$$
\left.\mathbf{t} d_{f, h}^{*} \omega\right|_{\Gamma_{T D}}=0
$$

Along $\Gamma_{T D}, \omega$ solves an elliptic boundary value problem $\Delta_{f, h}^{(1)} \omega \in \Lambda^{1} L^{2}$, $\mathbf{t} \omega=0, \mathbf{t} d_{f, h}^{*} \omega=0$, which provides the $H^{2}$ regularity outside the edges. 
Remark 4.1. It is actually possible to characterize the domain, for $\rho>0$ small enough, by :

$$
\begin{array}{r}
D\left(\Delta_{f, h}^{D, D T,(1)}\right)=\left\{u \in \Lambda^{1} H^{2}\left(\Omega_{U_{0}}, \rho\right),\left.\mathbf{t} u\right|_{\Gamma_{T D}}=0,\left.\mathbf{t} d_{f, h}^{*} u\right|_{\Gamma_{T D}}=0,\right. \\
\text { and } \left.\left.u\right|_{\Gamma_{D}}=0 .\right\}
\end{array}
$$

For this regularity result it suffices to consider the case $f=0$ and $h=1$. The boundary conditions are written for $u=u_{1} d x_{1}+\cdots+u_{n} d x_{n}$ in the form

$$
\begin{aligned}
& \left.u_{i}\right|_{\partial \Omega_{U_{0}, \rho}}=0, \quad \text { for } i=1, \ldots, n-1, \\
& \left.u_{n}\right|_{\Gamma_{D}}=0,\left.\quad \partial_{x_{n}} u_{n}\right|_{\Gamma_{T D}}=0,
\end{aligned}
$$

while the principal part of $\Delta_{f, h}^{(1)}$ is a scalar Laplace operator, as can be seen from the Weitzenböck formula. Hence componentwise and at the principal symbol level, the most difficult case is a Dirichlet-Neumann problem for the operator $\sum_{j=1}^{n} \partial_{x_{i}} g^{i j} \partial_{x_{j}}$ according to (3.9) and (3.10). The theory of boundary value problems on domains with conical singularities ([Kon]) and edges ([Gri], [Da]) provides the $H^{2}$-regularity when $\rho<\rho_{\text {lim }}$, where $\rho_{\text {lim }}$ can be computed explicitly ( $\rho_{\text {lim }}=\pi / 2$ for this mixed Dirichlet-Neumann problem). Notice that we do not need this result and that the $H^{2}$-regularity away from the edge is sufficient for our analysis.

We now prove the

\section{Proposition 4.2.}

For $\rho>0$ small enough, there exist $h_{\rho}>0$ and $C_{\rho}>0$, such that the selfadjoint operator $\Delta_{f, h}^{D, D T,(1)}$ satisfies the following properties :

a) For $h \in\left(0, h_{\rho}\right)$, the spectral projection $1_{\left[0, h^{3 / 2}\right)}\left(\Delta_{f, h}^{D, D T,(1)}\right)$ has rank 1 .

b) Any family of $L^{2}$-normalized eigenvectors $\left(u^{h}\right)_{h \in\left(0, h_{\rho}\right)}$ of $\Delta_{f, h}^{D, D T,(1)}$ such that the corresponding eigenvalue $E(h)$ is $\mathcal{O}(h)$, satisfies

$$
\begin{aligned}
& \forall \rho^{\prime}<\rho, \forall \alpha \in \mathbb{N}^{d}, \exists N_{\alpha} \in \mathbb{N}, \exists C_{\alpha, \rho^{\prime}}>0 \text { such that, } \forall x \in \Omega_{U_{0}, \rho^{\prime}} \\
& \left|\partial_{x}^{\alpha} u^{h}(x)\right| \leq C_{\alpha, \rho^{\prime}} h^{-N_{\alpha}} \exp \left(-\frac{\Phi(x)}{h}\right) .
\end{aligned}
$$

c) There exists $\varepsilon_{\rho}>0$ such that the first eigenvalue $E_{1}(h)$ of $\Delta_{f, h}^{D, D T,(1)}$ satisfies

$$
E_{1}(h)=e^{-\varepsilon_{\rho} / h}
$$


d) If $u_{1}^{h}$ denotes the eigenvector of $\Delta_{f, h}^{D, D T,(1)}$ associated to eigenvalue $E_{1}(h)$ and normalized by the condition $\mathbf{i}_{\partial_{x_{n}}} u_{1}^{h}(0)=\mathbf{i}_{\partial_{x_{n}}} u_{1}^{w k b}(0)$, then

$$
\begin{aligned}
& \forall \rho^{\prime}<\rho, \forall \alpha \in \mathbb{N}^{d}, \forall N \in \mathbb{N}, \exists C_{N, \alpha, \rho^{\prime}}>0 \text { such that }, \forall x \in \Omega_{U_{0}, \rho^{\prime}}, \\
& \left|\partial_{x}^{\alpha}\left(u_{1}^{h}-u_{1}^{w k b}\right)(x)\right| \leq C_{N, \alpha, \rho^{\prime}} h^{N} \exp \left(-\frac{\Phi(x)}{h}\right) .
\end{aligned}
$$

Once this is proved, one easily gets rough exponentially small upper bounds for the $m_{\ell}^{\bar{\Omega}}$ first eigenvalues of $\Delta_{f, h}^{D T,(\ell)}(\ell \in\{0,1\})$ on $\Omega$, by constructing quasimodes suitably localized near each of the critical points.

Our final analysis provides the exact exponential scale with a complete expansion of the prefactor, and we do not develop this point here.

The next subsections are devoted to the proof of Proposition 4.2. We now introduce some specific notations and preliminary results. Again with the coordinate system $\left(x^{\prime}, x_{n}\right)$ with $x^{\prime}\left(U_{0}\right)=0, x_{n}\left(U_{0}\right)=0$, and the normalization $f(0)=0$, the function $\left.f\right|_{\Omega_{U_{0}, \rho_{0}}}$ is extended to $\overline{\mathbb{R}_{-}^{n}}$ according to Lemma 3.11, so that Assumption 3.5 is satisfied with only one tangential critical point at $x^{\prime}=0$. The corresponding tangential Dirichlet realization $\Delta_{\tilde{f}, h}^{D T,(1)}$ on $\Lambda^{1} L^{2}\left(\mathbb{R}_{-}^{n}\right)$ has a 1-dimensional kernel and its second eigenvalue is larger than $C^{-1} h^{6 / 5}$.

An ingredient for the proof is a variant of the integration by part formula of Lemma 2.2.

Lemma 4.3.

Let $\rho>0$ and let $\varphi$ be a real-valued Lipschitz function on $\overline{\Omega_{U_{0}, \rho}}$. The relation

$$
\begin{aligned}
\operatorname{Re} \mathcal{D}_{g, f, h}^{D}\left(\omega, e^{2 \frac{\varphi}{h}} \omega\right)=h^{2}\left\|d e^{\frac{\varphi}{h}} \omega\right\|_{\Lambda^{2} L^{2}}^{2}+h^{2}\left\|d^{*} e^{\frac{\varphi}{h}} \omega\right\|_{\Lambda^{0} L^{2}}^{2} \\
+\left\langle\left(|\nabla f|^{2}-|\nabla \varphi|^{2}+h \mathcal{L}_{\nabla f}+h \mathcal{L}_{\nabla f}^{*}\right) e^{\frac{\varphi}{h} \omega\left|e^{\frac{\varphi}{h}} \omega\right\rangle_{\Lambda^{1} L^{2}}}\right. \\
-h \int_{\Gamma_{T D}}\langle\omega \mid \omega\rangle_{\Lambda^{1} T_{\sigma}^{*} \Omega} e^{2 \frac{\varphi(\sigma)}{h}}\left(\frac{\partial f}{\partial n}\right)(\sigma) d \sigma
\end{aligned}
$$

holds for any $\omega \in \Lambda^{1} H_{0 ; 0, T}^{1}\left(\Omega_{U_{0}, \rho}\right)$. Moreover, when $\omega \in D\left(\Delta_{f, h}^{D, D T,(1)}\right)$, the left-hand side equals $\operatorname{Re}\left\langle e^{2 \frac{\varphi}{h}} \Delta_{f, h}^{(1)} \omega \mid \omega\right\rangle$.

Proof. 
For $\omega \in \Lambda^{1} H_{0 ; 0, T}^{1}\left(\Omega_{U_{0, \rho}}\right)$, we have $e^{2 \frac{\varphi}{h}} \omega \in \Lambda^{1} H_{0 ; 0, T}^{1}\left(\Omega_{U_{0}, \rho}\right)$. We compute

$$
\begin{aligned}
\mathcal{D}_{g, f, h}^{D}\left(\omega, e^{2 \frac{\varphi}{h}} \omega\right)= & \left\langle d_{f, h} \omega \mid d_{f, h}\left(e^{2 \frac{\varphi}{h}} \omega\right)\right\rangle+\left\langle d_{f, h}^{*} \omega \mid d_{f, h}^{*}\left(e^{2 \frac{\varphi}{h}} \omega\right)\right\rangle \\
= & \left\langle d_{f, h} \omega \mid e^{\frac{\varphi}{h}} d_{f, h}\left(e^{\frac{\varphi}{h}} \omega\right)\right\rangle+\left\langle e^{\frac{\varphi}{h}} d_{f, h} \omega \mid d \varphi \wedge\left(e^{\frac{\varphi}{h}} \omega\right)\right\rangle \\
& +\left\langle d_{f, h}^{*} \omega \mid e^{\frac{\varphi}{h}} d_{f, h}^{*}\left(e^{\frac{\varphi}{h}} \omega\right)\right\rangle-\left\langle e^{\frac{\varphi}{h}} d_{f, h}^{*} \omega \mid \mathbf{i}_{\nabla \varphi}\left(e^{\frac{\varphi}{h}} \omega\right)\right\rangle \\
= & \left\langled _ { f , h } \left( e^{\frac{\varphi}{h}} \omega\left|d_{f, h}\left(e^{\frac{\varphi}{h}} \omega\right)\right\rangle-\left\langle d \varphi \wedge\left(e^{\frac{\varphi}{h}} \omega\right) \mid d_{f, h}\left(e^{\frac{\varphi}{h}} \omega\right)\right\rangle\right.\right. \\
& +\left\langle d_{f, h}\left(e^{\frac{\varphi}{h}} \omega\right) \mid d \varphi \wedge\left(e^{\frac{\varphi}{h}} \omega\right)\right\rangle-\left\langle d \varphi \wedge\left(e^{\frac{\varphi}{h}} \omega\right) \mid d \varphi \wedge\left(e^{\frac{\varphi}{h}} \omega\right)\right\rangle \\
& +\left\langle d_{f, h}^{*}\left(e^{\frac{\varphi}{h}} \omega\right) \mid d_{f, h}^{*}\left(e^{\frac{\varphi}{h}} \omega\right)\right\rangle+\left\langle\mathbf{i}_{\nabla \varphi}\left(e^{\frac{\varphi}{h}} \omega\right) \mid d_{f, h}^{*}\left(e^{\frac{\varphi}{h}} \omega\right)\right\rangle \\
& -\left\langle d_{f, h}^{*}\left(e^{\frac{\varphi}{h} \omega}\right) \mid \mathbf{i}_{\nabla \varphi}\left(e^{\frac{\varphi}{h}} \omega\right)\right\rangle-\left\langle\mathbf{i}_{\nabla \varphi}\left(e^{\frac{\varphi}{h}} \omega\right) \mid \mathbf{i}_{\nabla \varphi}\left(e^{\frac{\varphi}{h}} \omega\right)\right\rangle .
\end{aligned}
$$

We set $\tilde{\omega}=e^{\frac{\varphi}{h} \omega} \in \Lambda^{1} H_{0 ; 0, T}^{1}\left(\Omega_{U_{0}, \rho}\right)$. The operator $d \varphi \wedge$ is the adjoint of $\mathbf{i}_{\nabla \varphi}$ and the tensor relation

$$
\mathbf{i}_{\nabla \varphi}(d \varphi \wedge \eta)=\left(\mathbf{i}_{\nabla \varphi} d \varphi\right) \wedge \eta-d \varphi \wedge\left(\mathbf{i}_{\nabla \varphi} \eta\right)=|\nabla \varphi|^{2} \eta-d \varphi \wedge\left(\mathbf{i}_{\nabla \varphi} \eta\right)
$$

leads to

$$
\begin{aligned}
\mathcal{D}_{g, f, h}^{D}\left(\omega, e^{2 \frac{\varphi}{h}} \omega\right)= & \mathcal{D}_{g, f, h}^{D}(\tilde{\omega}, \tilde{\omega})-\left\langle|\nabla \varphi|^{2} \tilde{\omega} \mid \tilde{\omega}\right\rangle \\
& -\left\langle d \varphi \wedge \tilde{\omega} \mid d_{f, h} \tilde{\omega}\right\rangle+\left\langle d_{f, h} \tilde{\omega} \mid d \varphi \wedge \tilde{\omega}\right\rangle \\
& +\left\langle\mathbf{i}_{\nabla \varphi} \tilde{\omega} \mid d_{f, h}^{*} \tilde{\omega}\right\rangle-\left\langle d_{f, h}^{*} \tilde{\omega} \mid \mathbf{i}_{\nabla \varphi} \tilde{\omega}\right\rangle .
\end{aligned}
$$

After taking the real part, we obtain

$$
\operatorname{Re} \mathcal{D}_{g, f, h}^{D}\left(\omega, e^{2 \frac{\varphi}{h}} \omega\right)=\mathcal{D}_{g, f, h}^{D}(\tilde{\omega}, \tilde{\omega})-\left\langle|\nabla \varphi|^{2} \tilde{\omega} \mid \tilde{\omega}\right\rangle .
$$

We conclude by applying Lemma 2.2 .

\subsection{Exponential decay of eigenvectors of $\Delta_{f, h}^{D, D T,(1)}$.}

The estimate, $\partial_{x}^{\alpha} u^{h}(x)=\mathcal{O}\left(h^{-N \alpha} e^{-\frac{\Phi(x)}{h}}\right)$, which is stated in Proposition 4.2b), will be proved in several steps. We will first consider $H^{1}$-estimates and deduce afterwards higher order estimates from elliptic regularity. Even for $H^{1}$-estimates we need two steps :

1) We prove the exponential decay along the boundary $\Gamma_{T D}$ by applying Lemma 4.3 with a function $\varphi$ similar to $\frac{1}{2} f_{-}$.

2) The exponential decay in the interior of $\Omega_{U_{0}, \rho}$ is then obtained with $\varphi$ similar to $\Phi$ once the boundary term is well controlled. 
Proof of a) and b) in Proposition 4.2.

\section{Statement a)}

Actually it is a simple comparison with the full half-space problem via Min-

Max principle as we did for Theorem 3.3. Any $\omega \in \Lambda^{1} H_{0 ; 0, T}^{1}\left(\Omega_{U_{0}, \rho}\right)$ can indeed be viewed as an element of $\Lambda^{1} H_{0, T}^{1}\left(\mathbb{R}_{-}^{n}\right)$ by setting $\omega=0$ on $\mathbb{R}_{-}^{n} \backslash \Omega_{U_{0}, \rho}$.

\section{Statement b)}

Let $u^{h} \in D\left(\Delta_{f, h}^{D, D T,(1)}\right)$ satisfy

$$
\Delta_{f, h}^{(1)} u^{h}=E(h) u^{h}, E(h)=\mathcal{O}(h),\left\|u^{h}\right\|=1 .
$$

We will use the notation

$$
\tilde{u}^{h}=e^{\frac{\varphi^{h}}{h}} u^{h} .
$$

The integration by part formula (4.11) will be applied with $\varphi=\varphi^{h}$, where with $\varphi^{h}$ similar to $\frac{1}{2} f_{-}$or $\varphi^{h}$ similar to $\Phi$. We recall

$$
f\left(x^{\prime}, x_{n}\right)=x_{n}+\frac{1}{2} f_{-}\left(x^{\prime}\right) \text { and } \Phi\left(x^{\prime}, x_{n}\right)=-x_{n}+\frac{1}{2} f_{-}\left(x^{\prime}\right),
$$

where $x^{\prime}=0$ is a local minimum for $f_{-}$with $f_{-}(0)=0$. Moreover we have $\nabla x_{n} \cdot \nabla f_{-}\left(x^{\prime}\right)=0$, so that :

$$
|\nabla f|^{2}=\left|\nabla x_{n}\right|^{2}+\frac{1}{4}\left|\nabla f_{-}\right|^{2}
$$

The proof which follows is somewhat reminiscent of [HelSj5], which was dealing with Schrödinger operators with miniwells. We will first show the decay along the boundary before we "propagate" the decay in the normal direction inside $\Omega$.

Step 1 : Decay along $\Gamma_{T D}$.

We take

$$
\varphi^{h}(x)=\frac{1}{2} \varphi_{-}^{h}\left(x^{\prime}\right),
$$

with

$$
\varphi_{-}^{h}\left(x^{\prime}\right)= \begin{cases}f_{-}\left(x^{\prime}\right)-C h \log \frac{f_{-}\left(x^{\prime}\right)}{h}, & \text { if } f_{-}\left(x^{\prime}\right)>C h, \\ f_{-}\left(x^{\prime}\right)-C h \log C, & \text { if } f_{-}\left(x^{\prime}\right) \leq C h,\end{cases}
$$

where the constant $C>1$ will be fixed later. We associate the sets

$$
\Omega_{-}^{h}=\left\{x=\left(x^{\prime}, x_{n}\right) \in \Omega_{U_{0}, \rho} ; f_{-}\left(x^{\prime}\right)<C h\right\},
$$

and

$$
\Omega_{+}^{h}=\left\{x=\left(x^{\prime}, x_{n}\right) \in \Omega_{U_{0}, \rho} ; f_{-}\left(x^{\prime}\right)>C h\right\} .
$$


The condition $E(h)=\mathcal{O}(h)$, formula (4.11), and the equality $\left|\nabla \varphi^{h}\right|=\frac{1}{2}|\nabla f|$ in $\Omega_{-}^{h}$ imply the existence of $C_{1}>0$ such that :

$$
\begin{aligned}
C_{1} h\left\|\tilde{u}^{h}\right\|_{\Lambda^{1} L^{2}\left(\Omega_{-}^{h}\right)}^{2} \geq & \left\|h d \tilde{u}^{h}\right\|_{\Lambda^{2} L^{2}}^{2}+\left\|h d^{*} \tilde{u}^{h}\right\|_{\Lambda^{0} L^{2}}^{2}+\left\langle\left|\nabla x_{n}\right|^{2} \tilde{u}^{h} \mid \tilde{u}^{h}\right\rangle_{\Lambda^{1} L^{2}} \\
& \quad-h \int_{\Gamma_{T D}}\left\langle\tilde{u}^{h} \mid \tilde{u}^{h}\right\rangle_{\Lambda^{1} T_{\sigma}^{*} \Omega}\left(\frac{\partial x_{n}}{\partial n}\right)(\sigma) d \sigma \\
& +\frac{1}{4}\left\langle\left(\left|\nabla f_{-}\right|^{2}-\left|\nabla \varphi_{-}^{h}\right|^{2}-4 C_{1} h\right) 1_{\Omega_{+}^{h}}(x) \tilde{u}^{h} \mid \tilde{u}^{h}\right\rangle,
\end{aligned}
$$

with $C_{1}$ determined by $f$ and the upper bound of $E(h)$.

For $x \in \Omega_{-}^{h}$, one immediately gets from the definitions that

$$
\left|\tilde{u}^{h}(x)\right| \leq \exp \frac{C}{2}\left|u^{h}(x)\right| \quad \text { a.e. } .
$$

We obtain, for a constant $C_{2}(C)$ which may depend on the choice of $C$,

$$
\begin{aligned}
C_{2}(C) h & \geq\left\|h d \tilde{u}^{h}\right\|_{\Lambda^{2} L^{2}}^{2}+\left\|h d^{*} \tilde{u}^{h}\right\|_{\Lambda^{0} L^{2}}^{2} \\
& +\left\langle\left|\nabla x_{n}\right|^{2} \tilde{u}^{h} \mid \tilde{u}^{h}\right\rangle_{\Lambda^{1} L^{2}}-h \int_{\Gamma_{T D}}\left\langle\tilde{u}^{h} \mid \tilde{u}^{h}\right\rangle_{\Lambda^{1} T_{\sigma}^{*} \Omega}\left(\frac{\partial x_{n}}{\partial n}\right)(\sigma) d \sigma \\
+ & \frac{1}{4}\left\langle\left(\left|\nabla f_{-}\right|^{2}-\left|\nabla \varphi_{-}^{h}\right|^{2}-4 C_{1} h\right) 1_{\Omega_{+}^{h}}(x) \tilde{u}^{h} \mid \tilde{u}^{h}\right\rangle+C_{1} h\left\langle 1_{\Omega_{-}^{h}}(x) \tilde{u}^{h} \mid \tilde{u}^{h}\right\rangle .
\end{aligned}
$$

For $x \in \Omega_{+}^{h}$, we write

$$
\nabla \varphi_{-}^{h}\left(x^{\prime}\right)=\nabla f_{-}\left(x^{\prime}\right)\left(1-\frac{C h}{f_{-}\left(x^{\prime}\right)}\right)
$$

and

$$
\frac{1}{4}\left(\left|\nabla f_{-}\right|^{2}-\left|\nabla \varphi_{-}^{h}\right|^{2}\right)=\frac{\left|\nabla f_{-}\right|^{2}}{4}\left(\frac{2 C h}{f_{-}}-\frac{C^{2} h^{2}}{f_{-}^{2}}\right) \geq C h \frac{\left|\nabla f_{-}\right|^{2}}{4 f_{-}} .
$$

Since there exists $C_{4}>0$, which is determined by $f_{-}$, such that

$$
C_{4} \geq \frac{\left|\nabla f_{-}\left(x^{\prime}\right)\right|^{2}}{4 f_{-}\left(x^{\prime}\right)} \geq C_{4}^{-1}
$$

we get

$$
\forall x \in \Omega_{+}^{h}, \quad \frac{1}{4}\left(\left|\nabla f_{-}(x)\right|^{2}-\left|\nabla \varphi_{-}^{h}(x)\right|^{2}\right)-C_{1} h \geq\left(\frac{C}{C_{4}}-C_{1}\right) h .
$$


We obtain for any $C \geq \max \left(1,2 C_{1} C_{4}\right)$, the existence of $\delta(C)>0$ and $C_{3}(C)>0$ such that:

$$
\begin{aligned}
C_{3}(C) h \geq \| & h d \tilde{u}^{h}\left\|_{\Lambda^{2} L^{2}}^{2}+\right\| h d^{*} \tilde{u}^{h} \|_{\Lambda^{0} L^{2}}^{2} \\
& +(1+2 \delta(C) h)\left\langle\left|\nabla x_{n}\right|^{2} \tilde{u}^{h} \mid \tilde{u}^{h}\right\rangle_{\Lambda^{1} L^{2}} \\
& -h \int_{\Gamma_{T D}}\left\langle\tilde{u}^{h} \mid \tilde{u}^{h}\right\rangle_{\Lambda^{1} T_{\sigma}^{*} \Omega}\left(\frac{\partial x_{n}}{\partial n}\right)(\sigma) d \sigma
\end{aligned}
$$

with $\lim _{C \rightarrow+\infty} \delta(C)=+\infty$.

We now can use (4.11), with $\varphi=0, f=x_{n}, \hat{h}=\frac{h}{1+\delta(C) h}$, in order to get, for all $\eta \in \Lambda^{1} H_{0 ; 0, T}^{1}\left(\Omega, U_{0, \rho}\right)$,

$$
\begin{aligned}
& (1+\delta h)^{-1}\|h d \eta\|_{\Lambda^{2} L^{2}}^{2}+(1+\delta h)^{-1}\left\|h d^{*} \eta\right\|_{\Lambda^{0} L^{2}}^{2} \\
+ & (1+\delta h)\left\langle\left|\nabla x_{n}\right|^{2} \eta \mid \eta\right\rangle-h \int_{\Gamma_{T D}}\langle\eta \mid \eta\rangle_{\Lambda^{1} T_{\sigma}^{*} \Omega}\left(\frac{\partial x_{n}}{\partial n}\right)(\sigma) d \sigma \geq-h \widehat{C}\|\eta\|_{\Lambda^{1} L^{2}}^{2}
\end{aligned}
$$

with $\delta=\delta(C)$ and $\widehat{C}$ independent of $C$.

This leads, by choosing $C$ large enough and then $h_{0}>0$ small enough, to the existence of a constant $C_{5}>0$ such that, for all $h \in\left(0, h_{0}\right]$,

$$
C_{5} h \geq \frac{1}{C_{5}} h^{3}\left\|\tilde{u}^{h}\right\|_{\Lambda^{1} H^{1}}^{2}
$$

Since $\varphi^{h} \geq \frac{1}{2} f_{-}+C_{6} h \log h$, we have proved the existence of $N_{0}>0$ such that :

$$
\left\|e^{\frac{f_{-}}{2 h}} u^{h}\right\|_{\Lambda^{1} H^{1}} \leq C_{7} h^{-N_{0}}
$$

where $x^{\prime}=0$ is a local minimum for $f_{-}$, with $f_{-}(0)=0$. Note that, since $\left.f\right|_{\Gamma_{T D}}=\frac{1}{2} f_{-}$, this implies also, using the trace theorem,

$$
\left\|\left.e^{\frac{f}{h}} u^{h}\right|_{\Gamma_{T D}}\right\|_{\Lambda^{1} H^{1 / 2}\left(\Gamma_{T D}\right)} \leq C_{8} h^{-N_{0}}
$$

Step 2 : Normal decay inside $\Omega$.

We follow a similar approach by working with the normal coordinate $x_{n}$. We take

$$
\varphi^{h}(x)=\frac{1}{2} \varphi_{+}^{h}\left(x_{n}\right)+\frac{1}{2} f_{-}\left(x^{\prime}\right)
$$


with

$$
\varphi_{+}^{h}\left(x_{n}\right)= \begin{cases}2\left|x_{n}\right|-C h \log \frac{2\left|x_{n}\right|}{h}, & \text { if } 2\left|x_{n}\right|>C h, \\ 2\left|x_{n}\right|-C h \log C, & \text { if } 2\left|x_{n}\right| \leq C h\end{cases}
$$

where the constant $C \geq 1$ will be fixed later. We associate the sets

$$
\Omega_{-}^{h}=\left\{x=\left(x^{\prime}, x_{n}\right) \in \Omega_{U_{0}, \rho} ; 2\left|x_{n}\right|<C h\right\}
$$

and

$$
\Omega_{+}^{h}=\left\{x=\left(x^{\prime}, x_{n}\right) \in \Omega_{U_{0}, \rho} ; 2\left|x_{n}\right|>C h\right\} .
$$

The formula (4.11) is used like in Step 1, with $\tilde{u}^{h}=e^{\frac{\varphi^{h}}{h}} u^{h}$ and $E(h)=$ $\mathcal{O}(h)$. The difference comes from the fact that the boundary term is already estimated with (4.13).

We have indeed, on the boundary $x_{n}=0$, the inequality : $e^{\frac{\varphi^{h}}{h}} \leq e^{\frac{f}{h}}$. From (4.11), (4.12), (4.13), and the inequality

$$
\left|\tilde{u}^{h}(x)\right| \leq e^{C} e^{\frac{f_{-}(x)}{2 h}}\left|u^{h}(x)\right|, \quad \text { a.e. in } \Omega_{-}^{h},
$$

we get, for a $C$-dependent constant $C_{2}(C)$, the estimate

$$
\begin{aligned}
& C_{2}(C)^{2} h^{-2 N_{0}} \geq C_{1} h\left\|\tilde{u}^{h}\right\|_{\Lambda^{1} L^{2}\left(\Omega_{-}^{h}\right)}^{2}+C_{1} h\left\|e^{\frac{f}{h}} u\right\|_{H^{1 / 2}\left(\Gamma_{T D} ; \Lambda^{1} T^{*} \Omega_{U_{0}, \rho}\right)}^{2} \\
& \geq\left\|h d \tilde{u}^{h}\right\|_{\Lambda^{2} L^{2}}^{2}+\left\|h d^{*} \tilde{u}^{h}\right\|_{\Lambda^{0} L^{2}}^{2}+\left\langle\left(|\nabla f|^{2}-\left|\nabla \varphi^{h}\right|^{2}-C_{1} h\right) 1_{\Omega_{+}^{h}}(x) \tilde{u}^{h} \mid \tilde{u}^{h}\right\rangle,
\end{aligned}
$$

with $C_{1}>0$ independent of $C \geq 1$.

For $x \in \Omega_{+}^{h}$ we have

$$
\nabla \varphi_{+}^{h}=-2\left(\nabla x_{n}\right)\left(1-\frac{C h}{\left|x_{n}\right|}\right)
$$

and

$$
|\nabla f|^{2}-\left|\nabla \varphi^{h}\right|^{2} \geq\left|\nabla x_{n}\right|^{2}-\frac{1}{4}\left|\nabla \varphi_{+}^{h}\right|^{2} \geq \frac{C h}{4 C_{3}\left|x_{n}\right|} .
$$

We can assume $\left|x_{n}\right| \leq 1$ in $\Omega_{U_{0}, \rho}$ and we take $C \geq 8 C_{1} C_{3}$. The conclusion is simpler than in Step 1. By adding the estimated term $C_{1} h\left\langle\tilde{u}^{h} 1_{\Omega_{-}^{h}}(x) \mid \tilde{u}^{h}\right\rangle$, we get

$$
C_{4} h^{-2 N_{0}} \geq\left\|h d \tilde{u}^{h}\right\|_{\Lambda^{2} L^{2}}^{2}+\left\|h d^{*} \tilde{u}^{h}\right\|_{\Lambda^{0} L^{2}}^{2}+C_{1} h\left\|\tilde{u}^{h}\right\|_{\Lambda^{1} L^{2}}
$$


which gives the existence of $C_{5}$ and $N_{1}$ such that :

$$
\left\|e^{\frac{\Phi}{h}} u^{h}\right\|_{\Lambda^{1} H^{1}\left(\Omega_{U_{0}}, \rho\right)} \leq C_{5} h^{-N_{1}} .
$$

Step 3 : Elliptic regularity.

We now set $\tilde{u}^{h}=e^{\frac{\Phi}{h}} u^{h}$. For $\rho^{\prime} \subset \rho$, we take a cut-off $\chi \in \mathcal{C}^{\infty}\left(\Omega_{U_{0}, \rho}\right)$ with compact support in $\Omega_{U_{0}, \rho} \cup \Gamma_{T D}$ and such that $\chi=1$ on a neighborhood of $\Omega_{U_{0}, \rho^{\prime}}$. The form $v^{h}=\chi \tilde{u}^{h}$ satisfies the boundary value problem

$$
\begin{gathered}
\begin{cases}v^{h}-\Delta v^{h}=r_{0}^{h} & \text { in } \mathbb{R}_{-}^{n}, \\
\mathbf{t} v^{h}=0 \text { and } \mathbf{t} d^{*} v^{h}=r_{1}^{h} & \text { on }\left\{x_{n}=0\right\},\end{cases} \\
\text { with }\left\|r_{0}^{h}\right\|_{\Lambda^{1} L^{2}\left(\mathbb{R}_{-}^{n}\right)}=\mathcal{O}\left(h^{-N_{1}}\right) \text { and }\left\|r_{1}^{h}\right\|_{\Lambda^{0} H^{1 / 2}\left(\mathbb{R}^{n-1}\right)}=\mathcal{O}\left(h^{-N_{1}}\right) .
\end{gathered}
$$

This implies the existence of $N_{1}>0$ such that:

$$
\left\|v^{h}\right\|_{\Lambda^{1} H^{2}}=\mathcal{O}\left(h^{-N_{1}}\right) .
$$

We conclude by induction for any finite decreasing sequence $\left(\rho_{k}\right)_{0 \leq k \leq K}$ and associated cut-offs $\chi_{k}$, with $\chi_{k}=1$ in a neighborhood of $\Omega_{U_{0}, \rho_{k}}$ and supp $\chi_{k} \subset$ $\left\{\chi_{k-1}=1\right\}$.

\subsection{Small eigenvalues are exponentially small.}

We now check that the eigenvalue $E_{1}(h)$ of $\Delta_{f, h}^{D, D T,(1)}$ lying in $\left[0, h^{3 / 2}\right)$ is actually of order $\mathcal{O}\left(e^{-\varepsilon_{\rho} / h}\right)$, for some $\varepsilon_{\rho}>0$. We prove this by comparing with the half-space problem, for which we know that the first eigenvalue is 0. The Min-Max principle or Lemma 2.8 are not sufficient here and we need the full accuracy of the spectral theorem.

Proof of Proposition 4.2-c).

We assume that $\rho>0$ is small enough, so that $f$ admits an extension $\tilde{f}=x_{n}+\frac{1}{2} \tilde{f}_{-}\left(x^{\prime}\right)$ on $\mathbb{R}_{-}^{n}$, which satisfies Assumption 3.8. So the Laplacian $\Delta_{\tilde{f}, h}^{D T,(1)}$ has a one dimension kernel and its second eigenvalue is larger than $C h^{6 / 5}$. With this function $\tilde{f}$, we associate the second solution of the eikonal equation $|\nabla \tilde{\Phi}|^{2}=|\nabla \tilde{f}|^{2}$, which has the expression :

$$
\tilde{\Phi}(x)=-x_{n}+\frac{1}{2} \tilde{f}_{-}\left(x^{\prime}\right) .
$$


Let $u^{h}$ be a normalized eigenvector of $\Delta_{f, h}^{D, D T,(1)}$ associated with the first eigenvalue $E_{1}(h)$, which belongs to the interval $\left(0, h^{3 / 2}\right]$. Let $\chi \in \mathcal{C}^{\infty}\left(\overline{\Omega_{U_{0}, \rho}}\right)$ be a cut-off function with compact support in $\Omega_{U_{0}, \rho} \cup \Gamma_{T D}$ and such that $\chi=1$ in a neighborhood of 0 . The form $v^{h}=\chi u^{h}$ belongs to $\Lambda^{1} H^{2}\left(\mathbb{R}_{-}^{n}\right)$ and satisfies

$$
\begin{cases}\left(\Delta_{\tilde{f}, h}^{(1)}-E_{1}(h)\right) v^{h}=-h^{2}[\Delta, \chi] u^{h}, & \text { in } \mathbb{R}_{-}^{n}, \\ \mathbf{t} v^{h}=0, \quad \mathbf{t} d_{\tilde{f}, h}^{*} v^{h}=\mathbf{i}_{\nabla \chi} u^{h}, & \text { on }\left\{x_{n}=0\right\}\end{cases}
$$

The functions $r_{0}^{h}=-h^{2}[\Delta, \chi] u^{h}$ and $r_{1}^{h}=\mathbf{i}_{\nabla \chi} u^{h}$ vanish in a neighborhood $\mathcal{V}_{1}$ of $x=0$. Due to the exponential decay of $u^{h}$ stated in Proposition 4.2-b), there exist $C$ and $N_{0}$, such that they also satisfy

$$
\left|r_{j}^{h}(x)\right| \leq C h^{-N_{0}} e^{-\frac{\tilde{\Phi}(x)}{h}} .
$$

Due to the trace theorem, it is possible to find $\tilde{\theta}^{h} \in \Lambda^{1} H^{2}\left(\mathbb{R}_{-}^{n}\right)$, such that

$$
\mathbf{t} \tilde{\theta}^{h}=0 \quad \text { and } \quad \mathbf{t} d^{*} \tilde{\theta}^{h}=e^{\frac{\tilde{f}(x)}{h}} \mathbf{i}_{\nabla \chi} u^{h}
$$

with, using the property $\left.\tilde{\Phi}\right|_{\left\{x_{n}=0\right\}}=\left.\tilde{f}\right|_{\left\{x_{n}=0\right\}}$,

$$
\left\|\tilde{\theta}^{h}\right\|_{\Lambda^{1} H^{2}} \leq C h^{-N_{0}}
$$

Moreover by possibly taking a smaller neighborhood $\mathcal{V}_{1}$, the forms $\theta$ and $\tilde{\theta}$ can be chosen so that $\operatorname{supp} \theta \cap \mathcal{V}_{1}=\operatorname{supp} \tilde{\theta} \cap \mathcal{V}_{1}=\emptyset$.

For any given neighborhood of $0, \mathcal{V}_{2} \subset \mathcal{V}_{1}$ there exist $c_{1}, c_{2}>0$ such that

$$
\forall x \in \mathbb{R}_{-}^{n} \backslash \mathcal{V}_{2}, \quad\left(\left|x_{n}\right| \leq c_{1}\right) \Rightarrow\left(\tilde{f}(x) \geq c_{2}\right)
$$

With a cut-off $\chi_{1} \in \mathcal{C}_{0}^{\infty}(]-1,1[), \chi_{1}=1$ in a neighborhood of 0 , the 1 -form $\theta^{h}=\chi_{1}\left(\frac{x_{n}}{c_{1}}\right) e^{-\frac{\tilde{f}(x)}{h}} \tilde{\theta}^{h}$ satisfies

$$
\mathbf{t} \theta^{h}=0, \quad \mathbf{t} d_{\tilde{f}, h}^{*} \theta^{h}=\mathbf{i}_{\nabla \chi} u^{h}=r_{1}^{h}, \text { with }\left\|\theta^{h}\right\|_{\Lambda^{1} H^{2}}=\mathcal{O}\left(e^{-\frac{c_{3}}{h}}\right) .
$$

Hence the form $w^{h}=v^{h}-\theta^{h}$ belongs to the domain of $\Delta_{\tilde{f}}^{D T,(1)}$ and solves

$$
\left(\Delta_{\tilde{f}, h}^{(1)}-E_{1}(h)\right) w^{h}=r_{2}^{h},
$$


with $\left\|w^{h}\right\|_{\Lambda^{1} L^{2}}=1+\mathcal{O}\left(e^{-c_{3} / h}\right)$ and $\left\|r^{2}\right\|_{\Lambda^{1} L^{2}}=\mathcal{O}\left(e^{-c_{3} / h}\right)$. The spectral theorem then implies that there exists an eigenvalue $\lambda(h)$ of $\Delta_{\tilde{f}, h}^{(1)}$ such that

$$
\left|E_{1}(h)-\lambda(h)\right|=\mathcal{O}\left(e^{-c_{3} / h}\right) .
$$

The inclusion, $\sigma\left(\Delta_{\tilde{f}, h}^{(1)}\right) \backslash\{0\} \subset\left[C h^{6 / 5},+\infty\right)$, with $E_{1}(h)=\mathcal{O}\left(h^{3 / 2}\right)$, implies $\lambda(h)=0$.

\subsection{Accurate comparison with the WKB solution.}

We now compare the eigenvector associated with an exponentially small eigenvalue with its WKB approximation. We adapt the method presented in [He2, HelSj2] by following the same strategy as in Subsection 4.4. The $H^{1}$-estimates are done in two steps with $\varphi^{h}$ similar to $\frac{1}{2} f_{-}$and then with $\varphi^{h}$ similar to $\Phi$. Finally the elliptic regularity is used for the $\mathcal{C}^{\infty}$-estimates.

Proof of Proposition 4.2-d).

Let $u^{h} \in D\left(\Delta_{f, h}^{D, D T,(1)}\right)$ be an eigenvector associated with the first eigenvalue $E_{1}(h)$ of $\Delta_{f, h}^{D, D T,(1)}$ :

$$
\Delta_{f, h}^{D D T,(1)} u^{h}=E_{1}(h) u^{h},\left\|u^{h}\right\|=1 .
$$

According to Proposition 4.2-c), we know that $E_{1}(h)=\mathcal{O}\left(e^{-\frac{\varepsilon_{\rho}}{h}}\right)$, with $\varepsilon_{\rho}>0$, while the second eigenvalue of $\mathcal{D}_{f, h}^{D, D T,(1)}$ is larger than $h^{3 / 2}$.

By taking $\rho>0$ small enough, the WKB approximation $u_{1}^{w k b}$ presented in Subsection 4.2 satisfies

$$
\left\{\begin{array}{l}
\Delta_{f, h}^{(1)} u_{1}^{w k b}=\mathcal{O}\left(h^{\infty}\right) e^{-\frac{\Phi(x)}{h}} \text { in } \Omega_{U_{0}, \rho} \\
\left.\mathbf{t} u_{1}^{w k b}\right|_{\Gamma_{T D}}=0 \\
\left.\mathbf{t} d_{f, h}^{*} u_{1}^{w k b}\right|_{\Gamma_{T D}}=\mathcal{O}\left(h^{\infty}\right) e^{-\frac{\Phi(x)}{h}}
\end{array}\right.
$$

and there exists a $c>0$, such that for any $\rho^{\prime}>0$, we have

$$
\left\|u_{1}^{w k b}\right\|_{\Lambda^{1} L^{2}\left(\Omega_{U_{0}, \rho^{\prime}}\right)} \sim c h^{\frac{n+1}{4}} .
$$


The cut-off function $\chi \in \mathcal{C}^{\infty}\left(\overline{\Omega_{U_{0}, \rho}}\right)$ is supported in $\Omega_{U_{0}, \rho / 2} \cup \Gamma_{T D}$ and satisfies $\chi=1$ on $\Omega_{U_{0}, \rho^{\prime}}$, with $0<\rho^{\prime}<\rho / 2$. Later, we will take $\rho^{\prime}>0$ small enough, so that $\chi$ can be taken in the form

$$
\chi\left(x^{\prime}, x_{n}\right)=\chi_{1}\left(x^{\prime}\right) \chi_{n}\left(x_{n}\right) .
$$

By Lemma 2.8, the real constant factor $c(h)$ in the truncated WKB approximation $v_{1}^{w k b}=c(h) \chi u_{1}^{w k b}$ can be chosen so that

$$
\left\|v_{1}^{w k b}-u_{1}^{h}\right\|_{\Lambda^{1} H^{1}}=\mathcal{O}\left(h^{\infty}\right)
$$

and, due to the exponential decay of $u_{1}^{h}$ and $u_{1}^{w k b}$,

$$
\left\|\chi\left(u_{1}^{h}-c(h) u_{1}^{w k b}\right)\right\|_{\Lambda^{1} H^{1}}=\mathcal{O}\left(h^{\infty}\right) .
$$

We set

$$
w^{h}=\chi\left(u_{1}^{h}-c(h) u_{1}^{w k b}\right) .
$$

The 1-form $w^{h}$ satisfies in $\Omega_{U_{0}, \rho}$

$$
\begin{aligned}
\left(\Delta_{f, h}^{(1)}-E_{1}(h)\right) w^{h}= & \chi(x)\left(\Delta_{f, h}^{(1)}-E_{1}(h)\right)\left(u_{1}^{h}-c(h) u_{1}^{w k b}\right) \\
& +\left[\Delta_{f, h}^{(1)}, \chi\right]\left(u_{1}^{h}-c(h) u_{1}^{w k b}\right) \\
= & \tilde{r}_{0}^{h} e^{-\frac{\Phi(x)}{h}}+r_{0}^{h}
\end{aligned}
$$

where $\tilde{r}_{0}^{h}$ and $r_{0}^{h}$ satisfy, according to Proposition 4.2-b),

$$
\tilde{r}_{0}^{h}=\mathcal{O}\left(h^{\infty}\right), \quad \operatorname{supp} r_{0}^{h} \subset \operatorname{supp} \nabla \chi \quad \text { and } \quad r_{0}^{h}=\mathcal{O}\left(h^{-N_{0}}\right) e^{-\frac{\Phi(x)}{h}} .
$$

The last estimate can be done for any $\mathcal{C}^{k_{0}}$-norm, with $k_{0} \in \mathbb{N}$.

On the boundary $\partial \Omega_{U_{0}, \rho}=\Gamma_{T D} \cup \Gamma_{D}$, we have similarly

$$
\begin{gathered}
\left.\quad \mathbf{t} w^{h}\right|_{\Gamma_{T D}}=0,\left.\quad w^{h}\right|_{\Gamma_{D}}=0, \\
\text { and }\left.\quad \mathbf{t} d_{f, h}^{*} w^{h}\right|_{\Gamma_{T D}}=\tilde{r}_{1}^{h}\left(x^{\prime}\right) e^{-\frac{f(x)}{h}}+r_{1}^{h},
\end{gathered}
$$

with

$$
\tilde{r}_{1}^{h}=\mathcal{O}\left(h^{\infty}\right), \quad \operatorname{supp} r_{1}^{h} \subset \operatorname{supp} \nabla \chi \cap \Gamma_{T D} \quad \text { and } \quad r_{1}^{h}=\mathcal{O}\left(h^{-N_{0}}\right) e^{-\frac{f(x)}{h}} .
$$

With the different of choices for $\varphi^{h}$ given below, we will use the notation

$$
\tilde{w}^{h}=e^{\frac{\varphi^{h}}{h}} w^{h} \text {. }
$$


The 1-forms $w$ and $\tilde{w}$ belong to $\Lambda^{1} H^{2}\left(\Omega_{U_{0}, \rho}\right)$ and their supports do not meet $\Gamma_{D}$. Hence the integration by part formula (2.12) can be use in addition to (4.11).

\section{Step 1 : Comparison along $\Gamma_{T D}$.}

Like in the proof of Proposition 4.2-b) presented in Subsection 4.4, we introduce the sets

$$
\begin{aligned}
& \Omega_{-}^{h}=\left\{x=\left(x^{\prime}, x_{n}\right) \in \Omega_{U_{0}, \rho} ; \quad f_{-}\left(x^{\prime}\right)<C h\right\}, \\
& \text { and } \quad \Omega_{+}^{h}=\left\{x=\left(x^{\prime}, x_{n}\right) \in \Omega_{U_{0}, \rho} ; \quad f_{-}\left(x^{\prime}\right)>C h\right\} .
\end{aligned}
$$

For any $N \in \mathbb{N}$, we take

$$
\varphi_{N}^{h}=\frac{1}{2} \varphi_{N,-}^{h}\left(x^{\prime}\right)
$$

with

$$
\begin{aligned}
& \varphi_{N,-}^{h}\left(x^{\prime}\right)=\min \left\{\varphi_{-}^{h}\left(x^{\prime}\right)+N h \log h^{-1}, \psi(x)\right\}, \\
& \varphi_{-}^{h}\left(x^{\prime}\right)= \begin{cases}f_{-}\left(x^{\prime}\right)-C h \log \frac{f_{-}\left(x^{\prime}\right)}{h}, & \text { if } f_{-}\left(x^{\prime}\right)>C h, \\
f_{-}\left(x^{\prime}\right)-C h \log C, & \text { if } f_{-}\left(x^{\prime}\right) \leq C h,\end{cases}
\end{aligned}
$$

and $\quad \psi\left(x^{\prime}\right)=\min \left\{\varphi_{-}^{h}\left(y^{\prime}\right)+(1-\varepsilon)\left|f_{-}\left(x^{\prime}\right)-f_{-}\left(y^{\prime}\right)\right|, y^{\prime} \in \operatorname{supp} \nabla \chi_{1}\right\}$.

We recall that the cut-off $\chi$ writes $\chi\left(x^{\prime}, x_{n}\right)=\chi_{1}\left(x^{\prime}\right) \chi_{n}\left(x_{n}\right)$. The constant $C \geq 1$ will be fixed at the end like in the proof of Proposition 4.2-b). The constants $\rho^{\prime} \in(0, \rho / 2)$ and $\varepsilon>0$ are chosen so that, for $h \in\left(0, h_{N, \rho^{\prime}, \varepsilon}\right)$,

$$
\varphi_{N,-}^{h}\left(x^{\prime}\right)=\varphi_{-}^{h}\left(x^{\prime}\right)+N h \log h^{-1} \text { in } \Omega_{U_{0}, \rho^{\prime}} .
$$

Note the inequalities

$$
\begin{aligned}
\varphi_{N}^{h}(x) & \leq \frac{1}{2} f_{-}(x)+N h \log h^{-1} \quad \text { in } \quad \Omega_{U_{0}, \rho} \\
\varphi_{N}^{h}(x) & \leq \frac{1}{2} f_{-}(x) \leq \Phi(x), \text { if } \quad x^{\prime} \in \operatorname{supp} \nabla \chi_{1}, \\
\text { and } \quad \varphi_{N}^{h}(x) & \leq \frac{1}{2} f_{-}(x)+N h \log h^{-1} \leq \Phi(x), \text { if } \quad x_{n} \in \operatorname{supp} \chi_{n}^{\prime} .
\end{aligned}
$$

In particular, we have for $h \in\left(0, h_{N, \rho^{\prime}, \varepsilon}\right)$

$$
\varphi_{N}^{h}(x) \leq \Phi(x), \text { for } x \in \operatorname{supp} \nabla \chi,
$$

which implies

$$
\left\|e^{\frac{\varphi_{N}^{h}}{h}} r_{0}^{h}\right\|_{\Lambda^{1} L^{2}}+\left\|e^{\frac{\varphi_{N}^{h}}{h}} r_{1}^{h}\right\|_{\Lambda^{0} L^{2}\left(\Gamma_{T D}\right)}=\mathcal{O}_{N}\left(h^{-N_{0}}\right) .
$$


We apply the integration by part formula (4.11), where the left-hand side is computed with $(2.12)$, and we obtain for the form $\tilde{w}^{h}=e^{\frac{\varphi_{N}^{h}}{h}} w^{h}$ :

$$
\begin{aligned}
&\left\|\tilde{r}_{0}^{h}+e^{\frac{\varphi_{N}^{h}(x)}{h}} r_{0}^{h}\right\|_{\Lambda^{1} L^{2}}\left\|\tilde{w}^{h}\right\|_{\Lambda^{1} L^{2}}+\left\|\tilde{r}_{1}^{h}+e^{\frac{\varphi_{N}^{h}(x)}{h}} r_{1}^{h}\right\|_{\Lambda^{0} L^{2}\left(\Gamma_{T D}\right)}\left\|\tilde{w}^{h}\right\|_{\Lambda^{1} L^{2}\left(\Gamma_{T D}\right)} \\
& \geq\left\|h d \tilde{w}^{h}\right\|_{\Lambda^{2} L^{2}}^{2}+\left\|h d^{*} \tilde{w}^{h}\right\|_{\Lambda^{0} L^{2}}^{2} \\
&+\left\langle\left|\nabla x_{n}\right|^{2} \tilde{w}^{h} \mid \tilde{w}^{h}\right\rangle_{\Lambda^{1} L^{2}}-h \int_{\Gamma_{T D}}\left\langle\tilde{w}^{h} \mid \tilde{w}^{h}\right\rangle_{\Lambda^{1} T_{\sigma}^{*} \Omega}\left(\frac{\partial x_{n}}{\partial n}\right)(\sigma) d \sigma \\
&+\frac{1}{4}\left\langle\left(\left|\nabla f_{-}\right|^{2}-\left|\nabla \varphi_{N,-}^{h}\right|^{2}-4 C_{1} h\right) 1_{\Omega_{+}^{h}}(x) \tilde{w}^{h} \mid \tilde{w}^{h}\right\rangle,
\end{aligned}
$$

where the constant $C_{1}>0$ is determined by $f$ and $\tilde{r}_{\ell}^{h}=\mathcal{O}\left(h^{\infty}\right)$ for $\ell=1,2$. In $\Omega_{-}^{h}$ the weight $e^{\frac{\varphi_{N}^{h}(x)}{h}}$ is bounded by $C_{2}(C) h^{-N}$ and this provides

$$
\left\|\tilde{w}^{h}\right\|_{\Lambda^{1} L^{2}\left(\Omega_{-}^{h}\right)} \leq C_{2}(C) h^{-N}\left\|w^{h}\right\|_{\Lambda^{1} L^{2}\left(\Omega_{-}^{h}\right)} \leq C_{3}(C, N),
$$

due to $\left\|w^{h}\right\|_{\Lambda^{1} H^{1}}=\mathcal{O}\left(h^{\infty}\right)$.

Hence we can add to both sides of the previous inequality the term $C_{1} h\left\|\tilde{w}^{h}\right\|_{\Lambda^{1} L^{2}\left(\Omega_{-}^{h}\right)}^{2}$, which is controlled in the left-hand side by a $(C, N)$-dependent constant. We obtain

$$
\begin{aligned}
& C_{3}(C, N)\left(h^{-N_{0}}\left\|\tilde{w}^{h}\right\|_{\Lambda^{1} H^{1}}+1\right) \geq\left\|h d \tilde{w}^{h}\right\|_{\Lambda^{2} L^{2}}^{2}+\left\|h d^{*} \tilde{w}^{h}\right\|_{\Lambda^{0} L^{2}}^{2} \\
& \quad+\left\langle\left|\nabla x_{n}\right|^{2} \tilde{w}^{h} \mid \tilde{w}^{h}\right\rangle_{\Lambda^{1} L^{2}}-h \int_{\Gamma_{T D}}\left\langle\tilde{w}^{h} \mid \tilde{w}^{h}\right\rangle_{\Lambda^{1} T_{\sigma}^{*} \Omega}\left(\frac{\partial x_{n}}{\partial n}\right)(\sigma) d \sigma \\
& \quad+\frac{1}{4}\left\langle\left(\left|\nabla f_{-}\right|^{2}-\left|\nabla \varphi_{N,-}^{h}\right|^{2}-4 C_{1} h\right) 1_{\Omega_{+}^{h}}(x) \tilde{w}^{h} \mid \tilde{w}^{h}\right\rangle+C_{1} h\left\langle 1_{\Omega_{-}^{h}}(x) \tilde{w}^{h} \mid \tilde{w}^{h}\right\rangle .
\end{aligned}
$$

In $\Omega_{+}^{h}$, the point $x$ fulfills almost surely one of the two possibilities :

- Either $\nabla \varphi_{N,-}^{h}=\nabla \psi$, and we get

$$
\frac{1}{4}\left(\left|\nabla f_{-}\right|^{2}-\left|\nabla \varphi_{N,-}^{h}\right|^{2}\right) \geq \frac{2 \varepsilon-\varepsilon^{2}}{4}\left|\nabla f_{-}\left(x^{\prime}\right)\right|^{2} \geq \delta_{\rho, \varepsilon}>0
$$

where the last lower bound is due to the fact that $\varphi_{N,-}(x)=\psi(x)$ cannot occur in a neighborhood of $x^{\prime}=0$ for $\epsilon>0$ small enough and $h \in\left(0, h_{N, \rho^{\prime}, \epsilon}\right)$ 
- or $\nabla \varphi_{N,-}^{h}=\nabla f_{-}\left(1-\frac{C h}{f_{-}\left(x^{\prime}\right)}\right)$, and we get like in the proof of Proposition $4.2-\mathrm{b})$

$$
\frac{1}{4}\left(\left|\nabla f_{-}\right|^{2}-\left|\nabla \varphi_{N,-}^{h}\right|^{2}\right) \geq \frac{C}{C_{4}} h
$$

Taking $C \geq \max \left(1,2 C_{1} C_{4}\right)$ and $h \in\left(0, h_{N, \rho^{\prime}, \epsilon}\right]$, with $h_{N, \rho^{\prime}, \epsilon}>0$ small enough, leads to

$$
\begin{aligned}
C_{3}(C, N)\left(h^{-N_{0}}\left\|\tilde{w}^{h}\right\|_{\Lambda^{1} H^{1}}+1\right) \geq & \left\|h d \tilde{w}^{h}\right\|_{\Lambda^{2} L^{2}}^{2}+\left\|h d^{*} \tilde{w}^{h}\right\|_{\Lambda^{0} L^{2}}^{2} \\
& +\left\langle\left|\nabla x_{n}\right|^{2} \tilde{w}^{h} \mid \tilde{w}^{h}\right\rangle_{\Lambda^{1} L^{2}} \\
& -h \int_{\Gamma_{T D}}\left\langle\tilde{w}^{h} \mid \tilde{w}^{h}\right\rangle_{\Lambda^{1} T_{\sigma}^{*} \Omega}\left(\frac{\partial x_{n}}{\partial n}\right)(\sigma) d \sigma \\
& +2 \delta(C) h\left\|\tilde{w}^{h}\right\|_{\Lambda^{1} L^{2}}^{2} .
\end{aligned}
$$

After treating the right-hand side like in the proof of Proposition 4.2-b)Step 1, we obtain, for a possibly larger $N_{0}$,

$$
\left\|\tilde{w}^{h}\right\|_{\Lambda^{1} H^{1}\left(\Omega_{U_{0}, \rho}\right)} \leq C_{4} h^{-N_{0}} .
$$

Our choice of $\left(\varepsilon, \rho^{\prime}\right)$ imply

$$
\forall x \in \Omega_{U_{0}, \rho^{\prime}}, \quad \varphi_{N}^{h} \geq f(x)+N h \log h^{-1}-C \log \frac{C_{5}}{h} .
$$

We have proved the existence of $N_{1}$ and $\rho_{0}^{\prime}$, such that, for any $N \in \mathbb{N}$ and $\rho^{\prime} \in\left(0, \rho_{0}^{\prime}\right]$, there exists $h_{N, \rho^{\prime}}>0$ and $C_{N, \rho^{\prime}}>0$, such that

$$
\left\|e^{\frac{f_{-}}{2 h}}\left(u_{1}^{h}-c(h) u_{1}^{w k b}\right)\right\|_{\Lambda^{1} H^{1}\left(\Omega_{U_{0}, \rho^{\prime}}\right)} \leq C_{N, \rho^{\prime}} h^{N-N_{1}}
$$

holds for any $h \in\left(0, h_{N, \rho^{\prime}}\right)$. This last estimate and $\left.\Phi\right|_{\Gamma_{T D}}=\left.f\right|_{\Gamma_{T D}}=\left.\frac{1}{2} f_{-}\right|_{\Gamma_{T D}}$ imply

$$
\left\|e^{\frac{\Phi}{h}}\left(u_{1}^{h}-c(h) u_{1}^{w k b}\right)\right\|_{\Lambda^{1} H^{1 / 2}\left(\Omega_{U_{0}, \rho^{\prime}} \cap \Gamma_{T D}\right)}=\mathcal{O}\left(h^{\infty}\right) .
$$

Step 2 : Comparison in the normal direction.

After replacing $\rho^{\prime}$ by $\rho$, Step 1 provides the estimate

$$
\left\|e^{\frac{f}{h}}\left(u_{1}^{h}-c(h) u_{1}^{w k b}\right)\right\|_{\Lambda^{1} H^{1}}=\mathcal{O}\left(h^{\infty}\right) .
$$


We work in $\Omega_{U_{0}, \rho}$ with the above estimate and $\rho^{\prime} \in(0, \rho / 2)$ will be taken again small enough.

In order to get the interior estimate with the weight $e^{\frac{\Phi}{h}}$, we modify the previous analysis like in the proof of Proposition 4.2-b). The sets $\Omega_{ \pm}^{h}$ are now given by

$$
\begin{aligned}
\Omega_{-}^{h} & =\left\{x=\left(x^{\prime}, x_{n}\right) \in \Omega_{U_{0}, \rho} ; 2\left|x_{n}\right|<C h\right\} \\
\text { and } \quad \Omega_{+}^{h} & =\left\{x=\left(x^{\prime}, x_{n}\right) \in \Omega_{U_{0}, \rho} ; 2\left|x_{n}\right|>C h\right\} .
\end{aligned}
$$

The function $\varphi_{N}^{h}, N \in \mathbb{N}$, is given by

$$
\begin{aligned}
& \varphi_{N}^{h}(x)=\min \left\{\varphi^{h}(x)+N h \log h^{-1}, \psi(x)\right\}, \\
& \text { with } \quad \varphi^{h}(x)=\frac{1}{2} \varphi_{+}^{h}\left(x_{n}\right)+\frac{1}{2} f_{-}\left(x^{\prime}\right), \\
& \varphi_{+}^{h}\left(x_{n}\right)= \begin{cases}2\left|x_{n}\right|-C h \log \frac{2\left|x_{n}\right|}{h}, & \text { if } 2\left|x_{n}\right|>C h, \\
2\left|x_{n}\right|-C h \log C, & \text { if } 2\left|x_{n}\right| \leq C h,\end{cases} \\
& \text { and } \quad \psi(x)=\min \left\{\varphi^{h}(y)+(1-\varepsilon) d_{A g}(x, y), y \in \operatorname{supp} \nabla \chi\right\} .
\end{aligned}
$$

We recall that the Agmon distance $d_{A g}(x, y)$ is the distance between $x$ and $y$ for the metric $|\nabla f|^{2} d x^{2}$ and $\left.\Phi(x)=d_{A g}\left(x, U_{0}\right)\right)$.

Again, the constant $C \geq 1$ will be fixed in the end like in the proof of Proposition 4.2-b), while the constants $\rho^{\prime} \in(0, \rho / 2)$ and $\varepsilon>0$ are chosen so that

$$
\varphi_{N}^{h}(x)=\varphi^{h}(x)+N h \log h^{-1} \text { in } \Omega_{U_{0}, \rho^{\prime}} .
$$

Now we have the inequalities

$$
\begin{aligned}
\varphi_{N}^{h}(x) & \leq \Phi(x)+N h \log h^{-1} \quad \text { in } \quad \Omega_{U_{0}, \rho} \\
\text { and } \quad & \varphi_{N}^{h}(x) \leq \Phi(x) \text { in } \operatorname{supp} \nabla \chi .
\end{aligned}
$$

Hence the estimate,

$$
\left\|e^{\frac{\varphi_{N}^{h}}{h}} r_{0}^{h}\right\|_{\Lambda^{1} L^{2}}+\left\|e^{\frac{\varphi_{N}^{h}}{h}} r_{1}^{h}\right\|_{\Lambda^{0} L^{2}\left(\Gamma_{T D}\right)}=\mathcal{O}\left(h^{-N_{0}}\right),
$$

is still valid.

The inequality (4.17) implies that the $L^{2}$-norm of the trace of $\tilde{w}^{h}$ on $\Gamma_{T D}$ is $\mathcal{O}\left(h^{\infty}\right)$ and provides

$$
\left\|\tilde{w}^{h}\right\|_{\Lambda^{1} L^{2}\left(\Omega_{-}^{h}\right)} \leq C_{2}(C) h^{-N}\left\|e^{\frac{f_{-}}{2 h}} w^{h}\right\|_{\Lambda^{1} L^{2}\left(\Omega_{-}^{h}\right)} \leq \frac{1}{2} C_{3}(C, N) .
$$


With these estimates, the integration by part formula (4.11) and (2.12) lead to

$$
\begin{aligned}
C_{3}(C, N) & \left(h^{-N_{0}}\left\|\tilde{w}^{h}\right\|_{\Lambda^{1} L^{2}}+1\right) \geq\left\|h d \tilde{w}^{h}\right\|_{\Lambda^{2} L^{2}}^{2}+\left\|h d^{*} \tilde{w}^{h}\right\|_{\Lambda^{0} L^{2}}^{2} \\
& +\left\langle\left(|\nabla f|^{2}-\left|\nabla \varphi_{N}^{h}\right|^{2}-C_{1} h\right) 1_{\Omega_{+}^{h}}(x) \tilde{w}^{h} \mid \tilde{w}^{h}\right\rangle+C_{1} h\left\|\tilde{w}^{h}\right\|_{\Lambda^{1} L^{2}\left(\Omega_{-}^{h}\right)}^{2} .
\end{aligned}
$$

Finally, for almost all $x \in \Omega_{U_{0}, \rho}$ we have :

either : $\nabla \varphi_{N}^{h}(x)=\nabla \psi(x)$

and

$$
|\nabla f|^{2}-\left|\nabla \varphi_{N}^{h}\right|^{2}=\left(2 \varepsilon-\varepsilon^{2}\right)|\nabla f(x)|^{2} \geq \delta_{\rho, \varepsilon}>0 ;
$$

or : $\nabla \varphi_{N}^{h}(x)=\nabla \varphi^{h}(x)$

and we get like in the proof of Proposition 4.2-b)

$$
|\nabla f|^{2}-\left|\nabla \varphi^{h}\right|^{2} \geq 1-\frac{1}{4}\left|\nabla \varphi_{+}^{h}\left(x_{n}\right)\right|^{2} \geq \frac{C h}{4\left|x_{n}\right|} .
$$

By assuming $\left|x_{n}\right| \leq 1$ and by taking $C \geq \max \left(8 C_{1}, 1\right)$, we get that, $\left\|e^{\frac{\varphi_{N}^{h}}{h}} w^{h}\right\|=\mathcal{O}\left(h^{-N_{0}}\right)$, for some $N_{0}>0$.

Like in Step 1, this leads to

$$
\left\|e^{\frac{\Phi}{h}}\left(u_{1}^{h}-c(h) u_{1}^{w k b}\right)\right\|_{\Lambda^{1} H^{1}\left(\Omega_{U_{0}, \rho^{\prime}}\right)}=\mathcal{O}\left(h^{\infty}\right),
$$

for $\rho^{\prime} \in(0, \rho / 2)$ small enough.

\section{Step 3 :}

The estimates in higher order Sobolev spaces is done like in the proof of Proposition 4.2-b) by a bootstrap argument after writing a boundary value problem for $\chi\left(u_{1}^{h}-c(h) u_{1}^{w k b}\right)$ in $\mathbb{R}_{-}^{n}$. 


\section{$5 \quad$ Saddle sets and main assumptions}

\subsection{Preliminaries}

Here we adapt to the case with boundary the method of selecting the proper critical points with index 1 that we used in [HKN]. Some definitions and intermediate quantities have to be modified in order to take into account the effect of the boundary. We recall that the intuition for getting the good labelling of local minima, which is useful even to state properly the assumptions and results, comes from the probabilistic approach. The local minima have to be labelled according to the decreasing order of exit times. We refer to [BoGayKl], [BEGK] and [FrWe] for details.

The existence of such a labelling is an assumption which is generically satisfied. After this, it is possible to construct accurately quasimodes leading, with the help of the Witten complex structure, to accurate asymptotic expansions of the low lying eigenvalues.

\subsection{Saddle sets.}

We recall that we work here on a compact connected oriented Riemannian manifold $\bar{\Omega}=\Omega \cup \partial \Omega$ with boundary and that the function $f$ satisfies Assumption 3.1. According to our preliminary results on the Witten Laplacian $\Delta_{f, h}^{D T}$ in Theorem 3.3, we introduce the following definition of generalized critical points with index 1.

\section{Definition 5.1.}

A point $U \in \bar{\Omega}$ will be called a generalized critical point of $f$ with index 1 if :

- either $U \in \Omega$ and $U$ is a critical point of $f$ with index 1 ,

- or $U \in \partial \Omega$ and $U$ is a local minimum of $\left.f\right|_{\partial \Omega}$, such that $\partial_{n} f(U)>0$ ( $n$ being the outgoing normal vector).

The set of generalized critical points with index 1 is denoted by $\mathcal{U}^{(1)}$. Meanwhile $\mathcal{U}^{(0)}$ denotes the set of local minima of $\left.f\right|_{\Omega}$. From now we will use the notation

$$
m_{p}=\# \mathcal{U}^{(p)}, \text { for } p=0,1
$$

instead of $m_{p}^{\bar{\Omega}}$.

Finally it is convenient to call $\mathcal{U}$ the union of all critical points of $f$ and $\left.f\right|_{\partial \Omega}$. 
The saddle set (or set of saddle points) will be defined in the same spirit as in $[\mathrm{HKN}]$ and chosen in $\mathcal{U}^{(1)}$. We need some notations.

\section{Definition 5.2.}

a) For any $E \subset \bar{\Omega}$, the set of connected components ${ }^{2}$ of $E$ is denoted by $\operatorname{Conn}(E)$.

b) For any $A, B \subset \bar{\Omega}, H(A, B)$ denotes the quantity

$$
\begin{aligned}
H(A, B)=\inf \{c \in(-\infty,+\infty), & \exists C \in \operatorname{Conn}\left(f^{-1}((-\infty, c])\right), \\
& C \cap A \neq \emptyset \text { and } C \cap B \neq \emptyset\} .
\end{aligned}
$$

This quantity $H(A, B)$ is the least height to be reached to go from $A$ to $B$. A simple result which was checked in $[\mathrm{HKN}]$ in a slightly more general framework is the

\section{Proposition 5.3.}

When $A$ and $B$ are closed nonempty subsets of $\bar{\Omega}, H(A, B)$ is a minimum:

$$
\exists C \in \operatorname{Conn}\left(f^{-1}((-\infty, H(A, B)])\right), C \cap A \neq \emptyset \text { and } C \cap B \neq \emptyset .
$$

We are now able to introduce the right notion of saddle set.

\section{Definition 5.4.}

Under Assumption 3.1, let $A$ and $B$ be two closed subsets of $\bar{\Omega}$. We say that $Z \subset \bar{\Omega}$ is a saddle set for $(A, B)$, if it is not empty and satisfies the following conditions :

(sp2) $\left\{C \in \operatorname{Conn}\left(f^{-1}((-\infty, H(A, B)]) \backslash Z\right), C \cap A \neq \emptyset, C \cap B \neq \emptyset\right\}=\emptyset$.

If we compare this definition to the definition of "strict" saddle set in [HKN], we note that we have dropped the conditions

$$
Z \cap A=\emptyset \text { and } Z \cap B=\emptyset .
$$

We will effectively use the notion with $\partial \Omega \subset B$ and so the saddle set can meet $B$.

In order to check that this definition is coherent, it is useful to recall a few remarks coming from the local analysis of a Morse function which satisfies Assumption 3.1.

\footnotetext{
${ }^{2}$ We remind that the connected components are non empty closed subsets relatively to the induced topology on $E$ and therefore, $\bar{\Omega}$ being assumed compact, they are compact if $E$ is a closed subset of $\bar{\Omega}$.
} 


\section{Local structure of the level sets of a Morse function}

In order to analyze the local situation near a point $x_{0}$ of $\bar{\Omega}$, let us introduce :

$$
A_{f}^{<}\left(x_{0}\right):=\left\{x \in \bar{\Omega} ; f(x)<f\left(x_{0}\right)\right\} \cap B_{x_{0}}
$$

where $B_{x_{0}}$ is a ball centered at $x_{0}$. Similarly, we can introduce

$$
A_{f}^{\leq}\left(x_{0}\right):=\left\{x \in \bar{\Omega} ; f(x) \leq f\left(x_{0}\right)\right\} \cap B_{x_{0}} .
$$

\section{Interior points :}

First we observe that, near a non critical point $x_{0} \in \Omega$ of $f$, one can find $B_{x_{0}}$ and a set of local coordinates such that

$$
A_{f}^{<}\left(x_{0}\right)=\left\{y_{1}<0\right\} \cap B_{x_{0}} .
$$

Secondly, if $x_{0}$ is a critical point of index $p$, then there exists a ball $B_{x_{0}}$ around $x_{0}$ and a set of local coordinates centered at $x_{0}$ such that

$$
A_{f}^{<}\left(x_{0}\right)=\left\{-\sum_{\ell=1}^{p} y_{\ell}^{2}+\sum_{\ell=p+1}^{n} y_{\ell}^{2}<0\right\} \cap B_{x_{0}},
$$

and

$$
A_{f}^{\leq}\left(x_{0}\right)=\left\{-\sum_{\ell=1}^{p} y_{\ell}^{2}+\sum_{\ell=p+1}^{n} y_{\ell}^{2} \leq 0\right\} \cap B_{x_{0}} .
$$

We now observe that

1. When $p=0$ (local minimum), $A_{f}^{<}\left(x_{0}\right)$ is empty and $A_{f}^{\leq}\left(x_{0}\right)$ is reduced to $\left\{x_{0}\right\}$.

2. When $p=1, A_{f}^{<}\left(x_{0}\right)$ has two connected components and $x_{0}$ belongs to the closure of each of the two components. This property is crucial in the discussion of ( $\mathrm{sp} 2)$.

3. When $p \geq 2, A_{f}^{<}\left(x_{0}\right)$ is (arcwise) connected.

\section{Points on the boundary :}

If $x_{0}$ belongs to $\partial \Omega$, Assumption 3.1 leads to two cases :

First case.

If $x_{0}$ is not a critical point of $\left.f\right|_{\partial \Omega}$, then the hypersurfaces $\left\{f=f\left(x_{0}\right)\right\}$ and 
$\partial \Omega$ intersect transversally in a neighborhood of $x_{0}$. Hence there is a ball $B_{x_{0}}$ around $x_{0}$ and a set of local coordinates such that

$$
A_{f}^{<}\left(x_{0}\right)=\left\{y_{1}<0, y_{n} \leq 0\right\} \cap B_{x_{0}},
$$

and

$$
A_{f}^{\leq}\left(x_{0}\right)=\left\{y_{1} \leq 0, y_{n} \leq 0\right\} \cap B_{x_{0}},
$$

with $\Omega \cap B_{x_{0}}=\left\{y_{n}<0\right\} \cap B_{x_{0}}$.

\section{Second case.}

If $x_{0}$ is a critical point of $\left.f\right|_{\partial \Omega}$ with index $p-1$ and with $\pm \partial_{n} f\left(x_{0}\right)>0$, there are local coordinates $\left(y_{1}, \ldots, y_{n-1}, y_{n}\right)$, constructed from the relations $(3.22)-(3.25)$, such that $\left(y_{1}, \ldots, y_{n-1}\right)$ are Morse coordinates for $\left.f\right|_{\partial \Omega}$ and such that

$$
A_{f}^{<}\left(x_{0}\right)=\left\{ \pm y_{n}-\sum_{i=1}^{p-1} y_{i}^{2}+\sum_{i=p}^{n-1} y_{i}^{2}<0, y_{n} \leq 0\right\} \cap B_{x_{0}}
$$

and

$$
A_{f}^{\leq}\left(x_{0}\right)=\left\{ \pm y_{n}-\sum_{i=1}^{p-1} y_{i}^{2}+\sum_{i=p}^{n-1} y_{i}^{2} \leq 0, y_{n} \leq 0\right\} \cap B_{x_{0}} .
$$

These local models permit to see that

1. If $x_{0}$ is a local minimum of $\left.f\right|_{\partial \Omega}$ such that $\partial_{n} f\left(x_{0}\right)<0$, then $A_{f}^{<}\left(x_{0}\right)=\emptyset$ and $A_{f}^{\leq}\left(x_{0}\right)=\left\{x_{0}\right\}$.

2. If $x_{0}$ is a local minimum of $\left.f\right|_{\partial \Omega}$ such that $\partial_{n} f\left(x_{0}\right)>0$, then $A_{f}^{<}\left(x_{0}\right) \cap \partial \Omega=\emptyset$ and $A_{f}^{\leq}\left(x_{0}\right) \cap \partial \Omega=\left\{x_{0}\right\}$.

3. In all other cases, $A_{f}^{<}\left(x_{0}\right)$ admits one or two connected components with a non empty intersection with $\partial \Omega$ (two components if $p=2$ and $\partial_{n} f\left(x_{0}\right)<0$ and one in all other cases).

\section{Proposition 5.5.}

If $A$ and $B_{1}$ are disjoint non empty subsets of the set of the local minima of $f$ in $\Omega$, then the pair $(A, B)$, with $B=B_{1} \cup \partial \Omega$, admits a saddle set.

Proof.

We have to prove that a set $C$, belonging to $\operatorname{Conn}\left(f^{-1}((-\infty, H(A, B)])\right)$ 
and satisfying $C \cap A \neq \emptyset, C \cap B \neq \emptyset$ contains an element $z \in \mathcal{U}^{(1)}$ such that $f(z)=H(A, B)$. Then it suffices to take $Z=\mathcal{U}^{(1)} \cap f^{-1}((-\infty, H(A, B)])$. Let $C$ be a compact connected component of $f^{-1}((-\infty, H(A, B)])$ in $\bar{\Omega}$. Since $f$ is a Morse function, there are two possibilities, resulting from the previous local analysis of $f$ and of the connectedness of $C$ :

- Either it is reduced to one point which is a local minimum of $f$ in $\Omega$,

- or it is the closure of a finite union of bounded connected components $\Omega_{i}$ of $f^{-1}((-\infty, H(A, B)))$. Note that the $\Omega_{i}$ are open subsets of $\bar{\Omega}$.

The first case cannot occur. Indeed $C \cap A \neq \emptyset$ and $C \cap B \neq \emptyset$ would imply that the point $x_{C}$ (such that $\left.C=\left\{x_{C}\right\}\right)$ is a local minimum $\left(x_{C} \in A \subset \Omega\right.$ ) and belongs to $\partial \Omega\left(x_{C} \in B \backslash B_{1} \subset \partial \Omega\right)$.

Hence, we are in the second case and we have

$$
C=\cup_{i=1}^{N} \overline{\Omega_{i}},
$$

where $\Omega_{1}, \ldots, \Omega_{N}$ are bounded connected components of $f^{-1}((-\infty, H(A, B)))$. Every $x \in A \cap C$ (resp. $x \in B \cap C$ ) belongs to some $\Omega_{i}$. The $\Omega_{i}$ are labelled such that, for all $i \in\{1, \ldots, M\}, A \cap \Omega_{i} \neq \emptyset$, and for all $i \in\{M+1, \ldots, N\}$, $A \cap \Omega_{i}=\emptyset$. We have

$$
A \cap C \subset \cup_{i=1}^{M} \Omega_{i} \quad \text { and } \quad B_{1} \cap C \subset \bigcup_{i=M+1}^{N} \Omega_{i} .
$$

There are several cases :

If $C \subset \cup_{i=1}^{M} \overline{\Omega_{i}}$, then $C \cap B_{1}=\emptyset$ and $C \cap B \neq \emptyset$ imply that there exist $i \in\{1, \ldots, M\}$ and $x_{0} \in \bar{\Omega}$ such that $x_{0} \in \overline{\Omega_{i}} \cap \partial \Omega$. This implies first $f\left(x_{0}\right)=H(A, B)$ and the local description of $A_{f}^{<}\left(x_{0}\right)$ implies $x_{0} \in \mathcal{U}^{(1)} \cap \partial \Omega$. If $C \not \subset \cup_{i=1}^{M} \overline{\Omega_{i}}$, then $C \cap \cup_{i=M+1}^{N} \overline{\Omega_{i}} \neq \emptyset$. Since $C$ is connected, we have

$$
C \cap \overline{\left(\bigcup_{i=1}^{M} \Omega_{i}\right)} \cap \overline{\left(\bigcup_{j=M+1}^{N} \Omega_{j}\right)} \neq \emptyset .
$$

Therefore, there exist $i \leq M$ and $j \geq M+1$ such that $C \cap \overline{\Omega_{i}} \cap \overline{\Omega_{j}} \neq \emptyset$. Assume $x_{0} \in C \cap \overline{\Omega_{i}} \cap \overline{\Omega_{j}}$ and note that $i \neq j$ implies $f\left(x_{0}\right)=H(A, B)$. The local description of $A_{f}^{\leq}\left(x_{0}\right)$ says that $x_{0} \in \partial \Omega$ is possible only if it is a critical point of $\left.f\right|_{\partial \Omega}$ with index 1 . But again this cannot occur because $\Omega_{i} \cap A_{f}^{<}\left(x_{0}\right)$ would contain a point $x_{1} \in \partial \Omega$. Hence $x_{0} \in \Omega$. The local description of $A_{f}^{<}\left(x_{0}\right)$ shows that $x_{0}$ has to belong to $\mathcal{U}^{(1)}$. 


\section{On the uniqueness of the saddle set}

Like in the boundaryless case studied in $[\mathrm{HKN}]$, it is not possible to give a satisfactory definition of a unique saddle set and we introduce a new definition which explicitly specifies this case.

Definition 5.6.

Let $A, B$ be closed nonempty disjoint subsets of $\bar{\Omega}$. The point $z \in \mathcal{U}^{(1)}$ is said to be a unique (one point)-saddle set ${ }^{3}$ for the pair $(A, B)$ if

$$
\left(\cap_{C \in \mathcal{C}(A, B)} C\right) \cap\left[\mathcal{U}^{(1)} \cap f^{-1}(H(A, B))\right]=\{z\},
$$

where $\mathcal{C}(A, B)$ denotes the set of closed connected sets $C \subset f^{-1}((-\infty, H(A, B)])$, such that $C \cap A \neq \emptyset$ and $C \cap B \neq \emptyset$.

\subsection{Main assumption, notations and first consequences.}

We now give the main assumption like in [HKN] and inspired by [BEGK], which ensures that each exponentially small eigenvalue of $\Delta_{f, h}^{(0)}$ is simple, with a different asymptotic behavior.

We set here

$$
\mathcal{C}_{0}=\partial \Omega
$$

\section{Assumption 5.7.}

Under Assumption 3.1, there exists a labelling of the set of the local minima of $f \mathcal{U}^{(0)}=\left\{U_{1}^{(0)}, \ldots, U_{m_{0}}^{(0)}\right\}$ such that, by setting

$$
\mathcal{C}_{k}=\left\{U_{k}^{(0)}, \ldots, U_{1}^{(0)}\right\} \cup \mathcal{C}_{0}
$$

we have :

i) For all $k \in\left\{1, \ldots, m_{0}\right\}, U_{k}^{(0)}$ is the unique minimizer of

$$
H\left(U, \mathcal{C}_{k} \backslash\{U\}\right)-f(U), \quad U \in \mathcal{C}_{k} \backslash \mathcal{C}_{0}
$$

ii) For all $k \in\left\{1, \ldots, m_{0}\right\}$, the pair $\left(\left\{U_{k}^{(0)}\right\}, \mathcal{C}_{k-1}\right)$ admits a unique (one point)-saddle set $\left\{z_{k}^{*}\right\}$.

\footnotetext{
${ }^{3}$ or more shortly, a unique saddle point,
} 


\section{Remark 5.8.}

Like in [HKN], it is possible to check that this hypothesis is generically satisfied. More precisely, it is satisfied if all the critical values of $f$ are distinct and all the quantities $f\left(U^{(1)}\right)-f\left(U^{(0)}\right)$, with $U^{(1)} \in \mathcal{U}^{(1)}$ and $U^{(0)} \in \mathcal{U}^{(0)}$ are distinct. We refer the reader to [HKN].

By its definition, the point $z_{k}^{*}$ is a generalized critical point with index 1 , $z_{k}^{*} \in \mathcal{U}^{(1)}$.

Definition 5.9. (The map j)

If the generalized critical points of index 1 are numbered $U_{j}^{(1)}, j=1, \ldots, m_{1}$, we define the application $k \rightarrow j(k)$ on $\left\{1, \ldots, m_{0}\right\}$ by :

$$
U_{j(k)}^{(1)}=z_{k}^{*} .
$$

\section{Definition 5.10.}

Under Assumption 5.7 and for $k \in\left\{1, \ldots, m_{0}\right\}$, we denote by $E_{k}$ the connected component of $U_{k}^{(0)}$ in

$$
f^{-1}\left(\left(-\infty, f\left(U_{j(k)}^{(1)}\right)\right]\right) \backslash\left\{U_{j(k)}^{(1)}\right\} .
$$

\section{Proposition 5.11.}

Under Assumption 5.7, the following properties are satisfied:

a) The sequence $\left(f\left(U_{j(k)}^{(1)}\right)-f\left(U_{k}^{(0)}\right)\right)_{k \in\left\{1, \ldots, m_{0}\right\}}$ is strictly decreasing.

b) The set $E_{k}$ is a relatively compact subset of $f^{-1}\left(\left(-\infty, f\left(U_{j(k)}^{(1)}\right)\right]\right)$ and $\overline{E_{k}}=E_{k} \cup\left\{U_{j(k)}^{(1)}\right\}$ while $\overline{E_{k}} \cap \partial \Omega \subset\left\{U_{j(k)}^{(1)}\right\}$.

c) For any $(k, j) \in\left\{1, \ldots, m_{0}\right\} \times\left\{1, \ldots, m_{1}\right\}$, the relation $U_{j}^{(1)} \in E_{k}$ implies

$$
\text { either }\left(j=j\left(k^{\prime}\right) \text { for some } \quad k^{\prime}>k\right) \text { or } j \notin j\left(\left\{1, \ldots, m_{0}\right\}\right) \text {. }
$$

d) For any $k \neq k^{\prime} \in\left\{1, \ldots, m_{0}\right\}$, the relation $U_{k^{\prime}}^{(0)} \in E_{k}$ implies

$$
\left(k^{\prime}>k \quad \text { and } \quad f\left(U_{k^{\prime}}^{(0)}\right)>f\left(U_{k}^{(0)}\right)\right) .
$$

e) The application $j:\left\{1, \ldots, m_{0}\right\} \rightarrow\left\{1, \ldots, m_{1}\right\}$ is injective. 
Proof.

a) The condition i) of Assumption 5.7 gives

$$
\begin{aligned}
f\left(U_{j(k)}^{(1)}\right)-f\left(U_{k}^{(0)}\right) & =H\left(U_{k}^{(0)}, \mathcal{C}_{k} \backslash\left\{U_{k}^{(0)}\right\}\right)-f\left(U_{k}^{(0)}\right) \\
& <H\left(U_{k-1}^{(0)}, \mathcal{C}_{k} \backslash\left\{U_{k-1}^{(0)}\right\}\right)-f\left(U_{k-1}^{(0)}\right) \\
& \leq H\left(U_{k-1}^{(0)}, \mathcal{C}_{k-1} \backslash\left\{U_{k-1}^{(0)}\right\}\right)-f\left(U_{k-1}^{(0)}\right) \\
& =f\left(U_{j(k-1)}^{(1)}\right)-f\left(U_{k-1}^{(0)}\right) .
\end{aligned}
$$

b) It suffices to consider the local description of $A_{f}^{\leq}\left(U_{j(k)}^{(1)}\right)$ and $A_{f}^{<}\left(U_{j(k)}^{(1)}\right)$ in the two cases $U_{j(k)}^{(1)} \in \Omega$ and $U_{j(k)}^{(1)} \in \partial \Omega$. The last statement comes from $\mathcal{C}_{0}=\partial \Omega$ and the assumed uniqueness of a saddle point between $U_{k}^{(0)}$ and $\mathcal{C}_{k-1} \supset \mathcal{C}_{0}$.

c) Assume $U_{j\left(k^{\prime}\right)}^{(1)} \in E_{k}$.

Since $U_{j(k)}^{(1)} \notin E_{k}$, one has $k \neq k^{\prime}$. Moreover the inequality $f\left(U_{j\left(k^{\prime}\right)}^{(1)}\right) \leq$ $f\left(U_{j(k)}^{(1)}\right)$ implies that the connected component of $f^{-1}\left(\left(-\infty, f\left(U_{j\left(k^{\prime}\right)}^{(1)}\right)\right]\right)$, which contains $U_{j\left(k^{\prime}\right)}^{(1)}$ is contained in $\overline{E_{k}}$. Hence $E_{k}$ contains $U_{k}^{(0)}$ and $U_{k^{\prime}}^{(0)}$. Finally $E_{k}$ is modified into a closed connected set $\widehat{E}_{k}$ lying in $f^{-1}\left(\left(-\infty, f\left(U_{j(k)}^{(1)}\right)\right]\right) \backslash$ $\left\{U_{j(k)}^{(1)}\right\}$ in the following way. Take the coordinates $\left(x_{1}, \ldots, x_{n}\right)$ around $U_{j(k)}^{(1)}$ which are Morse coordinates if $U_{j(k)}^{(1)} \in \Omega$ and such that $f(x)-f\left(U_{j(k)}^{(1)}\right)=$ $x_{n}+\sum_{j=1}^{n-1} x_{j}^{2}$, if $U_{j(k)}^{(1)} \in \partial \Omega$. Consider, for $\rho>0$ small enough, $E_{k, \rho}:=$ $E_{k} \cap\{|x| \leq \rho\}$ and its radial projection on $E_{k, \rho}^{r e d}:=E_{k} \cap\{|x|=\rho\}$. Then $\widehat{E}_{k, \rho}:=\left(E_{k} \backslash E_{k, \rho}\right) \cup E_{k, \rho}^{r e d}$ is closed and can be considered as the image of $E_{k}$ by a continuous application. Hence it is connected. We have found a closed connected set $\widehat{E}_{k, \rho} \in \bar{\Omega}$ lying in $E_{k} \subset f^{-1}\left(\left(-\infty, f\left(U_{j(k)}^{(1)}\right)\right]\right)$, which contains $U_{k}^{(0)}$ and $U_{k^{\prime}}^{(0)}$, for $k^{\prime} \neq k$, and does not contain $U_{j(k)}^{(1)}$. Therefore one cannot have $k \leq k^{\prime}$, because this would contradict the assumption that $U_{j(k)}^{(1)}$ is the unique saddle point between $U_{k}^{(0)}$ and $\mathcal{C}_{k-1}$ (Assumption 5.7-ii) and Definition 5.6). Indeed the existence of another saddle point is obtained by using Proposition 5.5 by slightly increasing the value of $f\left(U_{j(k)}^{(1)}\right)$. Hence, the only possibility is $k^{\prime}>k$.

d) Assume $U_{k^{\prime}}^{(0)} \in E_{k}$ with $k \neq k^{\prime}$. By the same argument as for c), one then takes a closed connected set $C_{k, k^{\prime}} \subset E_{k} \subset f^{-1}\left(\left(-\infty, f\left(U_{j(k)}^{(1)}\right)\right]\right)$ such 
that $U_{k}^{(0)}, U_{k^{\prime}}^{(0)} \in C_{k, k^{\prime}}$ and $U_{j(k)}^{(1)} \notin C_{k, k^{\prime}}$. This implies $k^{\prime}>k$.

Assume now by contradiction that

$$
\left\{k^{\prime}>k, U_{k^{\prime}}^{(0)} \in E_{k} \text { and } f\left(U_{k^{\prime}}^{(0)}\right) \leq f\left(U_{k}^{(0)}\right)\right\} \neq \emptyset,
$$

and let $k_{0}$ be its smallest element.

We deduce from the existence of $C_{k, k_{0}}$ as a closed connected subset of $E_{k} \subset f^{-1}\left(\left(-\infty, f\left(U_{j(k)}^{(1)}\right)\right]\right)$ containing $U_{k}^{(0)}$ and $U_{k_{0}}^{(0)}$, the inequality

$$
f\left(U_{j\left(k_{0}\right)}^{(1)}\right)=H\left(U_{k_{0}}^{(0)}, \mathcal{C}_{k_{0}-1}\right) \leq f\left(U_{j(k)}^{(1)}\right) .
$$

Since the connected component $C$ of $U_{j\left(k_{0}\right)}^{(1)}$ in $f^{-1}\left(\left(-\infty, f\left(U_{j\left(k_{0}\right)}^{(1)}\right)\right]\right)$ contains $U_{k_{0}}^{(0)}$ and a point in $\mathcal{C}_{k_{0}-1}$, it is contained in $\overline{E_{k}}$ and $\overline{E_{k}}$ contains a point of $\mathcal{C}_{k_{0}-1}$. As a consequence of b), this point cannot belong to $\mathcal{C}_{0}$.

Hence there exists $k_{1}<k_{0}$ such that $U_{k_{1}}^{(0)} \in C \subset E_{k}$. Finally, the condition i) of Assumption 5.7 for $k_{0}$ gives

$$
\begin{aligned}
f\left(U_{j\left(k_{0}\right)}^{(1)}\right)-f\left(U_{k_{0}}^{(0)}\right) & =H\left(U_{k_{0}}^{(0)}, \mathcal{C}_{k_{0}-1}\right)-f\left(U_{k_{0}}^{(0)}\right) \\
& <H\left(U_{k_{1}}^{(0)}, \mathcal{C}_{k_{0}} \backslash\left\{U_{k_{1}}^{(0)}\right\}\right)-f\left(U_{k_{1}}^{(0)}\right) \\
& \leq f\left(U_{j\left(k_{0}\right)}^{(1)}\right)-f\left(U_{k_{1}}^{(0)}\right) .
\end{aligned}
$$

For the last inequality we used the existence of a connected set $C$ containing $U_{k_{1}}^{(0)}$ and the point $U_{k_{0}}^{(0)} \in \mathcal{C}_{k_{0}} \backslash\left\{U_{k_{1}}^{(0)}\right\}$ such that $f(C) \in\left(-\infty, f\left(U_{j(k)}^{(1)}\right)\right]$, with the definition of $H\left(U_{k_{1}}^{(0)}, \mathcal{C}_{k_{0}} \backslash\left\{U_{k_{1}}^{(0)}\right\}\right)$.

Hence we obtain

$$
f\left(U_{k_{1}}^{(0)}\right)<f\left(U_{k_{0}}^{(0)}\right) \leq f\left(U_{k}^{(0)}\right),
$$

with $k_{1}<k_{0}$ and $U_{k_{1}}^{(0)} \in E_{k}$ in contradiction with the definition of $k_{0}$. Hence we have proved

$$
\forall k^{\prime}>k,\left(U_{k^{\prime}}^{(0)} \in E_{k}\right) \Rightarrow\left(f\left(U_{k^{\prime}}^{(0)}\right)>f\left(U_{k}^{(0)}\right)\right)
$$

e) Assume $j(k)=j\left(k^{\prime}\right)$. The point $U_{j(k)}^{(1)}=U_{j\left(k^{\prime}\right)}^{(1)} \in \mathcal{U}^{(1)}$ is the unique saddle point for $\left(U_{k}^{(0)}, \mathcal{C}_{k-1}\right)$ and for $\left(U_{k^{\prime}}^{(0)}, \mathcal{C}_{k^{\prime}-1}\right)$.

Then we have

$$
\begin{aligned}
& \text { either } E_{k}=E_{k^{\prime}}, \\
& \text { or } \quad \exists k_{1}<k^{\prime}, U_{k_{1}}^{(0)} \in E_{k} \quad \text { and } \exists k_{2}<k, U_{k_{2}}^{(0)} \in E_{k^{\prime}} .
\end{aligned}
$$


According to d), the first case implies

$$
k \leq k^{\prime} \text { and } \quad k^{\prime} \leq k \text {, }
$$

while the second case gives

$$
k \leq k_{1}<k^{\prime} \quad \text { and } \quad k^{\prime} \leq k_{2}<k .
$$

Hence only the first case is possible with $k^{\prime}=k$.

\section{Quasimodes.}

Like in the boundaryless case, we associate with every $U_{k}^{(0)}\left(k \in\left\{1, \ldots, m_{0}\right\}\right)$ a quasimode for $\Delta_{f, h}^{D T,(0)}$ which is approximately supported in $E_{k}$, while the

quasimodes for $\Delta_{f, h}^{D T,(1)}$ will be supported in the balls $B\left(U_{j}^{(1)}, 2 \varepsilon_{1}\right), j \in$ $\left\{1, \ldots, m_{1}\right\}$. A ball $B(U, \rho)$, with $U \in \bar{\Omega}$ and $\rho>0$, is a geodesic ball and the geodesic distance is denoted by $d_{\Omega}$. The parameter $\varepsilon_{1}>0$ is fixed so that :

- $d_{\Omega}\left(U, U^{\prime}\right) \geq 10 \varepsilon_{1}$ for $U, U^{\prime} \in \mathcal{U}, U \neq U^{\prime}$.

- For all $U \in \mathcal{U}$ and all $k \in\left\{1, \ldots, m_{0}\right\}, U \notin \overline{E_{k}}$ implies

$$
d_{\Omega}\left(U, E_{k}\right) \geq 10 \varepsilon_{1} .
$$

- The construction of the WKB-approximation of Subsection 4.6 is possible in the ball $B\left(U_{j}^{(1)}, 2 \varepsilon_{1}\right)$. If $U_{j}^{(1)}$ is a boundary point, this means the introduction of the coordinates $\left(x^{\prime}, x_{n}\right)$ and the existence of $\Phi$.

The parameter $\varepsilon_{1}>0$ will be kept fixed, while we need another parameter $\varepsilon \in\left(0, \varepsilon_{0}\right)$, which has to be modified at each step of the final induction.

According to Proposition 5.11-b), Assumption 5.7 implies that $\overline{E_{k}}$ intersects $\partial \Omega$ at most at one point :

$$
\overline{E_{k}} \cap \partial \Omega \subset\left\{U_{j(k)}^{(1)}\right\} .
$$

The construction presented in [HKN] has to be adapted when this intersection is not empty and we focus on those changes. 
For every $k \in\left\{1, \ldots, m_{0}\right\}$, we introduce the open set

$$
\Omega_{k}=\stackrel{\circ}{E_{k}} \cup\left(\underset{U \in \mathcal{U} \cap \partial E_{k}, U \neq U_{j(k)}^{(1)}}{\cup} B\left(U, 3 \varepsilon_{1}\right)\right),
$$

which satisfies

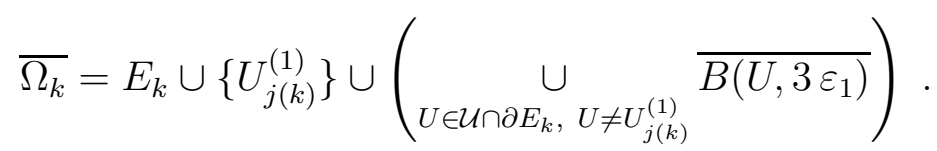

For $\varepsilon>0$, this set $\Omega_{k}$ is modified as

$$
\widetilde{\Omega}_{k}(\varepsilon, \delta)=\left\{x \in \Omega, d_{\Omega}\left(x, \overline{\Omega_{k}} \backslash B\left(U_{j(k)}^{(1)}, \varepsilon\right)\right)<\delta\right\} \cup B\left(U_{j(k)}^{(1)}, \varepsilon\right),
$$

with $\delta \in\left(0, \varepsilon_{\varepsilon}\right), \delta_{\varepsilon}>0$ small enough.

The cut-off function $\chi_{k, \varepsilon} \in \mathcal{C}_{0}^{\infty}(\Omega), 0 \leq \chi_{k, \varepsilon} \leq 1$ is chosen so that

$$
\operatorname{supp} \chi_{k, \varepsilon} \subset \widetilde{\Omega}_{k}\left(\varepsilon, \delta_{\varepsilon}\right) \text { and }\left.\chi_{k, \varepsilon}\right|_{\widetilde{\Omega}_{k}\left(\varepsilon, \delta_{\varepsilon} / 2\right) \backslash B\left(U_{j(k)}^{(1)}, \varepsilon\right)}=1 \text {. }
$$

Around $U_{j(k)}^{(1)}$, the cut-off function $\chi_{k, \varepsilon}$ is chosen (more accurately below) so that $U_{j(k)}^{(1)} \notin \operatorname{supp} \chi_{k, \varepsilon}$ and

$$
\forall x \in B\left(U_{j(k)}^{(1)}, \varepsilon\right),\left(\chi_{k, \varepsilon}(x) \neq 0 \text {, and } f(x)<f\left(U_{j(k)}^{(1)}\right)\right) \Rightarrow\left(x \in \stackrel{\circ}{E_{k}} \subset \Omega_{k}\right) .
$$

Like in $[\mathrm{HKN}]$ we deduce from Proposition 5.11 the following properties for $\chi_{k, \varepsilon}$.

\section{Proposition 6.1.}

By taking $\delta=\delta_{\varepsilon}$ with $\varepsilon \in\left(0, \varepsilon_{0}\right], 0<\varepsilon_{0} \leq \varepsilon_{1}$ small enough, the cut-off functions $\chi_{k, \varepsilon}\left(k \in\left\{1, \ldots, m_{0}\right\}\right)$ satisfy the following properties :

a) If $x$ belongs to $\operatorname{supp} \chi_{k, \varepsilon}$ and $f(x)<f\left(U_{j(k)}^{(1)}\right)$, then $x \in \stackrel{\circ}{E_{k}}$.

b) There exist $C>0$ and, for any $\varepsilon \in\left(0, \varepsilon_{0}\right]$, a constant $C_{\varepsilon}>0$, such that, for $x \in \operatorname{supp} \nabla \chi_{k, \varepsilon}$,

$$
\begin{aligned}
& \text { either } x \notin B\left(U_{j(k)}^{(1)}, \varepsilon\right) \quad \text { and } \quad f\left(U_{j(k)}^{(1)}\right)+C_{\varepsilon}^{-1} \leq f(x) \leq f\left(U_{j(k)}^{(1)}\right)+C \varepsilon, \\
& \text { or } \quad x \in B\left(U_{j(k)}^{(1)}, \varepsilon\right) \quad \text { and } \quad\left|f(x)-f\left(U_{j(k)}^{(1)}\right)\right| \leq C \varepsilon .
\end{aligned}
$$


c) For any $U \in \mathcal{U}, U \neq U_{j(k)}^{(1)}$, the distance $d_{\Omega}\left(U\right.$, $\left.\operatorname{supp} \nabla \chi_{k, \varepsilon}\right)$ is bounded from below by $3 \varepsilon_{1}>0$. If in addition $U \in \operatorname{supp} \chi_{k, \varepsilon}$, then $U \in E_{k}$.

d) If, for some $k^{\prime} \in\left\{1, \ldots, m_{0}\right\}, U_{k^{\prime}}^{(0)}$ belongs to $\operatorname{supp} \chi_{k, \varepsilon}$, then $k^{\prime} \geq k$ and

$$
f\left(U_{k^{\prime}}^{(0)}\right)>f\left(U_{k}^{(0)}\right), \quad f\left(U_{j\left(k^{\prime}\right)}^{(1)}\right) \leq f\left(U_{j(k)}^{1}\right), \quad \text { if } k \neq k^{\prime} .
$$

e) For any $j \in\left\{1, \ldots, m_{1}\right\}$, such that $U_{j}^{(1)} \in \operatorname{supp} \chi_{k, \varepsilon}$,

$$
\begin{aligned}
\text { either } & j \notin j\left(\left\{1, \ldots, m_{0}\right\}\right), \\
\text { or } & j=j\left(k^{\prime}\right), \text { for some } k^{\prime} \geq k \text { and } U_{k^{\prime}}^{(0)} \in \operatorname{supp} \chi_{k, \varepsilon} .
\end{aligned}
$$

The cut-off function $\chi_{k, \varepsilon}$ is used in the construction of quasi-modes for $\Delta_{f, h}^{D T,(0)}$. Like in the boundaryless case, the construction of quasi-modes for $\Delta_{f, h}^{D T,(1)}$ will rely on the approximation by the Dirichlet problem in small neighborhoods of $U_{j}^{(1)}, j \in\left\{1, \ldots, m_{1}\right\}$. For interior points $U_{j}^{(1)} \in \Omega$, this neighborhood is $B\left(U_{j}^{(1)}, 2 \varepsilon_{1}\right)$. For points $U_{j}^{(1)}$ in the boundary $\partial \Omega$, this Dirichlet realization is $\Delta_{f, h}^{D, D T,(1)}$, which was studied in Subsection 4.6 and associated with the neighborhood $\Omega_{U_{j}^{(1)}, \rho}$ with $\rho>0$ small enough. Once $\rho>0$ is fixed uniformly for all $U_{j}^{(1)} \in \partial \Omega$, the parameter $\varepsilon_{1}>0$ is reduced so that $B\left(U_{j}^{(1)}, 2 \varepsilon_{1}\right) \subset \Omega_{U_{0}, \rho}$ for all $U_{j}^{(1)} \in \partial \Omega$. For all $j \in\left\{1, \ldots, m_{1}\right\}, u_{j}$ denotes a normalized eigenvector associated with the first (exponentially small) eigenvalue of this Dirichlet realization. The cut-off function $\theta_{j} \in \mathcal{C}_{0}^{\infty}\left(B\left(U_{j}^{(1)}, 2 \varepsilon_{1}\right)\right)$ is taken such that $\theta_{j}=1$ on $B\left(U_{j}^{(1)}, \varepsilon_{1}\right)$.

Note that the function $\chi_{k, \varepsilon}$ depends on $\varepsilon \in\left(0, \varepsilon_{0}\right]$, while $\theta_{j}$ is kept fixed like $\varepsilon_{1}>0$.

\section{Definition 6.2.}

For any $k \in\left\{1, \ldots, m_{0}\right\}$, the $(\varepsilon, h)$-dependent function $\psi_{k}^{(0)}$ is defined by

$$
\psi_{k}^{(0)}(x)=\left\|\chi_{k, \varepsilon}(x) e^{-\left(f(x)-f\left(U_{k}^{(0)}\right)\right) / h}\right\|^{-1} \chi_{k, \varepsilon}(x) e^{-\left(f(x)-f\left(U_{k}^{(0)}\right)\right) / h} .
$$

For any $j \in\left\{1, \ldots, m_{1}\right\}$, the $h$-dependent 1 -form $\psi_{j}^{(1)}$ is defined by

$$
\psi_{j}^{(1)}(x)=\left(\left\|\theta_{j} u_{j}\right\|^{-1}\right) \theta_{j}(x) u_{j}(x) .
$$


For any $k \in\left\{1, \ldots, m_{0}\right\}$, we set

$$
\lambda_{k}^{a p p}(\varepsilon, h)=\left|\left\langle\psi_{j(k)}^{(1)} \mid d_{f, h}^{(0)} \psi_{k}^{(0)}\right\rangle\right|^{2} .
$$

\section{Remark 6.3.}

a) For the sake of conciseness, we omit the $(\varepsilon, h)$ - and $h$-dependence in the notations $\psi_{k}^{(0)}$ and $\psi_{j}^{(1)}$.

b) Note that, for boundary points $U_{j}^{(1)} \in \partial \Omega$, the quasimode $\psi_{j}^{(1)}$ only belongs to the form domain $\Lambda^{1} H_{0, T}^{1}(\Omega)$ of $\Delta_{f, h}^{D T,(1)}$. This brings no additional difficulty to what was done in [HKN] for the boundaryless case, because the preliminary approximation of the spectral subspace with quasimodes only requires the MinMax principle or Lemma 2.8.

We end this section by reviewing the quasimodal estimates which are derived from Propositions 5.11 and 6.1. We refer the reader to [HKN] for the details. The asymptotic expansion of $\left\langle\psi_{j(k)}^{(1)} \mid d_{f, h}^{(0)} \psi_{k}^{(0)}\right\rangle$ has also be done in $[\mathrm{HKN}]$ when $U_{j(k)}^{(1)} \in \Omega$ is an interior point. We will simply complete this analysis by establishing the asymptotic expansion of $\left\langle\psi_{j(k)}^{(1)} \mid d_{f, h}^{(0)} \psi_{k}^{(0)}\right\rangle$, when $U_{j(k)}^{(1)} \in \partial \Omega$.

Remind that the parameter $\varepsilon_{1}>0$ is fixed, while $\varepsilon_{0}$ and $\varepsilon \in\left(0, \varepsilon_{0}\right]$ will be adapted in the different steps of the proof. We shall denote by $\alpha$ a generic positive constant which is independent of $\varepsilon \in\left(0, \varepsilon_{0}\right]$.

From Proposition 5.11-d) and the good localization of $\nabla \chi_{k, \varepsilon}$, we deduce the following estimates for $\psi_{k}^{(0)}$.

\section{Proposition 6.4.}

The system of $(\varepsilon, h)$-dependent functions $\left(\psi_{k}^{(0)}\right)_{k \in\left\{1, \ldots, m_{0}\right\}}$ of Definition 6.2 is almost orthogonal with

$$
\left(\left\langle\psi_{k}^{(0)} \mid \psi_{k^{\prime}}^{(0)}\right\rangle\right)_{k, k^{\prime} \in\left\{1, \ldots, m_{0}\right\}}=\operatorname{Id}_{\mathbb{C}^{m_{0}}}+\mathcal{O}_{\varepsilon}\left(e^{-\alpha / h}\right),
$$

and there exists $\alpha>0$ and, for any $\varepsilon \in\left(0, \varepsilon_{0}\right], C(\varepsilon)$ and $h_{0}(\varepsilon)$ such that, for any $h \in\left(0, h_{0}(\varepsilon)\right]$,

$$
\left\langle\Delta_{f, h}^{(0)} \psi_{k}^{(0)} \mid \psi_{k}^{(0)}\right\rangle=\left\|d_{f, h}^{(0)} \psi_{k}^{(0)}\right\|^{2} \leq C(\varepsilon) e^{-2\left(f\left(U_{j(k)}^{(1)}\right)-f\left(U_{k}^{(0)}\right)-\alpha \varepsilon\right) / h} .
$$




\section{Corollary 6.5.}

There exists $\varepsilon_{0}>0$ and $\alpha>0$ such that, for any choice of $\varepsilon$ in $\left(0, \varepsilon_{0}\right]$ and for all $k \in\left\{1, \ldots, m_{0}\right\}$, the $(\varepsilon, h)$-dependent quasimodes $\psi_{k}^{(0)}$ satisfy the estimate

$$
\left\langle\Delta_{f, h}^{(0)} \psi_{k}^{(0)} \mid \psi_{k}^{(0)}\right\rangle \leq C_{\varepsilon} e^{-\alpha / h}
$$

The exponential decay of the first eigenvector $u_{j}$, associated with an exponentially small eigenvalue, of the Dirichlet realization of $\Delta_{f, h}^{(1)}$ around $U_{j}^{(1)}$, provides the next estimates for $\psi_{j}^{(1)}$. We refer the reader to [HKN] or [HelSj4] for $U_{j}^{(1)} \in \Omega$ and to Subsection 4.6 for $U_{j}^{(1)} \in \partial \Omega$.

\section{Proposition 6.6.}

The system of $h$-dependent 1-forms, $\left(\psi_{j}^{(1)}\right)_{j \in\left\{1, \ldots, m_{1}\right\}}$ given in Definition 6.2 is orthonormal and there exists $\alpha>0$ independent of $\varepsilon$ such that

$$
\mathcal{D}_{f, h}\left(\psi_{j}^{(1)}\right)=\mathcal{O}\left(e^{-\alpha / h}\right),
$$

for all $j \in\left\{1, \ldots, m_{1}\right\}$.

Before we state the next result, let us specify the choice of $\chi_{k, \varepsilon}$ in $B\left(U_{j(k)}^{(1)}, \varepsilon\right)$ in the case when $U_{j(k)}^{(1)} \in \partial \Omega$. We assume $\varepsilon \in\left(0, \varepsilon_{0}\right)$, with $0<\varepsilon_{0}<\frac{\varepsilon_{1}}{10}$. We use again the coordinate system $\left(x^{\prime}, x_{n}\right)$ introduced in Section 3 and Subsection 4.6 such that :

and

$$
x^{\prime}\left(U_{j(k)}^{(1)}\right)=0, \quad x_{n}\left(U_{j(k)}^{(1)}\right)=0, \quad \partial \Omega \cap B\left(U_{j(k)}^{(1)}, 2 \varepsilon_{1}\right) \subset\left\{x_{n}=0\right\}
$$

$$
f\left(x^{\prime}, x_{n}\right)=f\left(U_{j(k)}^{(1)}\right)+x_{n}+\frac{1}{2} f_{-}\left(x^{\prime}\right), \quad x_{n}<0 \text { in } \Omega \cap B\left(U_{j(k)}^{(1)}, 2 \varepsilon_{1}\right) .
$$

The function $\Phi(x)$ which equals the Agmon distance $d_{A g}\left(x, U_{j(k)}^{(1)}\right)$ is given by

$$
\Phi\left(x^{\prime}, x_{n}\right)=-x_{n}+\frac{1}{2} f_{-}\left(x^{\prime}\right) .
$$

The construction of the coordinate system $\left(x^{\prime}, x_{n}\right)$ which block diagonalizes the metric everywhere (see (3.26)) permits ${ }^{4}$, when $n>1$, to choose the

\footnotetext{
${ }^{4}$ The case $n=1$ is easier (no Laplace method has to be used) and we refer the reader to the appendix.
} 
boundary coordinates $x^{\prime}=\left(x_{1}, \ldots, x_{n-1}\right)$ such that $d x_{1} \wedge d x_{2} \ldots \wedge d x_{n-1} \wedge d x_{n}$ is the Riemannian volume form in $B\left(U_{j(k)}^{(1)}, 2 \varepsilon_{1}\right)$. This means

$$
\star\left(d x_{1} \wedge \ldots \wedge d x_{n-1}\right)=d x_{n}, \quad \star d x_{n}=(-1)^{n-1} d x_{1} \wedge \ldots \wedge d x_{n-1} .
$$

The cut-off function $\chi_{k, \varepsilon}$ fulfills the following conditions which are illustrated in Figure 1 :

i) The support of $\chi_{k, \varepsilon}$ does not meet $\partial \Omega$ (already stated).

ii) In a neighborhood

$$
\mathcal{V}=\left\{x \in B\left(U_{j(k)}^{(1)}, \varepsilon\right), \quad\left|x^{\prime}\right|<\nu_{\varepsilon}\right\}
$$

of the curve $\left\{x^{\prime}=0, x_{n}<0\right\}$, the function $\chi_{k, \varepsilon}$ only depends on $x_{n}$ : $\chi_{k, \varepsilon}(x)=\chi_{k, \varepsilon}\left(x_{n}\right)$, for $x \in \mathcal{V}$.

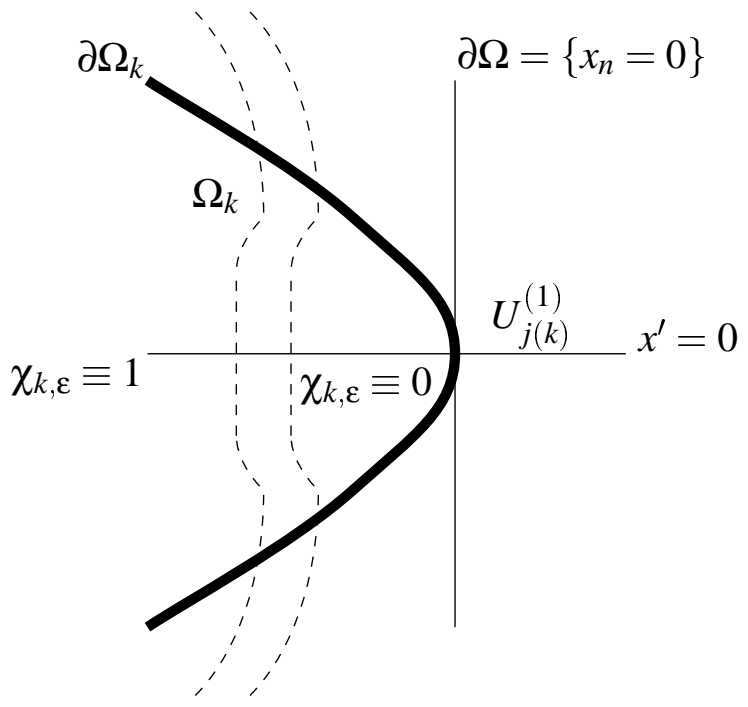

Figure 1: Case $U_{j(k)}^{(1)} \in \partial \Omega$. The support of $\nabla \chi_{k, \varepsilon}$ is localized between the dashed curve. The function $f$ is constant along $\partial \Omega_{k}$.

In Subsection 4.6, we found the WKB approximation $u_{1}^{w k b}$ of an eigenvector $u_{1}^{h}$, such that

$$
\begin{aligned}
& e^{\frac{\Phi(x)}{h}} u_{1}^{w k b}=-2 a_{0}(x) d x_{n}+h b^{1}(x, h), \\
& a_{0}(0)=1, b^{1}(x, h) \sim \sum_{\ell} h^{\ell} b_{\ell}(x)
\end{aligned}
$$


and

$$
\forall x \in B\left(U_{j(k)}^{(1)}, 2 \varepsilon_{1}\right), \quad e^{\frac{\Phi(x)}{h}}\left|\partial_{x}^{\alpha}\left(u_{1}^{h}(x)-u_{1}^{w k b}(x)\right)\right| \leq C_{\alpha, N} h^{N} .
$$

The normalized eigenvector that we take here is

$$
u_{j(k)}=\frac{(-1)^{n-1}}{\left\|u_{1}^{h}\right\|} u_{1}^{h} .
$$

Let us first compute accurately

$$
\left\|u_{1}^{h}\right\|=\left\|\theta_{j(k)} u_{1}^{h}\right\|+\mathcal{O}\left(h^{\infty}\right)=\left\|\theta_{j(k)} u_{1}^{w k b}\right\|+\mathcal{O}\left(h^{\infty}\right) .
$$

We have, denoting by $d x$ the Riemannian volume measure,

$$
\begin{aligned}
\left\|\theta_{j(k)} u_{1}^{w k b}\right\|^{2} & =\int 4 \theta_{j(k)}(x)^{2}\left\langle d x_{n} \mid d x_{n}\right\rangle a_{0}(x)^{2} e^{-\frac{2 \Phi(x)}{h}} d x \\
& =4 \int \theta_{j(k)}(x)^{2} a_{0}(x)^{2} e^{\frac{2 x_{n}}{h}} e^{-\frac{f_{-}\left(x^{\prime}\right)}{h}} d x_{1} \wedge \ldots \wedge d x_{n},
\end{aligned}
$$

where the integral is over $x_{n}<0$. The Laplace method, applied with the function $f_{-}=\left.2 f\right|_{\partial \Omega}-2 f\left(U_{j(k)}^{(1)}\right)$, gives

$$
\left\|\theta_{j(k)} u_{1}^{w k b}\right\|^{2}=2 h \frac{(\pi h)^{\frac{n-1}{2}}}{\left(\delta_{f, \partial \Omega}\left(U_{j(k)}^{(1)}\right)\right)^{1 / 2}}(1+\mathcal{O}(h))
$$

with

$$
\delta_{f, \partial \Omega}=\left|\operatorname{det}\left(\frac{1}{2}\left(\partial_{j k} f_{-}\right)_{j, k=1, \ldots, n-1}\right)\right| .
$$

Note that the Laplace method gives actually a full asymptotic expansion. After the normalization we get, for all $x \in B\left(U_{j(k)}^{(1)}\right)$,

$$
\psi_{j(k)}^{(1)}(x)=(-1)^{n} \sqrt{2 \pi} \frac{\left(\delta_{f, \partial \Omega}\left(U_{j(k)}^{(1)}\right)\right)^{1 / 4}}{(\pi h)^{\frac{n+1}{4}}}\left(b_{0 k}(x) d x_{n}+h b_{k}^{1}(x, h)\right) e^{-\frac{\Phi(x)}{h}},
$$

with $b_{0, k}(0)=1$ and $b_{k}^{1}(x, h) \sim \sum_{\ell=0}^{+\infty} h^{\ell} b_{k \ell}^{1}(x)$.

For the quasimode $\psi_{k}^{(0)}$, a direct Laplace method provides (see [HKN])

$$
\forall x \in \Omega, \quad \psi_{k}^{(0)}(x)=\frac{\left|\operatorname{det} \operatorname{Hess} f\left(U_{k}^{(0)}\right)\right|^{1 / 4}}{(\pi h)^{n / 4}} a_{k}(h) \chi_{k, \varepsilon}(x) e^{-\frac{f(x)-f\left(U_{k}^{(0)}\right)}{h}},
$$

with $a_{k}(h) \sim \sum_{\ell=0}^{\infty} h^{\ell} a_{k, \ell}$ and $a_{k, 0}=1$. 


\section{Proposition 6.7.}

There exist $\varepsilon_{0}$ and sequences $\left(c_{k, m}\right)_{m \in \mathbb{N}^{*}}$, such that the $(\varepsilon, h)$-dependent and $h$-dependent quasimodes $\psi_{k}^{(0)}$ and $\psi_{j}^{(1)}\left((k, j) \in\left\{1, \ldots, m_{0}\right\} \times\left\{1, \ldots, m_{1}\right\}\right.$ and $\left.\varepsilon \in\left(0, \varepsilon_{0}\right]\right)$ satisfy :

$$
\begin{gathered}
\left\langle\psi_{j}^{(1)} \mid d_{f, h}^{(0)} \psi_{k}^{(0)}\right\rangle=0 \quad \text { if } j \neq j(k), \\
\left\langle\psi_{j(k)}^{(1)} \mid d_{f, h}^{(0)} \psi_{k}^{(0)}\right\rangle=(-1)^{n-1} \frac{h^{1 / 2}}{\pi^{1 / 2}}\left|\widehat{\lambda}_{1}\left(U_{j(k)}^{(1)}\right)\right|^{1 / 2}\left|\frac{\operatorname{det}\left(\operatorname{Hess} f\left(U_{k}^{(0)}\right)\right)}{\operatorname{det}\left(\operatorname{Hess} f\left(U_{j(k)}^{(1)}\right)\right)}\right|^{1 / 4} \\
\times \exp -\frac{1}{h}\left(f\left(U_{j(k)}^{(1)}\right)-f\left(U_{k}^{(0)}\right)\right) \times\left[1+h c_{k}^{1}(h)\right], \\
\text { if } j=j(k) \text { and } U_{j(k)}^{(1)} \in \Omega,
\end{gathered}
$$

and

$$
\begin{gathered}
\left\langle\psi_{j(k)}^{(1)} \mid d_{f, h}^{(0)} \psi_{k}^{(0)}\right\rangle=(-1)^{n-1} \frac{\sqrt{2} h^{1 / 4}}{\pi^{1 / 4}}\left|\frac{\operatorname{det}\left(\operatorname{Hess} f\left(U_{k}^{(0)}\right)\right)}{\left.\delta_{f, \partial \Omega}\left(U_{j(k)}^{(1)}\right)\right)}\right|^{1 / 4} \\
\times \exp -\frac{1}{h}\left(f\left(U_{j(k)}^{(1)}\right)-f\left(U_{k}^{(0)}\right)\right) \times\left[1+h c_{k}^{1}(h)\right], \\
\text { if } j=j(k) \text { and } U_{j(k)}^{(1)} \in \partial \Omega,
\end{gathered}
$$

with $c_{k}^{1}(h) \sim \sum_{m=0}^{\infty} c_{k, m} h^{m}$.

\section{Remark 6.8.}

We recall that we were computing above in coordinates such that the Riemannian volume form is $d x_{1} \wedge \ldots \wedge d x_{n-1} \wedge d f\left(U_{j(k)}^{(1)}\right)$. The prefactor in the last formula of Proposition 6.7 can be expressed more intrinsically by observing that:

$$
\left|\delta_{f, \partial \Omega}\left(U_{j(k)}^{(1)}\right)\right|=\left|\nabla f\left(U_{j(k)}^{(1)}\right)\right|^{-2} \times\left|\operatorname{det}\left(\left.\operatorname{Hess} f\right|_{\partial \Omega}\left(U_{j(k)}^{(1)}\right)\right)\right| .
$$

Proof of Proposition 6.7.

The first statement for $j \neq j(k)$ is a consequence of our choice of $\varepsilon_{1}>0$ and $\chi_{k, \varepsilon}$ which gives according to Proposition 6.1-c) $\operatorname{supp} \psi_{j}^{(1)} \cap \operatorname{supp} \nabla \chi_{k, \varepsilon}=\emptyset$. We conclude with $d_{f, h}^{(0)} \psi_{k}^{(0)}=C_{\varepsilon, h}\left(d^{(0)} \chi_{k, \varepsilon}\right) e^{-f / h}$. 
The second case was completely treated in [HKN] for the boundaryless problem.

The last one, $j=j(k)$ with $U_{j(k)}^{(1)} \in \partial \Omega$, is adapted from the second one by using the specific approximations (6.6) and (6.5). With

$$
d_{f, h}^{(0)}\left(\chi_{k, \varepsilon} e^{-\frac{f(x)}{h}}\right)=e^{-\frac{f(x)}{h}} h d^{(0)} \chi_{k, \varepsilon},
$$

we obtain the existence, for any $\varepsilon>0$, of $\sigma_{\varepsilon}>0$ such that

$$
\begin{aligned}
\left\langle\psi_{j(k)}^{(1)} \mid d_{f, h}^{(0)} \psi_{k}^{(0)}\right\rangle & =h^{1-\frac{n}{4}} a_{k}(h) \frac{\left|\operatorname{det} \operatorname{Hess} f\left(U_{k}^{(0)}\right)\right|^{1 / 4}}{\pi^{n / 4}} \\
\times \int_{B\left(U_{j(k)}^{(1)}, \varepsilon\right)}\left\langle\psi_{j(k)}^{(1)} \mid d \chi_{k, \varepsilon}\right\rangle(x) e^{-\frac{\left(f(x)-f\left(U_{k}^{(0)}\right)\right)}{h}} d x & \\
& +\mathcal{O}_{\varepsilon}\left(e^{-\frac{f\left(U_{j(k)}^{(1)}\right)-f\left(U_{k}^{(0)}\right)+\sigma_{\varepsilon}}{h}}\right)
\end{aligned}
$$

with $a_{k}(h) \sim 1+\sum_{\ell=1}^{\infty} h^{\ell} a_{k, \ell}$.

The two additional conditions i) and ii) given above for the cutoff function $\chi_{k, \varepsilon}$ permit to reduce the integration domain to the neighborhood $\mathcal{V}$, introduced in (6.3) :

$$
\begin{aligned}
\left\langle\psi_{j(k)}^{(1)} \mid d_{f, h}^{(0)} \psi_{k}^{(0)}\right\rangle & =h^{1-\frac{n}{4}} a_{k}(h) \frac{\left|\operatorname{det} \operatorname{Hess} f\left(U_{k}^{(0)}\right)\right|^{1 / 4}}{\pi^{n / 4}} \\
\times \int_{\mathcal{V}}\left\langle\psi_{j(k)}^{(1)} \mid \chi_{k, \varepsilon}^{\prime} d x_{n}\right\rangle(x) e^{-\frac{\left(f(x)-f\left(U_{k}^{(0)}\right)\right)}{h}} d x & \\
& +\mathcal{O}_{\varepsilon}\left(e^{-\frac{f\left(U_{j(k)}^{(1)}\right)-f\left(U_{k}^{(0)}\right)+\sigma_{\varepsilon}}{h}}\right),
\end{aligned}
$$

for some $\sigma_{\varepsilon}>0$. 
Finally (6.5) and (6.2) lead to

$$
\begin{aligned}
& \left\langle\psi_{j(k)}^{(1)} \mid d_{f, h}^{(0)} \psi_{k}^{(0)}\right\rangle \\
& =h^{1-\frac{n}{2}-\frac{1}{4}} \frac{\sqrt{2 \pi}\left|\operatorname{det} \operatorname{Hess} f\left(U_{k}^{(0)}\right)\right|^{\frac{1}{4}}\left(\delta_{f, \partial_{\Omega}}\left(U_{j(k)}^{(1)}\right)\right)^{1 / 4}}{\pi^{n / 2+1 / 4}} \\
& \times(-1)^{n} \int_{\mathcal{V}} e^{-\frac{\Phi(x)+f(x)-f\left(U_{k}^{(0)}\right)}{h}}\left(\chi_{k, \varepsilon}^{\prime}\left(x_{n}\right)+\mathcal{O}_{\varepsilon}(h)\right) d x_{1} \wedge d x_{2} \wedge \ldots \wedge d x_{n}
\end{aligned}
$$

and, with $f+\Phi=f_{-}+f\left(U_{j(k)}^{(1)}\right)$, to

$$
\begin{gathered}
\left\langle\psi_{j(k)}^{(1)} \mid d_{f, h}^{(0)} \psi_{k}^{(0)}\right\rangle=h^{3 / 4-\frac{n}{2}} \frac{\sqrt{2 \pi}\left|\operatorname{det} \operatorname{Hess} f\left(U_{k}^{(0)}\right)\right|^{1 / 4}\left(\delta_{f, \partial \Omega}\left(U_{j(k)}^{(1)}\right)\right)^{1 / 4}}{\pi^{n / 2+1 / 4}} \\
\times(-1)^{n} e^{-\frac{f\left(U_{j(k)}^{(1)}\right)-f\left(U_{k}^{(0)}\right)}{h}}\left[\int_{\mathcal{V}_{-}} e^{-f_{-}(x) / h}\left(\chi_{k, \varepsilon}^{\prime}\left(x_{n}\right)+\mathcal{O}_{\varepsilon}(h)\right) d x_{1} \wedge d x_{2} \wedge \ldots \wedge d x_{n}\right] .
\end{gathered}
$$

The Laplace method, applied with $f_{-}=\left.2 f\right|_{\partial \Omega}-2 f\left(U_{j(k)}^{(1)}\right)$, gives

$$
\int_{\left|x^{\prime}\right| \leq \nu} e^{-f_{-}(x) / h} d x_{1} \wedge \ldots \wedge d x_{n-1} \sim \frac{(\pi h)^{\frac{n-1}{2}}}{\left(\delta_{f, \partial \Omega}\left(U_{j(k)}^{(1)}\right)\right)^{1 / 2}} \sum_{\ell=0}^{\infty} d_{\ell} h^{\ell}
$$

with $d_{0}=1$.

We conclude for the main term by using

$$
\int_{\mathbb{R}} \chi_{k, \varepsilon}^{\prime}\left(x_{n}\right) d x_{n}=-1
$$

\section{Corollary 6.9.}

Let $\psi_{k}^{(0)}$ and $\psi_{j}^{(1)}$ denote the $(\varepsilon, h)$-dependent and $h$-dependent quasimodes of Definition 6.2. Assume that the 1 -forms $\left(w_{j}^{(1)}\right)_{j \in\left\{1, \ldots, m_{1}\right\}}$ satisfy

$$
\left\|w_{j}^{(1)}-\psi_{j}^{(1)}\right\|=\mathcal{O}\left(e^{-\alpha / h}\right),
$$


for some $\alpha>0$ independent of $\varepsilon \in\left(0, \varepsilon_{0}\right]$. Then there exist $\varepsilon_{0}^{\prime}>0$ and $\alpha^{\prime}>0$ such that, for all $\varepsilon \in\left(0, \varepsilon_{0}^{\prime}\right]$, the estimates

$$
\left|\left\langle w_{j}^{(1)} \mid d_{f, h}^{(0)} \psi_{k}^{(0)}\right\rangle\right| \leq C_{\varepsilon} e^{-\left(f\left(U_{j(k)}^{(1)}-f\left(U_{k}^{(0)}\right)+\alpha^{\prime}\right) / h\right.}, \quad \text { if } j \neq j(k),
$$

and

$$
\left\langle w_{j(k)}^{(1)} \mid d_{f, h}^{(0)} \psi_{k}^{(0)}\right\rangle=\left\langle\psi_{j(k)}^{(1)} \mid d_{f, h}^{(0)} \psi_{k}^{(0)}\right\rangle\left(1+\mathcal{O}_{\varepsilon}\left(e^{-\alpha^{\prime} / h}\right)\right),
$$

hold for all $(k, j) \in\left\{1, \ldots, m_{0}\right\} \times\left\{1, \ldots, m_{1}\right\}$.

The proof is a straightforward consequence of Propositions 6.4 and 6.7 which give :

$$
\left\|d_{f, h}^{(0)} \psi_{k}^{(0)}\right\| \leq C_{\varepsilon} e^{-\left(f\left(U_{j(k)}^{(1)}\right)-f\left(U_{k}^{(0)}\right)-\alpha^{\prime \prime} \varepsilon\right) / h} .
$$

\section{Result and final proof.}

\subsection{Main result}

Let us first recall some notations. The local minima $U_{k}^{(0)}\left(k \in\left\{1, \ldots, m_{0}\right\}\right)$ are labelled according to Assumption 5.7, the generalized critical points with index $1, U_{j(k)}^{(1)}$ are those introduced in Definition 5.9 and the quantity $\lambda_{k}(\varepsilon, h)$ is associated with the quasimodes $\psi_{k}^{(0)}, \psi_{j(k)}^{(1)}$, in Definition 6.2 :

$$
\lambda_{k}^{a p p}(\varepsilon, h)=\left|\left\langle\psi_{j(k)}^{(1)} \mid d_{f, h}^{(0)} \psi_{k}^{(0)}\right\rangle\right|^{2} .
$$

At a generalized critical point $U$ with index 1, the Hessians $\operatorname{Hess} f(U)$ or Hess $\left.f\right|_{\partial \Omega}$ are computed in normal coordinates for the metric $g$, while considering only the tangential coordinates $x^{\prime}=\left(x_{1}, \ldots, x_{n-1}\right)$ for the second case. We refer to Remark 6.8 for the right normalization when $U \in \partial \Omega$. When $U \in \Omega, \widehat{\lambda}_{1}(U)$ denotes the negative eigenvalue of Hess $f(U)$.

\section{Theorem 7.1.}

Under Assumptions 3.1 and 5.7, the first eigenvalues $\lambda_{1}(h), \ldots, \lambda_{m_{0}}(h)$ of $\delta_{f, h}^{D T,(0)}$ admit the following asymptotic expansion. There exist $\varepsilon_{0}>0$ and $\alpha>0$, such that, for any $\varepsilon \in\left(0, \varepsilon_{0}\right]$,

$$
\forall k \in\left\{1, \ldots, m_{0}\right\}, \quad \lambda_{k}(h)=\lambda_{k}^{a p p}(\varepsilon, h)\left(1+\mathcal{O}_{\varepsilon}\left(e^{-\alpha / h}\right)\right) .
$$


Moreover there exist sequences $\left(c_{k, m}\right)_{m \in \mathbb{N}^{*}}$ such that, for any $\varepsilon \in\left(0, \varepsilon_{0}\right]$,

$$
\begin{aligned}
\lambda_{k}^{a p p}(\varepsilon, h)=\frac{h}{\pi}\left|\widehat{\lambda}_{1}\left(U_{j(k)}^{(1)}\right)\right| & \sqrt{\frac{\left|\operatorname{det}\left(\operatorname{Hess} f\left(U_{k}^{(0)}\right)\right)\right|}{\left|\operatorname{det}\left(\operatorname{Hess} f\left(U_{j(k)}^{(1)}\right)\right)\right|}}\left(1+h c_{k}^{1}(h)\right) \\
& \times \exp -\frac{2}{h}\left(f\left(U_{j(k)}^{(1)}\right)-f\left(U_{k}^{(0)}\right)\right), \quad \text { if } U_{j(k)}^{(1)} \in \Omega,
\end{aligned}
$$

and

$$
\begin{array}{r}
\lambda_{k}^{a p p}(\varepsilon, h)=\frac{2 h^{1 / 2}\left|\nabla f\left(U_{j(k)}^{(1)}\right)\right|}{\pi^{1 / 2}} \sqrt{\frac{\left|\operatorname{det}\left(\operatorname{Hess} f\left(U_{k}^{(0)}\right)\right)\right|}{\left|\operatorname{det}\left(\left.\operatorname{Hess} f\right|_{\partial \Omega}\left(U_{j(k)}^{(1)}\right)\right)\right|}}\left(1+h c_{k}^{1}(h)\right) \\
\times \exp -\frac{2}{h}\left(f\left(U_{j(k)}^{(1)}\right)-f\left(U_{k}^{(0)}\right)\right), \quad \text { if } U_{j(k)}^{(1)} \in \partial \Omega,
\end{array}
$$

with $c_{k}^{1}(h) \sim \sum_{m=0}^{\infty} h^{m} c_{k, m}$.

This theorem implies the theorem announced in the first section. The core of the proof is essentially the same as in the case without boundary treated in $[\mathrm{HKN}]$. We give it for the sake of completeness. The main idea is that the eigenvalues of $\left.\Delta_{f, h}^{D T,(0)}\right|_{F^{(0)}}=\beta_{f, h}^{(0) *} \beta_{f, h}^{(0)}$ are the singular values of $\beta_{f, h}^{(0)}$. The Fan inequality for singular values permits to control the relative error for all singular values, when the matrix of $\beta_{f, h}^{(0)}$ is expressed in different bases. The proof will be done in two steps.

\subsection{Finite dimensional reduction}

Theorem 3.3 and the results of Section 5 lead to the

\section{Proposition 7.2.}

There exist $\alpha, \alpha^{\prime}>0$ such that :

$$
1_{\left[0, h^{3 / 2}\right)}\left(\Delta_{f, h}^{D T,(\ell)}\right)=1_{\left[0, e^{-\alpha / h}\right)}\left(\Delta_{f, h}^{D T,(\ell)}\right), \text { for } \ell=0,1 .
$$

Moreover if one sets

$$
\forall i \in\left\{1, \ldots, m_{\ell}\right\}, \quad v_{i}^{(\ell)}=1_{\left[0, h^{3 / 2}\right)}\left(\Delta_{f, h}^{D T,(\ell)}\right) \psi_{i}^{(\ell)},
$$


where the $\psi_{i}^{(\ell)}$ are the $(\varepsilon, h)$ - and $h$-dependent quasimodes introduced in Definition 6.2, the system $\left(v_{i}^{(\ell)}\right)_{i \in\left\{1, \ldots, m_{\ell}\right\}}$ is a basis of $F^{(\ell)}$ such that :

$$
\begin{aligned}
& \text { 1) } \quad \forall i \in\left\{1, \ldots, m_{\ell}\right\}, \quad\left\|v_{i}^{(\ell)}-\psi_{i}^{(\ell)}\right\|=\mathcal{O}\left(e^{-\alpha^{\prime} / h}\right) ; \\
& \text { 2) } \quad V^{(\ell)}:=\left(\left\langle v_{i}^{(\ell)} \mid v_{i^{\prime}}^{(\ell)}\right\rangle\right)_{i, i^{\prime} \in\left\{1, \ldots, m_{\ell}\right\}}=\operatorname{Id}_{\mathbb{C}^{m_{\ell}}}+\mathcal{O}\left(e^{-\alpha^{\prime} / h}\right) .
\end{aligned}
$$

\section{Remark 7.3.}

Note that here again we omit the $(\varepsilon, h)$-dependence (resp. h-dependence) of the functions $v_{k}^{(0)}$ (resp. 1-forms $v_{j}^{(1)}$ ) in the notation.

Proof.

Let $\ell \in\{0,1\}$ and $i \in\left\{1, \ldots, m_{\ell}\right\}$. According to Lemma 2.8, Corollary 6.5 and Proposition 6.6, $\left\|1_{\left[h^{3 / 2} / 2,+\infty\right)}\left(\Delta_{f, h}^{D T,(\ell)}\right) \psi_{i}^{(\ell)}\right\|$ is estimated from above by $\mathcal{O}\left(e^{-\alpha^{\prime} / h}\right)$. The second estimate then comes from the almost orthonormality of $\left(\psi_{i}^{(\ell)}\right)_{i \in\left\{1, \ldots, m_{\ell}\right\}}$. Since we know by Proposition 3.6-iii) that $F^{(\ell)}$ has dimension $m_{\ell}$, the system $\left(v_{i}^{(\ell)}\right)_{i \in\left\{1, \ldots, m_{\ell}\right\}}$ is a basis of $F^{(\ell)}$. We conclude with

$$
\left\langle\Delta_{f, h}^{D T,(\ell)} v_{i}^{(\ell)} \mid v_{i}^{(\ell)}\right\rangle \leq\left\langle\Delta_{f, h}^{D T,(\ell)} \psi_{i}^{(\ell)} \mid \psi_{i}^{(\ell)}\right\rangle \leq e^{-2 \alpha / h} .
$$

\section{Definition 7.4.}

The basis $\left(e_{i}^{(\ell)}\right)_{i \in\left\{1, \ldots, m_{\ell}\right\}}$ of $F^{(\ell)}$ is the orthonormal basis derived from $\left(v_{i}^{(\ell)}\right)_{i \in\left\{1, \ldots, m_{\ell}\right\}}$ by the Gram-Schmidt orthonormalization procedure

$$
e_{i}^{(\ell)}=\sum_{i^{\prime}}\left[\left(V^{(\ell)}\right)^{-1 / 2}\right]_{i i^{\prime}} v_{i^{\prime}}^{(\ell)}
$$

The $m_{1} \times m_{0}$ matrix $\mathcal{M}$ is the matrix of $\beta_{f, h}^{(0)}$ in the bases $\left(e_{k}^{(0)}\right)_{k \in\left\{1, \ldots, m_{0}\right\}}$ and $\left(e_{j}^{(1)}\right)_{j \in\left\{1, \ldots, m_{1}\right\}}$. Its square $\mathcal{M}^{*} \mathcal{M}$ is called the interaction matrix.

According to (2.21), the $m_{0}$ eigenvalues of the restricted Witten Laplacian $\left.\Delta_{f, h}^{D T,(0)}\right|_{F^{(0)}}=\beta_{f, h}^{(0) *} \beta_{f, h}^{(0)}$ are the eigenvalues of the interaction matrix $\mathcal{M}^{*} \mathcal{M}$.

\footnotetext{
${ }^{5}$ We recall from $(1.9)$ that $\beta_{f, h}^{(0)}$ is defined from $F^{(0)}$ into $F^{(1)}$ by the restriction of $d_{f, h}^{(0)}$ to $F^{(0)}$.
} 
Hence it is theoretically possible to determine the low lying eigenvalues of $\Delta_{f, h}^{D T,(0)}$ by analyzing the matrix $\mathcal{M}$. The problem is that the coefficients of the matrix $\mathcal{M}$ are not known at this level accurately enough in order to split the different exponentially small scales. Like in $[\mathrm{HKN}]$, we will work with the matrix

$$
\mathcal{I}=\left(\left\langle v_{j}^{(1)} \mid \beta_{f, h}^{(0)} v_{k}^{(0)}\right\rangle\right)_{(j, k) \in\left\{1, \ldots, m_{1}\right\} \times\left\{1, \ldots, m_{0}\right\}} .
$$

of the map $\beta_{f, h}^{(0)}$, written in the bases $\left(v_{k}^{(0)}\right)_{k \in\left\{1, \ldots, m_{0}\right\}}$ in $F^{(0)}$ and $\left(v_{j}^{(1), *}\right)_{j \in\left\{1, \ldots, m_{1}\right\}}$ dual to $\left(v_{j}^{(1)}\right)_{j \in\left\{1, \ldots, m_{1}\right\}}$ in $F^{(1)}$. This permits to use directly all the accurate information that we have on the quasimodes $\psi_{i}^{(\ell)}$. The fact that these bases are not orthonormal does not make any problem if one notices that the eigenvalues of $\mathcal{M}^{*} \mathcal{M}$ are the squares of the singular values of $\beta_{f, h}^{(0)}$.

\subsection{Singular values and induction.}

The first eigenvalues $\lambda_{k}(h), 1 \leq k \leq m_{0}$, of $\Delta_{f, h}^{D T,(0)}$ are the squares of the singular values ${ }^{6} \mu_{m_{0}+1-k}(\mathcal{M})$ of $\mathcal{M}$. In other words,

$$
\lambda_{k}(h)=\left[\mu_{m_{0}+1-k}\left(\beta_{f, h}^{(0)}\right)\right]^{2} .
$$

We will use the simple consequence of the Fan inequalities (see [Sim1], $[\mathrm{GoKr}])$ :

\section{Proposition 7.5.}

For any matrices $A$ and $B$ such that,

$$
\max \left\{\|B\|,\left\|B^{-1}\right\|\right\} \leq 1+\rho,
$$

the singular values of $A$ and $A B$ satisfy

$$
\frac{\mu_{k}(A)}{(1+\rho)} \leq \mu_{k}(A B) \leq(1+\rho) \mu_{k}(A)
$$

and the same holds with $A B$ replaced by $B A$.

\footnotetext{
${ }^{6}$ The singular values $\mu_{k}(A)$ are numbered here as usual in the decreasing order with $\mu_{1}(A)=\|A\|$.
} 
Hence a small change of bases induces a small relative variation of the singular values and it is not necessary to work with orthonormal bases in order to estimate the singular values.

For example, we have, for any $k \in\left\{1, \ldots, m_{0}\right\}$,

$$
\mu_{k}\left(\beta_{f, h}^{(0)}\right)=\mu_{k}(\mathcal{M})=\mu_{k}(\mathcal{I})\left(1+\mathcal{O}\left(e^{-\alpha / h}\right)\right),
$$

where $\mathcal{I}$ is the matrix of the map $\beta_{f, h}^{(0)}$ introduced in (7.2).

We will construct by reverse induction on $K$, from $m_{0}$ down to $K=0$, two bases $\left(v_{k, K}^{(0)}\right)_{k \in\left\{1, \ldots, m_{0}\right\}}$ of $F^{(0)}$ and of $F^{(1)}\left(v_{j, K}^{(1)}\right)_{j \in\left\{1, \ldots, m_{1}\right\}}$ so that the following properties hold for $\varepsilon \in\left(0, \varepsilon_{0}\right]$ and some $\alpha>0$ independent of $\varepsilon$.

1) The systems $\left(v_{k, K}^{(0)}\right)_{K<k \leq m_{0}}$ and $\left(v_{j(k), K}^{(1)}\right)_{K<k \leq m_{0}}$ are orthonormal.

We then set

$F_{K}^{(0)}=\operatorname{Span}\left\{v_{k, K}^{(0)}, K<k \leq m_{0}\right\} \quad$ and $\quad F_{K}^{(1)}=\operatorname{Span}\left\{v_{j(k), K}^{(1)}, K<k \leq m_{0}\right\}$.

2) For $1 \leq k \leq K, v_{k, K}^{(0)}$ belongs to $\left(F_{K}^{(0)}\right)^{\perp}$ and for $j \notin\left\{j(k), K<k \leq m_{0}\right\}$, $v_{j, K}^{(1)}$ belongs to $\left(F_{K}^{(1)}\right)^{\perp}$.

3) The estimates,

$$
\forall i \in\left\{1, \ldots, m_{\ell}\right\}, \quad\left\|v_{i, K}^{(\ell)}-\psi_{i}^{(\ell)}\right\|=\mathcal{O}_{\varepsilon}\left(e^{-\alpha / h}\right),
$$

hold for $\ell=0,1$.

4) For $K<k \leq m_{0}$, the equalities

$$
\beta_{f, h}^{(0)} v_{k, K}^{(0)}=\nu_{k} v_{j(k), K}^{(1)} \quad \text { and } \quad \Delta_{f, h}^{D T,(0)} v_{k, K}^{(0)}=\nu_{k}^{2} v_{k, K}^{(0)}
$$

hold with

$$
\nu_{k}=\left\langle\psi_{j(k)}^{(1)} \mid d_{f, h}^{(0)} \psi_{k}^{(0)}\right\rangle\left(1+\mathcal{O}_{\varepsilon}\left(e^{-\alpha / h}\right)\right) .
$$

They imply, observing also that $\nu_{k} \neq 0$,

$$
\Delta_{f, h}^{D T,(\ell)} F_{K}^{(\ell)} \subset F_{K}^{(\ell)}, \quad \ell \in\{0,1\} .
$$

5) For all $j \notin\left\{j(k), K<k \leq m_{0}\right\}$ and all $k \in\{1, \ldots, K\}$, we have

$$
\left\langle v_{j, K}^{(1)} \mid \beta_{f, h}^{(0)} v_{k, K}^{(0)}\right\rangle=\left\langle v_{j, K}^{(1)} \mid d_{f, h}^{(0)} \psi_{k}^{(0)}\right\rangle .
$$


We recall that the $\psi_{i}^{(\ell)}$ and the $v_{i}^{(\ell)}$ depend on $h \in\left(0, h_{0}\right]$ and $\varepsilon \in\left(0, \varepsilon_{0}\right]$, while $\alpha>0$ enters in the exponential estimates. The parameters $\varepsilon_{0}>0$ and $\alpha>0$ belong to intervals which have to be reduced each time that one refers to Corollary 6.9. This is done a finite number of times at each step of the induction.

Initialization : the case $K=m_{0}$.

We take $v_{k, m_{0}}^{(0)}=v_{k}^{(0)}$ and $v_{j, m_{0}}^{(1)}=v_{j}^{(1)}$ according to the definition of the previous section. Conditions 1), 2) and 4) are empty. Conditions 2) and 3) are given in Proposition 7.2. For Condition 5), we write

$$
\begin{array}{r}
\left\langle v_{j}^{(1)} \mid \beta_{f, h}^{(0)} v_{k}^{(0)}\right\rangle=\left\langle 1_{\left[0, h^{3 / 2}\right)}\left(\Delta_{f, h}^{D T,(1)}\right) v_{j}^{(1)} \mid d_{f, h}^{(0)} 1_{\left[0, h^{3 / 2}\right)}\left(\Delta_{f, h}^{D T,(0)}\right) \psi_{k}^{(0)}\right\rangle \\
=\left\langle 1_{\left[0, h^{3 / 2}\right)}\left(\Delta_{f, h}^{D T,(1)}\right) v_{j}^{(1)} \mid d_{f, h}^{(0)} \psi_{k}^{(0)}\right\rangle=\left\langle v_{j}^{(1)} \mid d_{f, h}^{(0)} \psi_{k}^{(0)}\right\rangle
\end{array}
$$

\section{Recursion : from $K$ to $K-1$.}

Assume that the result is true for $K>0$. Conditions 1) and 4) say that the quantities $\left|\nu_{k}\right|, K<k \leq m_{0}$ are singular values of $\beta_{f, h}^{(0)}\left(\nu_{k}^{2}\right.$ is an eigenvalue of $\left.\left.\Delta_{f, h}^{D T,(0)}\right|_{F^{(0)}}\right)$. Moreover the estimate,

$$
\nu_{k}=\left\langle\psi_{j(k)}^{(1)} \mid d_{f, h}^{(0)} \psi_{k}^{(0)}\right\rangle\left(1+\mathcal{O}_{\varepsilon}\left(e^{-\alpha / h}\right)\right),
$$

and Proposition 6.7 imply

$$
\left|\nu_{k}\right| \geq C_{\varepsilon} h^{1 / 2} e^{-\left(f\left(U_{j(K+1)}\right)-f\left(U_{K+1}^{(0)}\right)\right) / h} \geq C_{\varepsilon} e^{-\left(f\left(U_{j(K)}\right)-f\left(U_{K}^{(0)}\right)-2 \alpha_{1}\right) / h},
$$

with $\alpha_{1}$ independent of $\varepsilon>0$.

Let us consider the dual basis $\left(v_{j, K}^{(1), *}\right)$ in $F^{(1)}$. For $j=j(k), K<k \leq m_{0}$, $v_{j, K}^{(1), *}$ equals $v_{j, K}^{(1)}$ and consequently

$$
\left\|v_{j, K}^{(1), *}-\psi_{j}^{(1)}\right\|=\mathcal{O}_{\varepsilon}\left(e^{-\alpha / h}\right) .
$$

The matrix of $\beta_{f, h}^{(0)}:\left(F_{K}^{(0)}\right)^{\perp} \rightarrow\left(F_{K}^{(1)}\right)^{\perp}$ in the bases $\left(v_{k, K}^{(0)}\right)_{1 \leq k \leq K}$ and $\left(v_{j, K}^{(1), *}\right)_{j \notin\left\{j(k), K<k \leq m_{0}\right\}}$ equals

$$
\left(\left\langle v_{j, K}^{(1)} \mid \beta_{f, h}^{(0)} v_{k, K}^{(0)}\right\rangle\right)_{j \notin\left\{j(k), K<k \leq m_{0}\right\}, 1 \leq k \leq K} .
$$


Conditions 3) and 5) and Corollary 6.9 lead to

$$
\left\|\left.\beta_{f, h}\right|_{\left(F_{K}^{(0)}\right)^{\perp}}\right\|=\mathcal{O}_{\varepsilon}\left(e^{-\left(f\left(U_{j(K)}\right)-f\left(U_{K}^{(0)}\right)-\alpha_{1}\right) / h}\right) .
$$

Hence the quantity $\left|\nu_{k}\right|, K<k \leq m_{0}$ are the first largest singular values of $\beta_{f, h}^{(0)}$,

$$
\forall k \in\left\{K+1, \ldots, m_{0}\right\}, \quad\left|\nu_{k}\right|=\mu_{m_{0}+1-k}\left(\beta_{f, h}^{(0)}\right)=\sqrt{\lambda_{k}(h)},
$$

and we have

$$
\sqrt{\lambda_{K}(h)}=\mu_{m_{0}+1-K}\left(\beta_{f, h}^{(0)}\right)=\left\|\left.\beta_{f, h}^{(0)}\right|_{\left(F_{K}^{(0)}\right)^{\perp}}\right\|
$$

Let us now consider more carefully $\left.\beta_{f, h}^{(0)}\right|_{\left(F_{K}^{(0)}\right)^{\perp}}$ and its matrix (7.5) in the bases $\left(v_{k, K}^{(0)}\right)_{1 \leq k \leq K},\left(v_{j, K}^{(1), *}\right)_{j \notin\left\{j(k), K<k \leq m_{0}\right\}}$. With the same arguments as above relying on Corollary 6.9 and Conditions 3) and 5), its coefficients have the form

$$
\left\langle\psi_{j(K)}^{(1)} \mid d_{f, h}^{(0)} \psi_{K}^{(0)}\right\rangle\left(\delta_{j(K), j} \delta_{K, k}+\mathcal{O}_{\varepsilon}\left(e^{-\alpha_{2} / h}\right)\right) .
$$

Since the two bases are $\mathcal{O}_{\varepsilon}\left(e^{-\alpha / h}\right)$-close to orthonormal bases, we obtain

$$
\sqrt{\lambda_{K}(h)}=\left|\left\langle\psi_{j(K)}^{(1)} \mid d_{f, h}^{(0)} \psi_{K}^{(0)}\right\rangle\right|\left(1+\mathcal{O}_{\varepsilon}\left(e^{-\alpha_{3} / h}\right)\right) .
$$

We set

$$
\nu_{K}=\frac{\left\langle\psi_{j(K)}^{(1)} \mid d_{f, h}^{(0)} \psi_{K}^{(0)}\right\rangle}{\left|\left\langle\psi_{j(K)}^{(1)} \mid d_{f, h}^{(0)} \psi_{K}^{(0)}\right\rangle\right|} \sqrt{\lambda_{K}(h)}
$$

We have

$$
\beta_{f, h}^{(0)} v_{K, K}^{(0)}=\nu_{K} v_{j(K), K}^{(1), *}+\mathcal{O}_{\varepsilon}\left(\nu_{K} e^{-\alpha_{4} / h}\right) .
$$

We next define the new bases $\left(v_{k, K-1}^{(0)}\right)$ and $\left(v_{j, K-1}^{(1)}\right)$.

Of course we keep $v_{k, K-1}^{(0)}=v_{k, K}^{(0)}$ and $v_{j(k), K-1}^{(1)}=v_{j(k), K}^{(1)}$ for $K<k \leq m_{0}$. We then take

$$
v_{K, K-1}^{(0)}=\left\|1_{\left\{\lambda_{K}\right\}}\left(\Delta_{f, h}^{D T,(0)}\right) v_{K, K}\right\|^{-1} 1_{\left\{\lambda_{K}\right\}}\left(\Delta_{f, h}^{D T,(0)}\right) v_{K, K},
$$

and

$$
v_{j(K), K-1}^{(1)}=\frac{1}{\nu_{K}} \beta_{f, h}^{(0)} v_{K, K-1}^{(0)} .
$$


For $1 \leq k \leq K-1$ and $j \notin\left\{j(k), K-1<k \leq m_{0}\right\}$, we take

and

$$
v_{k, K-1}^{(0)}=v_{k, K}^{(0)}-\left\langle v_{k, K}^{(0)} \mid v_{K, K-1}^{(0)}\right\rangle v_{K, K-1}^{(0)}
$$

$$
v_{j, K-1}^{(1)}=v_{j, K}^{(0)}-\left\langle v_{j, K}^{(1)} \mid v_{j(K), K-1}^{(1)}\right\rangle v_{j(K), K-1}^{(1)} .
$$

By construction, conditions 1), 2) and 4) are satisfied by these new bases. Condition 3) will be satisfied as well if $\left\|v_{K, K}^{(0)}-v_{K, K-1}^{(0)}\right\|=\mathcal{O}_{\varepsilon}\left(e^{-\alpha_{5} / h}\right)$ holds.

The identity (7.6) gives

$$
\forall k \in\{1, \ldots, K\}, \quad v_{k, K}^{(0)}=1_{\left[0, \lambda_{K}\right]}\left(\Delta_{f, h}^{D T,(0)}\right) v_{k, K}^{(0)}
$$

Moreover Corollary 6.9 yields

$\forall k \in\{1, \ldots, K-1\}, \forall j \in\left\{1, \ldots, m_{1}\right\}, \quad\left|\left\langle v_{j, K}^{(1)} \mid \beta_{f, h}^{(0)} v_{k, K}^{(0)}\right\rangle\right|=\mathcal{O}_{\varepsilon}\left(\sqrt{\lambda_{K}} e^{-\alpha_{6} / h}\right)$.

Like in the proof of Proposition 7.2, we obtain for some $\alpha_{7}>0$

$$
1_{\left[0, \lambda_{K}\right)}\left(\Delta_{f, h}^{D T,(0)}\right)=1_{\left[0, \lambda_{K} e^{-\alpha_{7} / h}\right)}\left(\Delta_{f, h}^{D T,(0)}\right) .
$$

We now write, by spectral decomposition and using (7.11) and (7.10),

$$
\begin{aligned}
\lambda_{K}\left\|1_{\left\{\lambda_{K}\right\}}\left(\Delta_{f, h}^{D T,(0)}\right) v_{K, K}^{(0)}\right\|^{2}+\mathcal{O}_{\varepsilon}\left(\lambda_{K} e^{-\alpha_{7} / h}\right) & \left\|1_{\left[0, \lambda_{K}\right)}\left(\Delta_{f, h}^{D T,(0)}\right) v_{K, K}^{(0)}\right\|^{2} \\
& =\left\langle\Delta_{f, h}^{D T,(0)} v_{K, K}^{(0)} \mid v_{K, K}^{(0)}\right\rangle
\end{aligned}
$$

and observe that by (7.9)

$$
\left\langle\Delta_{f, h}^{D T,(0)} v_{K, K}^{(0)} \mid v_{K, K}^{(0)}\right\rangle=\left\|\beta_{f, h}^{(0)} v_{K, K}^{(0)}\right\|^{2}=\lambda_{K}\left(1+\mathcal{O}_{\varepsilon}\left(e^{-\alpha_{4} / 2 h}\right)\right)
$$

Hence we obtain

$$
\left\|1_{\left\{\lambda_{K}\right\}}\left(\Delta_{f, h}^{D T,(0)}\right) v_{K, K}^{(0)}\right\|=1+\mathcal{O}_{\varepsilon}\left(e^{-\alpha_{8} / h}\right) .
$$

We conclude with

$$
\begin{aligned}
\left\|1_{\left[0, \lambda_{K}\right)}\left(\Delta_{f, h}^{D T,(0)}\right) v_{K, K}^{(0)}\right\|^{2} & =\left\|v_{K, K}^{(0)}\right\|^{2}-\left\|1_{\left\{\lambda_{K}\right\}}\left(\Delta_{f, h}^{D T,(0)}\right) v_{K, K}^{(0)}\right\|^{2} \\
& =\mathcal{O}_{\varepsilon}\left(e^{-2 \alpha / h}\right)+\mathcal{O}_{\varepsilon}\left(e^{-2 \alpha_{8} / h}\right) .
\end{aligned}
$$


We have proved

$$
\left\|v_{K, K}^{(0)}-v_{K, K-1}^{(0)}\right\|=\mathcal{O}_{\varepsilon}\left(e^{-\alpha_{5} / h}\right) .
$$

This implies

$$
\begin{aligned}
& \left\|\beta_{f, h}^{(0)} v_{K, K}^{(0)}-\nu_{K} v_{j(K), K-1}^{(1)}\right\|=\left\|\beta_{f, h}^{(0)} v_{K, K}^{(0)}-\beta_{f, h}^{(0)} v_{K, K-1}^{(0)}\right\| \\
& =\left\|\beta_{f, h}^{(0)} 1_{\left[0, \lambda_{K}\right]}\left(\Delta_{f, h}^{D T,(0)}\right)\left(v_{K, K}^{(0)}-v_{K, K-1}^{(0)}\right)\right\| \\
& =\mathcal{O}_{\varepsilon}\left(\sqrt{\lambda_{K}} e^{-\alpha_{5} / h}\right) \text {, }
\end{aligned}
$$

while we have

$$
\left\|\beta_{f, h}^{(0)} v_{K, K}^{(0)}-\nu_{K} v_{j(K), K}^{(1), *}\right\|=\mathcal{O}_{\varepsilon}\left(\nu_{K} e^{-\alpha_{4} / h}\right)
$$

The almost orthonormality of $\left(v_{j, K}^{(1)}\right)_{j \in\left\{1, \ldots, m_{0}\right\}}$ inherited from Condition 3 ) and the almost orthogonality of $\left(\psi_{j}^{(1)}\right)_{\left\{1, \ldots, m_{1}\right\}}$ imply

$$
\left\|v_{j(K), K}^{(1)}-v_{j(K), K}^{(1), *}\right\|=\mathcal{O}_{\varepsilon}\left(e^{-\alpha / 2 h}\right) .
$$

This yields

$$
\left\|v_{j(K), K-1}^{(1)}-v_{j(K), K}^{(1)}\right\|=\mathcal{O}_{\varepsilon}\left(e^{-\alpha_{9} / h}\right) .
$$

\section{Let us verify Condition 5) for the new bases.}

For $k \in\{1, \ldots, K-1\}$, the construction of the new bases and the induction gives

$$
\begin{aligned}
v_{k, K-1}^{(0)} & =v_{k, K}^{(0)}-\left\langle v_{k, K}^{(0)} \mid v_{K, K-1}^{(0)}\right\rangle v_{K, K-1}^{(0)} \\
= & v_{k, m_{0}}^{(0)}-\sum_{K \leq K^{\prime} \leq m_{0}} t_{k, K^{\prime}} v_{K^{\prime}, K^{\prime}-1}^{(0)} \\
= & v_{k}^{(0)}-\sum_{K \leq K^{\prime} \leq m_{0}} t_{k, K^{\prime}} v_{K^{\prime}, K-1}^{(0)}
\end{aligned}
$$

with $t_{k, K^{\prime}}:=\left\langle v_{k, K^{\prime}}^{(0)} \mid v_{K^{\prime}, K^{\prime}-1}^{(0)}\right\rangle$.

Hence we get, with $v_{k}^{(0)}=1_{\left[0, h^{3 / 2}\right)}\left(\Delta_{f, h}^{D T,(0)}\right) \psi_{k}^{(0)}$,

$$
\begin{aligned}
\beta_{f, h}^{(0)} v_{k, K-1}^{(0)} & =\beta_{f, h}^{(0)} v_{k}^{(0)}-\sum_{K \leq K^{\prime} \leq m_{0}} t_{k, K^{\prime}} \beta_{f, h}^{(0)} v_{K^{\prime}, K-1}^{(0)} \\
& =1_{\left[0, h^{3 / 2}\right)}\left(\Delta_{f, h}^{D T,(1)}\right) d_{f, h}^{(0)} \psi_{k}^{(0)}-\sum_{K \leq K^{\prime} \leq m_{0}} t_{k, K^{\prime}} \nu_{K^{\prime}} v_{j\left(K^{\prime}\right), K-1}^{(1)} .
\end{aligned}
$$


Meanwhile, for $j \notin\left\{j(k), K-1<k \leq m_{0}\right\}$, the vectors $v_{j, K-1}^{(1)}$ were constructed such that

$$
v_{j, K-1}^{(1)} \in\left(F_{K-1}^{(1)}\right)^{\perp}=\left(\operatorname{Span}\left\{v_{j(K), K-1}^{(1)}, \ldots, v_{j\left(m_{0}\right), K-1}^{(1)}\right\}\right)^{\perp} .
$$

We obtain, for all $k \in\{1, \ldots, K-1\}$ and all $j \notin\left\{j(k), K-1<k \leq m_{0}\right\}$,

$$
\begin{aligned}
\left\langle v_{j, K-1}^{(1)} \mid \beta_{f, h}^{(0)} v_{k, K-1}^{(0)}\right\rangle & =\left\langle 1_{\left[0, h^{3 / 2}\right)}\left(\Delta_{f, h}^{D T,(1)}\right) v_{j, K-1}^{(1)} \mid d_{f, h}^{(0)} \psi_{k}^{(0)}\right\rangle \\
& =\left\langle v_{j, K-1}^{(1)} \mid d_{f, h}^{(0)} \psi_{k}^{(0)}\right\rangle .
\end{aligned}
$$

Conclusion for $K=0$ : When $K=0$, we obtain an orthonormal basis $\left(v_{k, 0}^{(0)}\right)_{0<k \leq m_{0}}$ of $F_{0}^{(0)}=F^{(0)}$ and an orthonormal basis $\left(v_{j(k)}^{(1)}\right)_{0<k \leq m_{0}}$ of $F_{0}^{(1)} \subset$ $F^{(1)}$ such that for $\epsilon \in\left(0, \epsilon_{0}\right)$ and $\alpha>0$ independent of $\epsilon$,

$$
\begin{array}{ll}
\forall k \in\left\{1, \ldots, m_{0}\right\}, \quad & \beta_{f, h}^{(0)} v_{k, 0}^{(0)}=\nu_{k} v_{j(k), 0}^{(1)}, \\
& \left|\nu_{k}\right|=\mu_{m_{0}+1-k}\left(\beta_{f, h}^{(0)}\right) . \\
& \nu_{k}=\left\langle\psi_{j(k)}^{(1)} \mid d_{f, h}^{(0)} \psi_{k}^{(0)}\right\rangle\left(1+\mathcal{O}_{\epsilon}\left(e^{-\alpha / h}\right)\right) .
\end{array}
$$

\section{Acknowledgments.}

We would like to thank J.M. Bismut, M. Dauge, M. Klein, F. Laudenbach and J. Sjöstrand for enlightening discussions. The first author acknowledges the support of the European Union through the IHP network of the EU N0 HPRN-CT-2002-00277 and of the European Science Foundation (programme SPECT). The second author benefitted from the support of the ACI-Jeunes chercheurs "Systèmes hors-équilibre quantiques et classiques".

\section{References}

[Bis] J.M. Bismut. The Witten complex and the degenerate Morse inequalities. J. Differ. Geom. 23, p. 207-240 (1986).

[BEGK] A. Bovier, M. Eckhoff, V. Gayrard, and M. Klein : Metastability in reversible diffusion processes I. Sharp asymptotics for capacities and exit times. To appear in JEMS (2004). 
[BoGayKl] A. Bovier, V. Gayrard, and M. Klein. Metastability in reversible diffusion processes II Precise asymptotics for small eigenvalues. Preprint 2002 (new version in 2004). To appear in JEMS (2004).

[BoHe] C. Bolley and B. Helffer. Application of semi-classical analysis to the asymptotic study of the supercooling field of a superconducting material. Ann. Inst. H. Poincaré Phys. Théor. 58 (2), p. 189-233 (1991).

[BruLe] J. Brüning and M. Lesch. Hilbert complexes. J. Funct. Anal. 108 (1), p. 88-132 (1992).

[Bur] D. Burghelea. Lectures on Witten-Helffer-Sjöstrand theory. Gen. Math. 5, p. 85-99 (1997).

[CL] Kung Ching Chang and Jiaquan Liu. A cohomology complex for manifolds with boundary. Topological methods in non linear analysis. Volume 5, p. 325-340 (1995).

[CFKS] H.L Cycon, R.G Froese, W. Kirsch, and B. Simon. Schrödinger operators with application to quantum mechanics and global geometry. Text and Monographs in Physics. Springer-Verlag (1987).

[Da] M. Dauge. Elliptic boundary value problems on corner domains. Smoothness and asymptotic solutions. Lect. Notes in Math. 1341, Springer-Verlag (1988).

[DiSj] M. Dimassi and J. Sjöstrand. Spectral Asymptotics in the semiclassical limit. London Mathematical Society. Lecture Note Series 268. Cambridge University Press (1999).

[Du] G.F.D. Duff. Differential forms in manifolds with boundary. Ann. of Math. 56, p. 115-127 (1952).

[DS] G.F.D. Duff and D.C. Spencer. Harmonic tensors on Riemannian manifolds with boundary. Ann. of Math. 56, p. 128-156 (1952).

[FrWe] M.I. Freidlin and A.D. Wentzell. Random perturbations of $d y$ namical systems. Transl. from the Russian by Joseph Szuecs. 2nd ed. Grundlehren der Mathematischen Wissenschaften. 260. New York (1998). 
[Gol] S.I. Goldberg. Curvature and Homology.

Dover books in Mathematics, 3rd edition (1998).

[GoKr] I.C. Gohberg and M.G. Krejn. Introduction à la théorie des opérateurs linéaires non auto-adjoints dans un espace hilbertien.

Monographies Universitaires de Mathématiques, No. 39. Dunod, Paris (1971).

[GM] P.A. Griffiths and J.W. Morgan. Rational homotopy theory and differential forms. Birkhäuser (1981).

[Gri] P. Grisvard. Elliptic problems in nonsmooth domains. Monographs and Studies in Mathematics 24. Pitman (Advanced Publishing Program) (1985).

[Gu] P. Guérini. Prescription du spectre du Laplacien de Hodge-de Rham. Annales de l'ENS, Vol. 37 (2), p. 270-303 (2004).

[He1] B. Helffer. Etude du Laplacien de Witten associé à une fonction de Morse dégénérée, Publications de l'université de Nantes, Séminaire EDP 1987-88.

[He2] B. Helffer. Introduction to the semi-classical Analysis for the Schrödinger operator and applications. Springer Verlag. Lecture Notes in Math. $\mathrm{n}^{\circ} 1336$ (1988).

[He3] B. Helffer. Semi-classical analysis, Witten Laplacians and statistical mechanics. World Scientific (2002).

[HKN] B. Helffer, M. Klein, and F. Nier. Quantitative analysis of metastability in reversible diffusion processes via a Witten complex approach. Preprint 2004.

[HelNi] B. Helffer and F. Nier. Hypoellipticity and spectral theory for Fokker-Planck operators and Witten Laplacians. Prépublication 03-25 de l'IRMAR, Univ. Rennes 1 (sept. 2003).

[HelSj1] B. Helffer and J. Sjöstrand. Multiple wells in the semi-classical limit I, Comm. Partial Differential Equations 9 (4), p. 337-408, (1984). 
[HelSj2] B. Helffer and J. Sjöstrand. Puits multiples en limite semiclassique II -Interaction moléculaire-Symétries-Perturbations. Ann. Inst. H. Poincaré Phys. Théor. 42 (2), p. 127-212 (1985).

[HelSj3] B. Helffer and J. Sjöstrand. Multiple wells in the semi-classical limit III. Math. Nachr. 124, p. 263-313 (1985).

[HelSj4] B. Helffer and J. Sjöstrand. Puits multiples en limite semi-classique IV -Etude du complexe de Witten -. Comm. Partial Differential Equations 10 (3), p. 245-340 (1985).

[HelSj5] B. Helffer and J. Sjöstrand. Puits multiples en limite semi-classique V - Etude des minipuits-. Current topics in partial differential equations, p. 133-186, Kinokuniya, Tokyo (1986).

[HolKusStr] R. Holley, S. Kusuoka, and D. Stroock. Asymptotics of the spectral gap with applications to the theory of simulated annealing. J. Funct. Anal. 83 (2), p. 333-347 (1989).

[Hö] L. Hörmander. The Analysis of linear Partial Differential Operators, Grundlehren der Mathematischen Wissenschaften, Springer Verlag (1984).

[Jo] J. Johnsen. On the spectral properties of Witten Laplacians, their range projections and Brascamp-Lieb's inequality. Integral Equations Operator Theory 36(3), p. 288-324 (2000).

[Kol] V.N. Kolokoltsov. Semi-classical analysis for diffusions and stochastic processes. Lecture Notes in Mathematics 1724. Springer Verlag, Berlin 2000.

[KolMa] V.N. Kolokoltsov and K. Makarov. Asymptotic spectral analysis of a small diffusion operator and the life times of the corresponding diffusion process. Russian J. Math. Phys. 4 (3), p. 341-36 (1996).

[Kon] V.A. Kondratiev. Boundary value problems for elliptic equations in domains with conical or angular points. Trudy Moskov. Mat. Obshch. 16, p. 209-292 (1967).

[Ma] S. Maigrot. Encadrement de la première valeur propre d'un opérateur de Schrödinger dégénéré. Preprint 99.06, Université de Reims (1999). 
[Mic] L. Miclo. Comportement de spectres d'opérateurs à basse température. Bull. Sci. Math. 119, p. 529-533 (1995).

[Mil] J.W. Milnor. Lectures on the h-cobordism Theorem. Princeton University press (1965).

[Pe] A. Persson. Bounds for the discrete part of the spectrum of a semibounded Schrödinger operator. Math. Scandinavica 8, p. 143-153 (1960).

[Schw] G. Schwarz. Hodge decomposition. A method for Solving Boundary Value Problems. Lect. Notes in Mathematics 1607, Springer (1995).

[Sima] C.G. Simader. Essential self-adjointness of Schrödinger operators bounded from below. Math. Z. 159, p. 47-50 (1978).

[Sim1] B. Simon. Trace ideals and their applications. Cambridge University Press IX, Lecture Notes Series 35 (1979).

[Sim2] B. Simon. Semi-classical analysis of low lying eigenvalues, I.. Nondegenerate minima: Asymptotic expansions. Ann. Inst. H. Poincaré Phys. Théor. 38, p. 296-307 (1983).

[Wit] E. Witten. Supersymmetry and Morse inequalities. J. Diff. Geom. 17, p. 661-692 (1982).

[Zh] Weiping Zhang. Lectures on Chern-Weil theory and Witten deformations. Nankai Tracts in Mathematics. Vol. 4. World Scientific (2002).

\section{A An example in dimension 1}

We present more directly the 1-dimensional case. We just look at the case of an interval $[a, b]$. We describe techniques which were first developed for Neumann in $[\mathrm{BoHe}]$, adapting to one dimensional problem the techniques developed in [HelSj1]. We just take the simple example of an interval $(a, b)$ with $a<0<b$ and the Dirichlet realization of the semi-classical Witten Laplacian

$$
\Delta_{f, h}^{(0)}:=-h^{2} \frac{d^{2}}{d x^{2}}+f^{\prime}(x)^{2}-h f^{\prime \prime}(x)
$$

associated to a function $f$ on $C^{\infty}([a, b])$ admitting a unique minimum at 0

$$
f(0)=f^{\prime}(0)=0
$$


and no local maxima :

$$
f^{\prime}(x) \neq 0 \text { on }[a, b] \backslash\{0\} .
$$

In particular we get :

$$
f^{\prime}(a)<0, f^{\prime}(b)>0 \text {. }
$$

The function $(a, b) \ni x \mapsto u_{h}:=\exp -\frac{f(x)}{h}$ satisfies

$$
\Delta_{f, h}^{(0)} \exp -\frac{f((x)}{h}=0
$$

but does not satisfy the Dirichlet condition at $a$ and $b$. Of course, one can take a cut-off function $\chi$ with compact support in $(a, b)$ and equal to one on $[a+\epsilon, b-\epsilon)$ but considering $u_{\chi}=\chi u_{h}$, we get

$$
\Delta_{f, h}^{(0)}\left(\chi u_{h}\right)=\mathcal{O}\left(\exp -\frac{\min (f(a), f(b))}{h}\right) \exp \frac{\theta(\epsilon)}{h}
$$

with $\theta(\epsilon) \rightarrow 0$ as $\epsilon \rightarrow 0$.

The best which can be obtained with this construction is the following estimate for the ground state energy :

$$
0 \leq \lambda_{1}(h) \leq C_{\eta}\left(\exp -\frac{2 \min (f(a), f(b))}{h}\right) \exp \frac{\eta}{h}, \forall \eta>0 .
$$

By taking an $\eta$-dependent cut-off function $(\eta=C h \log h)$, one can arrive to

$$
\lambda_{1}(h)=h^{-N} \mathcal{O}\left(\exp -\frac{2 \min (f(a), f(b))}{h}\right),
$$

for some $N>0$.

This does not give a lower bound. We also observe that this quasimode works also for the Neumann problem.

In order to have a better result, one can simply proceed in the following way. Let us assume for simplification that

$$
f(a)<f(b)
$$

Then the main effect is in $a$ and we can continue to use a simple cut-off near $b$. In order to satisfy the Dirichlet condition at $a$, we have to add a correction. For this we need another "formal" solution, which is given by the 


\section{Lemma A.1.}

For any formal series $\sum_{j} \alpha_{j} h^{j}$, there exists on $\left[a, a+\eta_{0}\right)\left(\eta_{0}>0\right)$ a formal WKB solution

$$
u_{-}^{w k b}:=c(x, h) \exp \frac{f(x)}{h},
$$

in the kernel of $\Delta_{f, h}^{(0)}$, such that

$$
c(x, h) \sim \sum_{j \geq 0} c_{j}(x) h^{j}
$$

and

$$
c(a, h) \sim \sum_{j} \alpha_{j} h^{j}
$$

\section{Proof}

We expand the relation :

$$
\exp -\frac{f(x)}{h} \Delta_{f, h}^{(0)}\left(c(x, h) \exp \frac{f(x)}{h}\right) \sim 0
$$

in powers of $h$.

This explicitely leads to the following equation :

$$
2 f^{\prime \prime} c+2 f^{\prime} c^{\prime}+h c^{\prime \prime} \sim 0
$$

or

$$
\left[2 c f^{\prime}+h c^{\prime}\right]^{\prime} \sim 0 .
$$

We first observe that the coefficient of $h^{0}$ vanishes (this corresponds to the fact that $-f$ is a solution of the eikonal equation). Looking now at the coefficient of $h$, we obtain :

$$
-2 f^{\prime}(x) c_{0}^{\prime}(x)-2 f^{\prime \prime}(x) c_{0}(x)=0, c_{0}(a)=\alpha_{0} .
$$

Observing that $f^{\prime}(x) \neq 0$ near $a$, there is no problem for solving the equation, in the neighborhood of $a$, which can be more simply written as

$$
\left(c_{0} f^{\prime}\right)^{\prime}=0, c_{0}(a)=\alpha_{0} .
$$

At the step $j+1$, we will find :

$$
-2 f^{\prime}(x) c_{j}^{\prime}(x)-2 f^{\prime \prime}(x) c_{j}(x)=c_{j-1}^{\prime \prime}(x), c_{j}(a)=\alpha_{j},
$$

or

$$
2 c_{j}(x) f^{\prime}(x)+c_{j-1}^{\prime}(x)=2 \alpha_{j} f^{\prime}(a)+c_{j-1}^{\prime}(a) .
$$




\section{The good quasimode}

We define :

$$
u^{w k b}=\chi u_{h}-\exp -\frac{2 f(a)}{h} \tilde{\chi} u_{-}^{w k b}
$$

where

- $\chi$ satisfies $\chi=1$ on $[a, b-\epsilon)$ and vanishes near $b$;

- $u_{+}^{w k b}$ is associated to $\alpha_{0}=1, \alpha_{j}=0$ for $j>0$;

- $\tilde{\chi}$ satisfies $\tilde{\chi}=1$ on $\left[a, a+\epsilon_{0}\right)$ and vanishes outside $\left[a, a+2 \epsilon_{0}\right)$.

Here $\epsilon$ and $\epsilon_{0}$ can be chosen arbirarily small (one condition is $2 \epsilon_{0}<\eta_{0}$ ) but will be then fixed independently of $h$.

We fix some summation (by the Borel procedure) for $c(x, h)$ with the property that $c(a, h)=1$. So the corresponding function $u^{w k b}$ (we use the same notation) satisfies the Dirichlet condition at $a$ and $b$. Let us compute :

$$
\begin{aligned}
\Delta_{f, h}^{(0)} u^{w k b}= & {\left[\Delta_{f, h}^{(0)}, \chi\right] u_{h} } \\
& -\exp -\frac{2 f(a)}{h}\left[\Delta_{f, h}^{(0)}, \tilde{\chi}\right] u_{-}^{w k b} \\
& -\exp -\frac{2 f(a)}{h} \tilde{\chi} \Delta_{f, h}^{(0)} u_{-}^{w k b} .
\end{aligned}
$$

There are three terms in the right hand side that we write $r_{1}+r_{2}+r_{3}$ and that we analyze separately.

- $r_{1}$ is supported near $b$ and its size is (with in mind our assumption that $f(a)<f(b))$ of order $\mathcal{O}\left(\exp -\frac{f(b)}{h}\right) \exp \frac{\theta(\epsilon)}{h}$. We can choose $\epsilon>0$ such that:

$$
\left\|r_{1}\right\|_{L^{2}}=\mathcal{O}\left(\exp -\frac{f(a)}{h}\right) \exp -\frac{\eta_{1}}{h}, \quad \operatorname{supp} r_{1} \subset(b-\epsilon, b),
$$

for some $\eta_{1}>0$.

- $r_{2}$ is supported in $\left(a+\epsilon_{0}, a+2 \epsilon_{0}\right)$ and its size is $\exp -\frac{2 f(a)}{h} \exp \frac{f\left(a+\epsilon_{0}\right)}{h}$. If we observe that $f\left(a+\epsilon_{0}\right)<f(a)$, we get

$$
\left\|r_{2}\right\|_{L^{2}}=\mathcal{O}\left(\exp -\frac{f(a)}{h}\right) \exp -\frac{\eta_{2}}{h}, \quad \operatorname{supp} r_{2} \subset\left(a+\epsilon_{0}, a+2 \epsilon_{0}\right),
$$

for some $\eta_{2}>0$. 
- $r_{3}$ is supported in $\left[a, a+2 \epsilon_{0}\right)$ and its size is $\mathcal{O}\left(h^{\infty}\right) \exp -\frac{2 f(a)}{h} \exp \frac{f(x)}{h}$. In particular, we get :

$$
\left\|r_{3}\right\|_{L^{2}}=\mathcal{O}\left(h^{\infty}\right) \exp -\frac{f(a)}{h}, \quad \operatorname{supp} r_{3} \subset\left[a, a+2 \epsilon_{0}\right) .
$$

So this is $r_{3}$ which is the dominant term for the computation of the $L^{2}$ norm of the error and we have finally obtained

$$
\Delta_{f, h}^{(0)} u^{w k b}=\mathcal{O}\left(h^{\infty}\right) \exp -\frac{f(a)}{h}
$$

in $L^{2}((a, b))$, for a suitable choice of $\epsilon$ and $\epsilon_{0}$.

It is easy, to get a lower bound for $\left\|u^{w k b}\right\|$ assuming for example

$$
f^{\prime \prime}(0)>0 \text {. }
$$

In this case, we immediately get from this first computation, that there is a unique eigenvalue of $\Delta_{f, h}^{(0), D i r}$ in the interval $\left[0, h^{\frac{3}{2}}\right]$ which is actually exponentially small and that there exists $\rho(h) \sim h^{-\frac{1}{4}} \rho_{0}$ with $\rho_{0} \neq 0$ such that the normalized positive eigenvector $v_{1}(x, h)$ satisfies :

$$
v_{1}(x, h)-\rho(h) u^{w k b}=\mathcal{O}\left(h^{\infty}\right) \exp -\frac{f(a)}{h} .
$$

We note also that $h^{\frac{1}{4}} \rho(h)$ has a complete expansion in powers of $h$, depending only on the Taylor expansion of $f$ at the origin. We have indeed :

$$
\frac{1}{\rho(h)^{2}} \sim\left\|u^{w k b}\right\|^{2}
$$

In this situation, elementary Hilbertian computations (see [HelSj1]) give that:

$$
\lambda_{1}(h)=\frac{\left\langle\Delta_{f, h}^{(0)} u^{w k b} \mid u^{w k b}\right\rangle}{\left\|u^{w k b}\right\|^{2}}+\mathcal{O}\left(h^{\infty}\right) \exp -\frac{2 f(a)}{h} .
$$

For a more precise estimate of the right hand side, we have consequently to come back to a more careful estimation of the terms $\left\langle r_{j} \mid u^{w k b}\right\rangle$ modulo $\mathcal{O}\left(h^{\infty}\right) \exp -\frac{2 f(a)}{h}$. Let us determine the significant terms.

- We can clearly forget $\left\langle r_{1} \mid u^{w k b}\right\rangle$ which satisfies, for $\eta_{1}>0$,

$$
\exp \frac{2 f(a)}{h}\left\langle r_{1} \mid u^{w k b}\right\rangle=\exp -\frac{\eta_{1}}{h} \text {. }
$$


- For $r_{2}$, we get :

$$
\exp \frac{2 f(a)}{h}\left\langle r_{2} \mid u^{w k b}\right\rangle=\exp \frac{2 f(a)}{h}\left\langle r_{2} \mid u_{h}\right\rangle+\mathcal{O}\left(h^{\infty}\right) .
$$

- For $r_{3}$, we get :

$$
\exp \frac{2 f(a)}{h}\left\langle r_{3} \mid u^{w k b}\right\rangle=\mathcal{O}\left(h^{\infty}\right) .
$$

From this analysis, we get :

$$
\lambda_{1}(h)=\frac{\left\langle r_{2} \mid u_{h}\right\rangle}{\left\|u_{h}\right\|^{2}}+\mathcal{O}\left(h^{\infty}\right) \exp -\frac{2 f(a)}{h},
$$

with $r_{2}$ defined after (A.20). So

$$
\exp \frac{2 f(a)}{h} \lambda_{1}(h)=\frac{\left\langle\left[\Delta_{f, h}^{(0)}, \tilde{\chi}\right] u_{-}^{w k b} \mid u_{h}\right\rangle}{\left\|u_{h}\right\|^{2}}+\mathcal{O}\left(h^{\infty}\right) .
$$

The computation is now elementary (and rather standard).

$$
\begin{aligned}
\left\langle\left[\Delta_{f, h}^{(0)}, \tilde{\chi}\right] u_{-}^{w k b} \mid u_{h}\right\rangle & =-h \int_{a}^{b}\left(h\left(\tilde{\chi}^{\prime \prime} c(x, h)+2 \tilde{\chi}^{\prime} c^{\prime}\right)+2 \tilde{\chi}^{\prime} c(x, h)\right) f^{\prime}(x) d x \\
& =-h \int_{a}^{b} \tilde{\chi}^{\prime}\left[2 c f^{\prime}+c^{\prime}\right] d x \\
& \sim-2 h f^{\prime}(a)-h^{2} c^{\prime}(a) .
\end{aligned}
$$

In the last line, we have used the eikonal equation (modulo $\mathcal{O}\left(h^{\infty}\right)$ ) and an integration by parts. We are happy to recover as expected that the result is independent of the choice of $\tilde{\chi}$, with the above properties. We finally get :

$$
\exp \frac{2 f(a)}{h} \lambda_{1}(h)=\frac{d(h)}{\left\|u_{h}\right\|^{2}}+\mathcal{O}\left(h^{\infty}\right),
$$

with

$$
d(h)=-2 h f^{\prime}(a)(1+\mathcal{O}(h)) .
$$

So we have proved :

\section{Proposition A.2.}

Under assumptions (A.2), (A.3), (A.4), (A.8) and (A.25), the lowest eigenvalue of $\Delta_{h, f}^{(0)}$ has the following expansion:

$$
\exp \frac{2 f(a)}{h} \lambda_{1}(h)=-2(\pi)^{-\frac{1}{2}} h^{\frac{1}{2}} f^{\prime}(a) f^{\prime \prime}(0)^{\frac{1}{2}}(1+\mathcal{O}(h)) .
$$


Note that there are in principle no problem for computing explicitly a complete expansion of the right hand side in (A.36). Note also that we have proceeded differently in the general case but that we of course recover (A.36) as subcase of Theorem 1.1.

\section{Remark A.3.}

The treatment in our main text is a little different but we recall that by applying $d_{f, h}$ to the localized quasimode constructed for $\Delta_{f, h}^{(0)}$ near a or near $b$, we get two orthogonal quasimodes showing the existence of a spectral space of dimension $\geq 2$ corresponding to exponentially small eigenvalues. 